

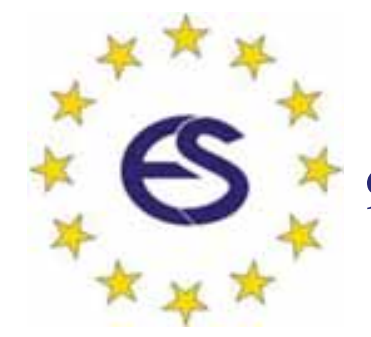

\section{EUROSIM 2019 \\ $9^{\text {th }}$ EUROSIM Congress on Modelling and Simulation \\ La Rioja, Logroño, Spain, July 2019}
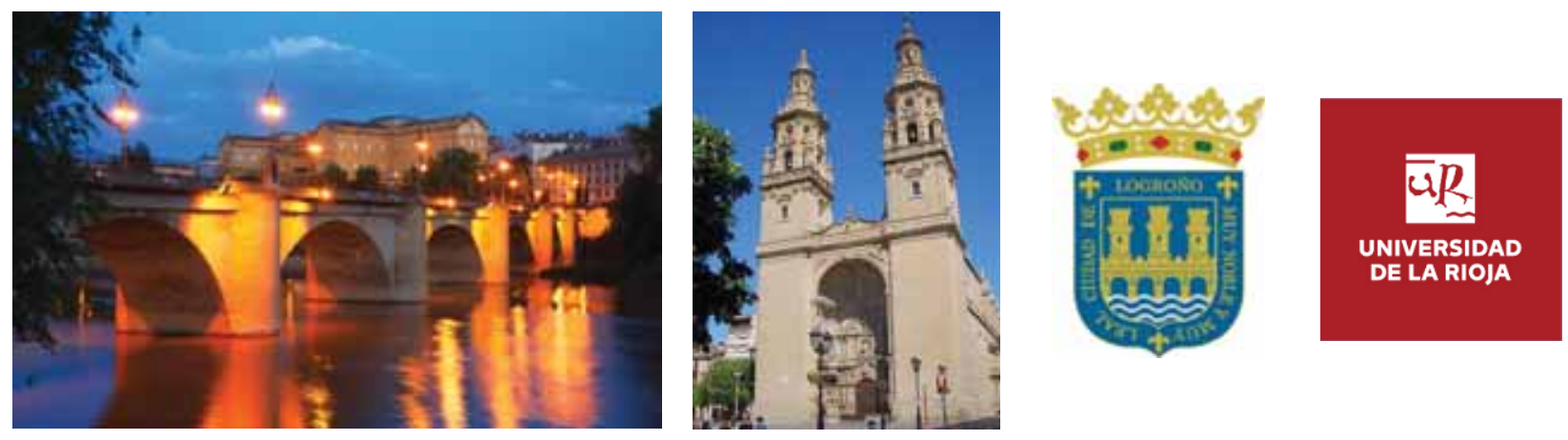

EUROSIM Congresses are the most important modelling and simulation events in Europe. For EUROSIM 2019, we are soliciting original submissions describing novel research and developments in the following (and related) areas of interest: Continuous, discrete (event) and hybrid modelling, simulation, identification and optimization approaches. Two basic contribution motivations are expected: M\&S Methods and Technologies and M\&S Applications. Contributions from both technical and non-technical areas are welcome.

Congress Topics The EUROSIM 2019 Congress will include invited talks, parallel, special and poster sessions, exhibition and versatile technical and social tours. The Congress topics of interest include, but are not limited to:

Intelligent Systems and Applications

Hybrid and Soft Computing

Data \& Semantic Mining

Neural Networks, Fuzzy Systems \&

Evolutionary Computation

Image, Speech \& Signal Processing

Systems Intelligence and

Intelligence Systems

Autonomous Systems

Energy and Power Systems

Mining and Metal Industry

Forest Industry

Buildings and Construction

Communication Systems

Circuits, Sensors and Devices

Security Modelling and Simulation
Bioinformatics, Medicine, Pharmacy and Bioengineering

Water and Wastewater Treatment,

Sludge Management and Biogas Production

Condition monitoring, Mechatronics and maintenance

Automotive applications

e-Science and e-Systems

Industry, Business, Management, Human Factors and Social Issues

Virtual Reality, Visualization, Computer Art and Games

Internet Modelling, Semantic Web and Ontologies

Computational Finance \& Economics
Simulation Methodologies and Tools

Parallel and Distributed

Architectures and Systems

Operations Research

Discrete Event Systems

Manufacturing and Workflows

Adaptive Dynamic Programming and Reinforcement Learning

Mobile/Ad hoc wireless networks, mobicast, sensor placement, target tracking

Control of Intelligent Systems

Robotics, Cybernetics, Control Engineering, \& Manufacturing

Transport, Logistics, Harbour, Shipping and Marine Simulation

Congress Venue / Social Events The Congress will be held in the City of Logroño, Capital of La Rioja, Northern Spain. The main venue and the exhibition site is the University of La Rioja (UR), located on a modern campus in Logroño, capital of La Rioja, where 7500 students are registered. The UR is the only University in this small, quiet region in Northern Spain. La Rioja is where the Monasteries of San Millán de la Cogolla, cradle of the first words written in the Spanish language, are situated, sites included in UNESCO's World Heritage List in 1996. Of course, social events will reflect this heritage - and the famous wines in la Rioja.

Congress Team: The Congress is organised by CAE CAE-SMSG, the Spanish simulation society, and Universidad de la Rioja.

Info: Emilio Jiménez, EUROSIM President, emilio.jimenez@unirioja.es Juan Ignacio Latorre, juanignacio.latorre@unavarra.es 


\section{Editorial}

Dear Readers - SNE Volume 27 (2017) provides novelties in content and organisation. SNE has updated and sharpened aims and scope-discussed in the editorial of the last two issues. This issue SNE 27(3), underlines SNE's publication strategy for EUROSIM societies: SNE is open for post-conference publication of contributions from EUROSIM conferences - as with this issue.

SNE is the official membership journal of EUROSIM, the Federation of European simulation societies and simulation groups. Members of EUROSIM societies, as ASIM (the German simulation society), or CEA-SMSG, the Spanish IFAC-based simulation society, and seventeen other societies) have privileged access to online SNE contributions: society login and society password allow download of colour high-resolution publications of scientific contributions, indicated in the picture at right with file name appendix ' $R A$ '. The snapshot at right refers to the new SNE website www.sne-journal.org, which together with the new EUROSIM website www.eurosim.info offers benefits for EUROSIM members.

\section{SNE SIMULATION}

Simulation Notes Europe, Volume 27(1), March 2017

A Model Factory in Augmented Reality as an Eye-catcher at Exhibitions and Fairs

Torsten Munkelt | Steven Behne | Markus Wacker | Sven volker

Simulation Notes Furope SNE 27(1), 2017, 1-8

DOI: $10.11128 /$ sne.27.tn.10361

Abstract

Our universities required an eye-catcher at exhibitions, conferences, open houses etc. We decided in $f$

Related Files

- sne.27.1.10361.tn.OA.pdf | $301 \mathrm{~KB}$

- sne.27.1.10361.tn.RA.pdf | 939 KB

I would like to thank all authors for their contributions to SNE 27(3) showing the broad variety of simulation. And thanks to the editorial board members for review and support, and to the organizers of the EUROSIM conferences for co-operation in postconference contributions. And last but not least thanks to the SNE Editorial Office for layout, typesetting, preparations for printing, with special thanks for support in relaunch of SNE website.

Felix Breitenecker, SNE Editor-in-Chief,eic@sne-journal.org; felix.breitenecker@tuwien.ac.at

\section{Contents SNE 27(3)}

Online SNE 27(3), DOI 10.11128/ sne.27.3.1038, ARGESIM Publ.

Print SNE 27(3) ISBN 978-3-903024-61-8,

Print-on-Demand, TU Verlag Vienna, www.tuverlag.at

A New Approach for Integrating Discrete Element

Method into Component-Oriented System Simulations

C. Richter

Design of Simulation Model for Novel Solar

Thermal Storage Tank.

M. Narayanan, G. Mengedoht, W. Commerell

Conceptional Problems of Transaction-based Modeling and its Implementation in SimEvents 4.4.

L. Austermann, P. Junglas, J. Schmidt, C. Tiekmann

Automatic Layout of Scilab/Xcos Diagrams.

C. Zhu, U. Durak, S. Hartmann, C. David

Mathematical Wave Fitting Models for the Quantification of the Diurnal Prolle and Variability of Pulse Wave Analysis Parameters.

L. Bauer, B. Hametner, C. Mayer, S. Wassertheurer

Physical Brain Connectivity Models for Functional

Magnetic Resonance Imaging,

N. Geissberger, G. Schneckenreither

EUROSIM Societies Short Info $\mathrm{N} 1-\mathrm{N} 8$

\section{SNE Contact \& Info}

$\rightarrow$ www.sne-journal.org

玤 office@sne-journal.org, eic@sne-journal.org

$\triangle$ SNE Editorial Office, Andreas Körner ARGESIM / M ath. M odelling \& Simulation Group, Vienna Univ. of Technology / 101, Wiedner Haupstrasse 8-10, 1040 Vienna , Austria

\section{SNE Simulation Notes EURope}

\section{SNE Online ISSN 2306-0271, SNE Print ISSN 2305-9974}

WEB: $\rightarrow$ www.sne-journal.org, DOI prefix 10.11128/sne

Scope: Developments and trends in modelling and simulation in various areas and in application and theory; comparative studies and benchmarks (documentation of ARGESIM Benchmarks on modelling approaches and simulation implementations); modelling and simulation in and for education, simulation-based e-learning; society information and membership information for EUROSIM members (Federation of European Simulation Societies and Groups).

Editor-in-Chief: Felix Breitenecker, Vienna Univ. of Technology, Math. Modelling and Simulation Group

竹Felix.Breitenecker@tuwien.ac.at, 㭋 eic@sne-journal.org

Layout / Administration: A. Körner, A. Mathe, J. Tanzler, R. Leskovar, et al.; $\equiv=$ office@sne-journal.org

Print SNE and Print-on-Demand: Grafisches Zentrum and TU-Verlag, TU Wien, Wiedner Hauptstrasse 8-10, 1040, Vienna, Austria www.tuverlag.at

Publisher: ARGESIM ARbeITSGem EINSChaft Sim ULATION NeWS C/O M ath. Modelling and Simulation Group, TU Wien/101, Wiedner Hauptstrasse 8-10, 1040 Vienna, Austria; www.argesim.org, ASIM www.asim-gi.org and EUROSIM $\rightarrow$ www.eurosim.info (c) ARGESIM / EUROSIM / ASIM 2017 


\section{SNE - Aims and Scope}

Simulation Notes Europe (SNE) provides an international, high-quality forum for presentation of new ideas and approaches in simulation - from modelling to experiment analysis, from implementation to verification, from validation to identification, from numerics to visualisation - in context of the simulation process.

SNE seeks to serve scientists, researchers, developers and users of the simulation process across a variety of theoretical and applied fields in pursuit of novel ideas in simulation and to enable the exchange of experience and knowledge through descriptions of specific applications. SNE follows the recent developments and trends of modelling and simulation in new and/or joining application areas, as complex systems and big data. SNE puts special emphasis on the overall view in simulation, and on comparative investigations, as benchmarks and comparisons in methodology and application. For this purpose, SNE documents the ARGESIM Benchmarks on Modelling Approaches and Simulation Implementations with publication of definitions, solutions and discussions. SNE welcomes also contributions in education in/for/with simulation.

A News Section in SNE provides information for EUROSIM Simulation Societies and Simulation Groups.

SNE, primarily an electronic journal, follows an open access strategy, with free download in basic layout. SNE is the official membership journal of EUROSIM, the Federation of European Simulation Societies and Simulation Groups www.eurosim.info. Members of EUROSIM societies are entitled to download SNE in an elaborate and extended layout, and to access additional sources of benchmark publications, model sources, etc. Print SNE is available for specific groups of EUROSIM societies, and starting with Volume 27 (2017) as printon-demand from TU Verlag, TU Wien. SNE is DOI indexed by CrossRef, identified by DOI prefix 10.11128, assigned to the SNE publisher ARGESIM (www.argesim.org).

Author's Info. Individual submissions of scientific papers are welcome, as well as post-conference publications of contributions from conferences of EUROSIM societies. SNE welcomes special issues, either dedicated to special areas and/or new developments, or on occasion of events as conferences and workshops with special emphasis.

Authors are invited to submit contributions which have not been published and have not being considered for publication elsewhere to the SNE Editorial Office.

SNE distinguishes different types of contributions (Notes), i.e.

- TN Technical Note, 6-10 p. - EN Education Note -6-8 p.

- PN Project Note 6-8 p. - SN Short Note, max. 6 p.

- SW Software Note, 4-6 p. - BN Benchmark Note, 4-8 p.

- ON Overview Note $O N$ - only upon invitation, up to $14 \mathrm{p}$.

Further info and templates (doc, tex) at SNE‘s website. www.sne-journal.org

\section{SNE Editorial Board}

SNE - Simulation Notes Europe is advised and supervised by an international scientific editorial board. This board is taking care on peer reviewing of submission to SNE. At present, the board is increasing (see website www.sne-journal.org):

David Al-Dabass, david.al-dabass@ntu.ac.uk, Nottingham Trent University, UK

Felix Breitenecker, Felix.Breitenecker@tuwien.ac.at Vienna Univ. of Technology, Austria, Editor-in-chief

Maja Atanasijevic-Kunc,maja.atanasijevic@fe.uni-lj.si Univ. of Ljubljana, Lab. Modelling \& Control, Slovenia

Aleš Belič, ales.belic@sandoz.com Sandoz / National Inst. f. Chemistry, Slovenia

Peter Breedveld, P.C.Breedveld@el.utwente.nl University of Twenty, Netherlands

Agostino Bruzzone,agostino@itim.unige.it Universita degli Studi di Genova, Italy

Francois Cellier,fcellier@inf.ethz.ch ETH Zurich, Switzerland

Vlatko Čerić,vceric@efzg.hr Univ. Zagreb, Croatia

Russell Cheng,rchc@maths.soton.ac.uk University of Southampton, UK

Roberto Cianci,cianci@dime.unige.it, Math. Eng. and Simulation, Univ. Genova, Italy

Eric Dahlquist,erik.dahlquist@mdh.se, Mälardalen Univ., Sweden

Horst Ecker,Horst.Ecker@tuwien.ac.at Vienna Univ. of Technology, Inst. f. Mechanics, Austria

Vadim Engelson, vadim.engelson@mathcore.com MathCore Engineering, Linköping, Sweden

Edmond Hajrizi, ehajrizi@ubt-uni.net University for Business and Technology, Pristina, Kosovo

András Jávor, javor@eik.bme.hu, Budapest Univ. of Technology and Economics, Hungary

EskoJuuso,esko.juuso@oulu.fi Univ. Oulu, Dept. Process/Environmental Eng., Finland

Kaj Juslin, kaj.juslin@vtt.fi VTT Technical Research Centre of Finland, Finland

Andreas Körner, andreas.koerner@tuwien.ac.at Technical Univ. Vienna, E-Learning Dpt., Vienna, Austria

Francesco Longo,f.longo@unical.it Univ. of Calabria, Mechanical Department, Italy

Yuri Merkuryev, merkur@itl.rtu.lv, Riga Technical Univ.

David Murray-Smith,d.murray-smith@elec.gla.ac.uk University of Glasgow, Fac. Electrical Engineering, UK

Gasper Music,gasper.music@fe.uni-lj.si Univ. of Ljubljana, Fac. Electrical Engineering, Slovenia

Thorsten Pawletta,pawel@mb.hs-wismar.de Univ. Wismar, Dept. Comp. Engineering, Wismar, Germany

Niki Popper,niki.popper@dwh.at dwh Simulation Services, Vienna, Austria

Kozeta Sevrani, kozeta.sevrani@unitir.edu.al Univ. Tirana, Inst.f. Statistics, Albania

Thomas Schriber,schriber@umich.edu University of Michigan, Business School, USA

Yuri Senichenkov, sneyb@dcn.infos.ru St. Petersburg Technical University, Russia

Oliver Ullrich, oullrich@cs.1u.edu Florida International University, USA

Siegfried Wassertheurer, Siegfried.Wassertheurer@ait.ac.at AIT Austrian Inst. of Technology, Vienna, Austria

Sigrid Wenzel, S.Wenzel@uni-kassel.de Univ. Kassel, Inst. f. Production Technique, Germany 


\title{
A New Approach for Integrating Discrete Element Method into Component-oriented System Simulations
}

\author{
Christian Richter \\ Technische Universität Dresden, Chair of Construction Machinery; christian.richter1@tu-dresden.de
}

SNE 27(3), 2017, 125 - 130, DOI: 10.11128/sne.27.tn.10381 Received: June 1, 2017 (Selected ASIM GMMS/STS 2017 Postconf. Publ.), Accepted: July 20, 2017

SNE - Simulation Notes Europe, ARGESIM Publisher Vienna, ISSN Print 2305-9974, Online 2306-0271, www.sne-journal.org

\begin{abstract}
The working process of construction and conveying machines is characterized by the interaction with granular and bulk materials. In order to allow prospective analysis of machine behaviour under real operating conditions, coupled simulations are increasingly used. While modelling the equipment happens within the scope of component-oriented system modelling, reproducing particle-mechanical behaviour is done with discrete element method. The work presented here introduces a new integrated approach which allows a closed modelling and simulation of system models and discrete element method. The creation and calculation of coupled simulations is thus facilitated by a multiple.
\end{abstract}

\section{Introduction}

In recent years the usage and importance of simulations has significantly increased in the field of construction and conveying machines. Both sectors have in common that some kind of machine is handling with some kind of granular material (e.g. sand, gravel, pellets). Typical processes are the digging of a hole with an excavator or the transport of material with a conveyor belt. Knowing the forces arising from these processes is very important. On the one side they cause strains on single parts, on the other side the can affect the entire machine behaviour. Let's consider a excavator for example. The forces coming from the digging process are acting on the bucket and causing stress and wear, but they will also lead to an increasing hydraulic pressure in the cylinders.
This in turn will affect the pump and engine activity. In order to make prospective statements about machine behaviour under real operating conditions it's necessary to simulate the machine as well as the process in common.

For simulating the equipments behaviour, component-oriented system models are often used. These kind of models are describing the machine as a network of components and subsystems which can be part of different domains like hydraulics, electrical or control engineering. One option for simulating bulk materials is using the discrete element method (DEM). With help of this method it's possible to reproduce the motion of granular materials as well as the strains they cause on mechanical parts.

Bringing both simulation techniques together isn't as easy as it seems. In addition to various methods of calculation, the modelling paradigms are also completely different. Arranging coupled simulations is for that very difficult and time consuming. This paper presents a new integrative approach for closed modelling and simulation control.

\section{Basics}

\subsection{Discrete element method}

The discrete element method (DEM) was presented first in 1973 by Cundall and Strack [1]. It's a numerical method for simulating the behaviour and motion of large numbers of discrete, interacting objects. In most cases, as done here, these objects are referred as particles. Basis of the method is the calculation of forces acting between the particles or between a particle and an adjacent surface. The basic calculation cycle should be explained briefly below.

$$
\text { SNE 27(3) - 9/2017 } 125
$$


After insertion every particle has an initial position and velocity. The simulation loop starts with collision detection. In this phase all particle-particle and particlewall contacts are determined. After that the forces and torques acting on every particle must be calculated. These forces result on the one hand from field forces like gravity and on the other hand from the particle deformation as a consequence of collision. For that different force-deformation laws are used. By summing up all single forces and torques, the translational and angular acceleration of each particle can be obtained. The last step is solving the equations of motion. For that the new positions and velocities are resolved by integrating translational and angular acceleration two times (Equations 1 and 2). Figure 1 shows this loop. It is repeated until a predetermined number of iterations is reached.

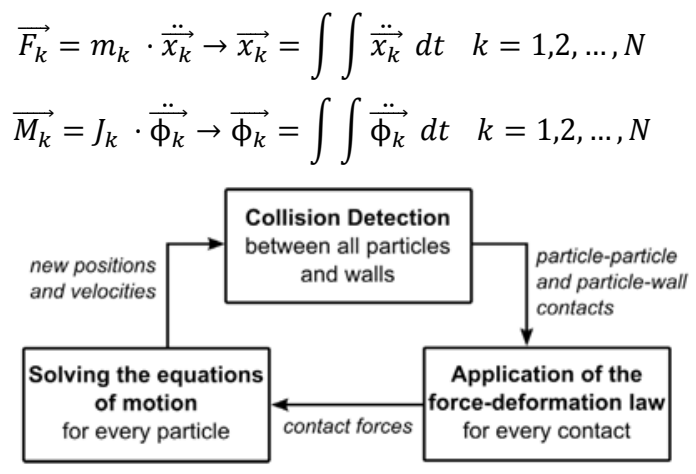

Figure 1: DEM Calculation Loop.

Most software for modelling and simulating discrete element models works command-oriented. The user tells the software what to do by typing single instructions into a command line tool or by loading an input script. Graphical user interfaces are very rare. After typing the commands they are processed sequentially. Typical representatives are open source solutions like LIGGGHTS $^{\circledR}$ and Yade $^{\circledR}$ or commercial tools like PFC3D $^{\circledR}$. This kind of modelling and user-softwareinteraction has historical reasons and is not very user friendly. The biggest problem is that the user has to have good knowledge about the commands and syntax. Furthermore its very complicated to adapt existing models onto new problems. Figure 2 shows an excerpt of a typical input script for LIGGGHTS ${ }^{\circledR}$.

Most of these applications doesn't have any integrated post-processing tool. This is a disadvantage because visualization of results and particle data is very important. For that it's not seldom that users have to use third-party applications like ParaView ${ }^{\circledR}$.

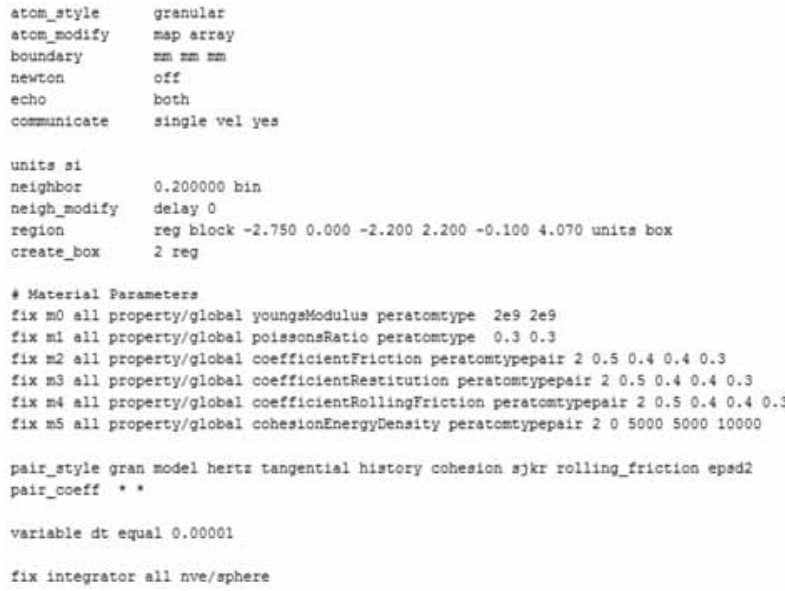

Figure 2: Input Script for LIGGGHTS ${ }^{\circledR}$.

\subsection{Network-based system simulations}

Simulating complex machines - as already mentioned is often done by using equation-based componentoriented system models. For the description of such models Modelica [2] has established as a kind of standard language in many areas. Meanwhile, there is a plurality of applications using and supporting Modelica out there (e.g. Simulation $X^{\circledR}$ or Dymola ${ }^{\circledR}$ ). Modelica allows the model description on several levels. One of the most common types of building up simulation models is the network-based or component-oriented modelling technique. Therefore configurable components and subsystems (network elements), which have clearly defined interfaces, are connected together in a network structure.

Apart from classic advantages of object-oriented approaches, such as encapsulation and re-usability through modularity and inheritance, this method also has other benefits. One is that building up and modifying models can be done very easily and rapidly. A further is the high degree of clarity, as the real and virtual structure often correspond with one another. Exemplary the top-level structure of an engine model is shown in Figure 3.

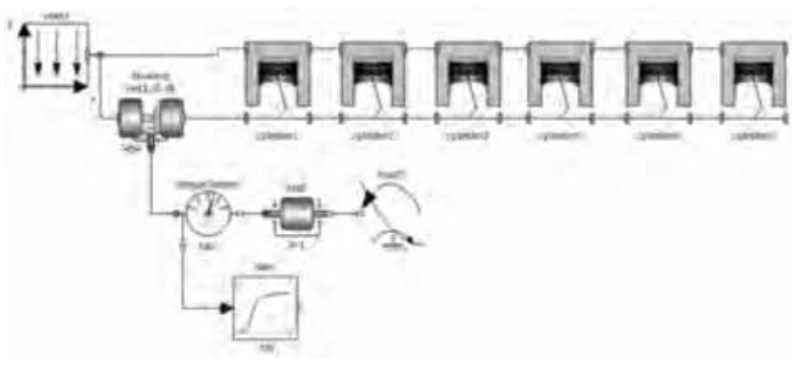

Figure 3: Network model of an engine with 6 cylinders. 


\subsection{Coupled simulations}

As coupled simulation generally the calculation of coupled systems is meant. Coupled-systems consisting of two or more models from different domains, which have to exchange information with each other at simulation runtime. Furthermore the number of integrators and/or modelling tools must be greater than one. According to [3] there are different kinds of coupled simulations with different names as shown in Figure 4.

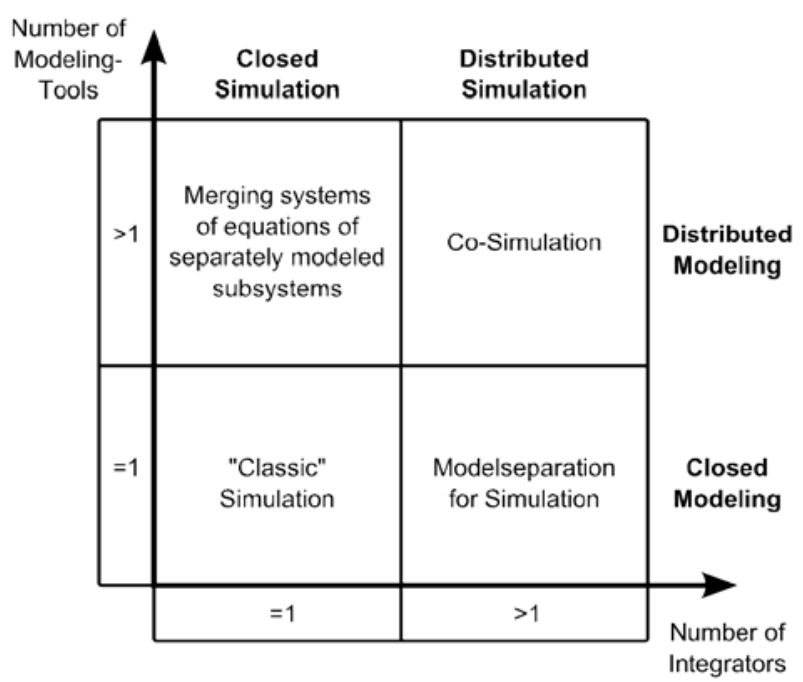

Figure 4: Classification of coupled simulations.

Assuming to this most recent approaches of coupling system simulations with discrete element method must be classified as co-simulation. One example for this is the approach presented in [4]. This approach starts with building up a machine model and exporting it as a functional mock-up unit (FMU). Afterwards a discrete element model is build up and connected to the FMU. The calculation is performed by two integrators with information exchange between both models at discrete time events. Building up and connecting the models causes a lot of work. This amount of work can be reduced by implementing a new functionality which allows closed modelling.

Generally, there are two possibilities for implementing such a functionality. Either it's attempted to integrate the DEM into component-oriented systems modelling or the other way around. From the points and facts mentioned above the first variant seems to be the better way for getting a high degree of usability.

\section{Integrating DEM into Component-oriented System Models}

\subsection{Component library}

For transforming the command-oriented modelling paradigm of classic DEM-tools into a componentoriented modelling technique several things have to be done. First of all, all relevant modelling functions must be identified. After this new abstracted components and parameters must be designed representing and implementing these functions. This components have to be very self-explaining and easy to understand for the user. Table 1 shows a selection of components and their corresponding function.

\begin{tabular}{ll}
\hline Component & Functions \\
\hline SimulationBox & $\begin{array}{l}\text { get total count and mass of all } \\
\text { particles }\end{array}$ \\
\hline ParticleSource & generate particles \\
\hline ParticleSink & remove particles \\
\hline ParticleFlowSensor & $\begin{array}{l}\text { measure the number and mass of } \\
\text { particles passing a surface }\end{array}$ \\
\hline ParticleRegionSensor & $\begin{array}{l}\text { measure the number and and mass } \\
\text { of particles in a volumetric region }\end{array}$ \\
\hline ParticleSet & loading existing data \\
\hline RigidBody & $\begin{array}{l}\text { interaction of a rigid body with the } \\
\text { particles }\end{array}$ \\
\hline
\end{tabular}

Table 1: Components and corresponding functions.

As last step a translator must be implemented, which is capable to translate these components into commando sequences.

\subsection{System structure}

A component library as described before can be used in any Modelica-Tool. It allows the closed modelling of machine and process models. In order to perform a distributed simulation, models have to be subsequently separated. For a better understanding how this is done Figure 5 shows the basic structure of all simulation components. This structure is divided into two main areas - a front-end and a back-end. 


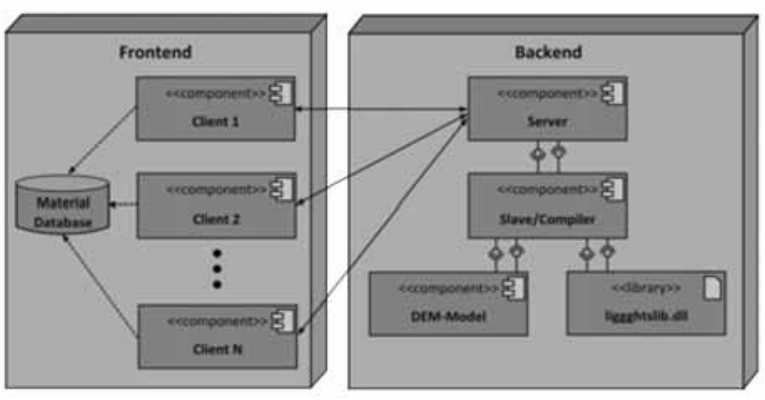

Figure 5: System structure.

The front-end consists essentially of the component library. Each of the components forms a client, which is capable to connect and communicate via TCP/IP to a server. Due to the fact that the presented solution was developed as part of a research project, the component library has some Simulation $\mathrm{X}^{\circledR}$-specific features. One of them is the material selection via a database interface.

The server the components can connect to forms the root node of the back-end-structure. It receives the messages from the components and forwards them to a specific compiler. It's possible that components belonging to different simulation models can connect to the server. For that reason the server can handle more than one compiler at the same time. The compiler uses the information coming from the server to build up a copy of the current component structure. With help of this model commando-sequences are generated which can be executed by a specific DEM-tool. The software used here was LIGGGHTS ${ }^{\circledR}$ compiled as a shared library to make information exchange easier. Holding back the whole component structure at the back-end is necessary for the translation process because some commands can only be created with information coming from two ore more components.

The division into front-end and back-end communicating via TCP with each other seems at first sight a little bit complicated but brings some advantages. One of them is the fact that distributed computations are made possible. That means solving the system model can be done on a normal computer while discrete element simulation runs on a workstation or computer cluster. Especially for time consuming and expensive DEM simulations that's a plus.

\subsection{Communication}

At this point, communication between front-end and back-end is briefly explained. At the beginning of every simulation - that means during initialization phase of the system model - all components respectively clients connect to the server. Required connection settings, such as server address and port, are coming from the so called SimulationBox-component. The following code snippet shows this.

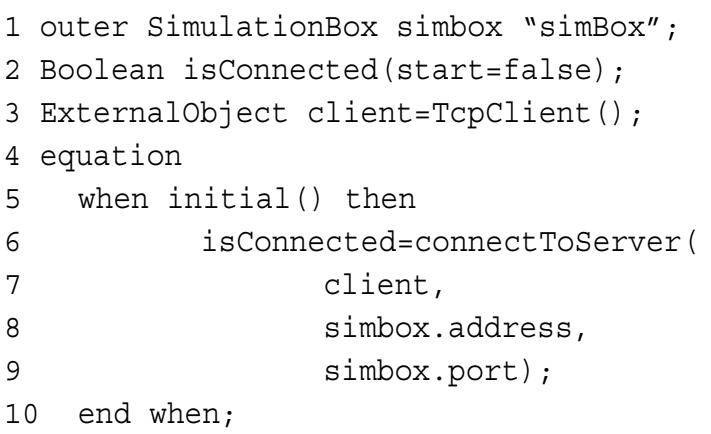

The SimulationBox is a kind of superordinate parent or world object. Besides basic simulation settings it contains information about the spatial domain for DEM and gravity forces acting on all particles. All other components can access this information.

After a successful connection, the communication between front-end and back-end starts. It's divided into three phases:

1. During initial phase all front-end components send their parametric information to the server. These are, for example, initial positions and orientation, geometrical data or material values. All this information are collected at the back-end and used to generate executable commando sequences.

2. At simulation runtime information are exchanged in regular intervals. Here mainly new calculated positions of rigid bodies are transmitted from front- to back-end and forces as well as torques are returned.

3. At the end of simulation an information is sent to the server, which tells this that calculation is over. This will reset the back-end simulation.

Simplified code, containing the complete communication algorithm, is shown below. 
1 parameter Real tc $=0.0001$;

2 Boolean commTrigger $=$ sample $(0$, tc $)$;

3 algorithm

if isconnected then

when initial() then

...send initial data...

elsewhen commTrigger then

....send and receive data...

elsewhen terminal() then

...send final data...

end when;

end if:

\section{Bucket Elevator}

\subsection{Analytical considerations}

For functional testing and evaluating the new solution a bucket elevator was modelled and simulated. A bucket elevator is a mechanism for hauling flow able bulk materials (e.g. grain or sand) vertically. For that it is often used in hoppers.

Of decisive importance in analysing and constructing bucket elevators is material deflation. Basically there are two ways of emptying the bucket at the upper turning point. Either the material is thrown out by centrifugal forces or the material falls out in reaction to gravity forces. The second variant should be avoided because there's a high risk that particles fall back into the elevator housing.

To figure out which kind of material deflation is dominating point $P$ is constructed like shown in Figure 6.

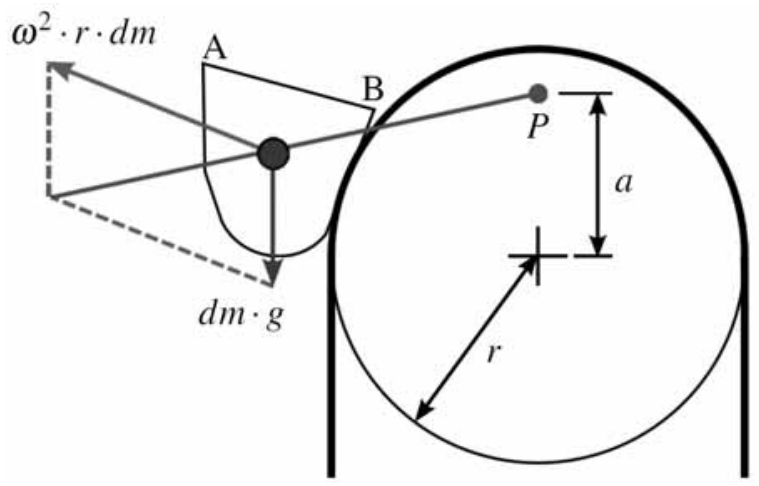

Figure 6: Construction of point $\mathrm{P}$ and distance $\mathrm{a}$.

Thus particles are thrown out over edge $A$ there must be $a<r$ [5]. For achieving this the minimal rotational velocity can be calculated like in Equation 3.

$$
\frac{g}{\omega^{2}}=a ; a<r \rightarrow \omega>\sqrt{\frac{g}{r}}
$$

What is completely ignored in this calculation is the internal friction of the material and the friction between material and bucket wall. So there's no possibility to get reliable prospective statements if the bucket is emptied the right way. Furthermore it's very difficult to investigate the bucket filling process or dynamic forces acting on the drive chain. That's where the discrete element method can help.

\subsection{Modelling}

First step to simulate the bucket elevator was to create a simple machine model, including buckets, housing and a simplified drive train. After this was done it was extended by adding DEM specific components. For continuous filling a particle source was inserted at the lower housing aperture. This particle source has an output rate of 35000 particles every second. At the upper outlet a particle sensor was added for measuring the mass and particle flow leaving the bucket elevator. Additionally another particle sensor was added for measuring the number of particles falling back into the housing. The complete model is shown in Figure 7.

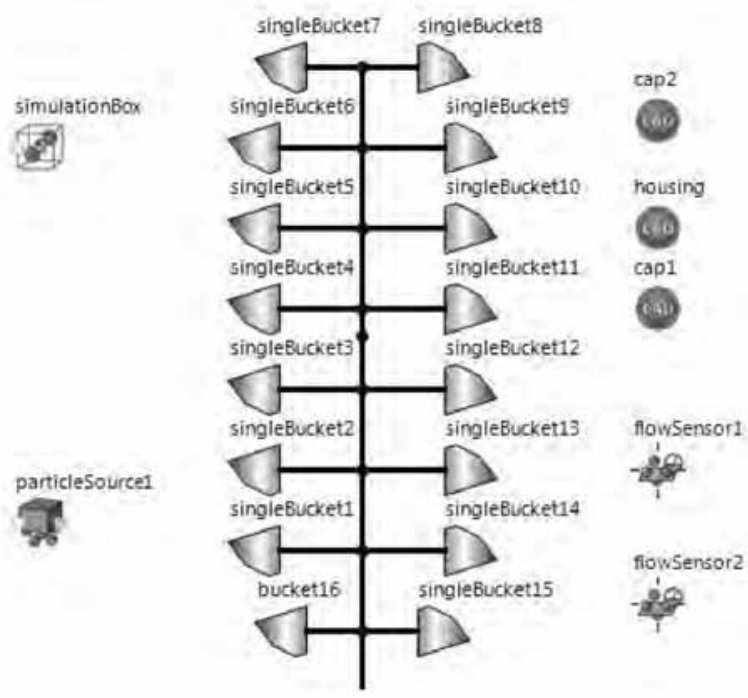

Figure 7: Structure view of the bucket elevator model in Simulation $X^{\circledR}$.

\subsection{Results}

For evaluation the bucket elevator was simulated for 4.0 seconds. After about 3.0 seconds, a steady state is achieved, at which the number of particles inserted is approximately equal to the number of particles leaving the bucket elevator. At this point of time there are about 65000 particles in the system. Figure 8 shows the $3 d-$ simulation-view at this point of time. 


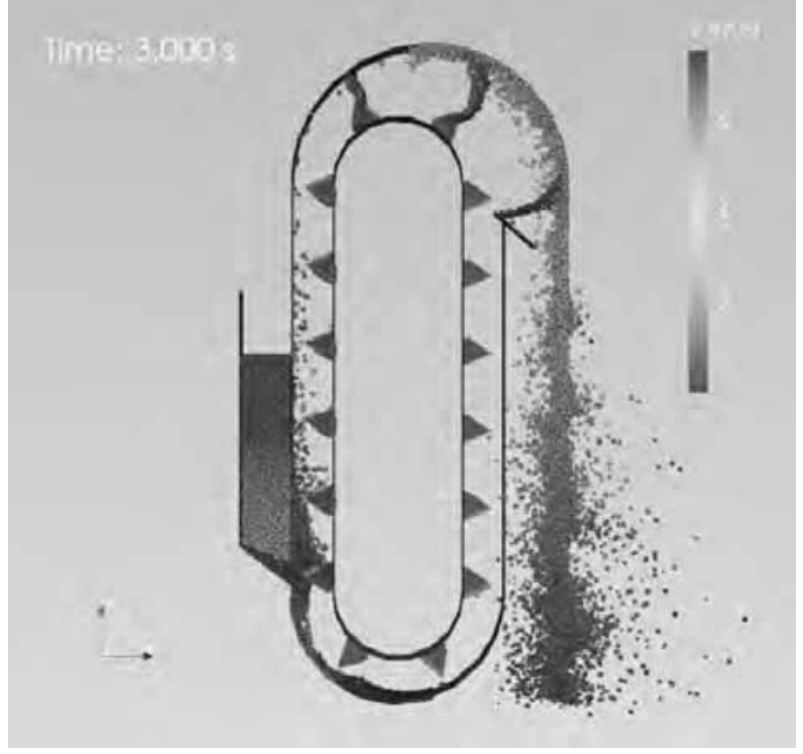

Figure 8: 3d-simulation-view of steady state.

It has been found that at a peripheral speed of $4 \mathrm{~m} / \mathrm{s}$ nearly no particles fall back into the housing. Furthermore the forces acting on all buckets can be measured and used for optimizing the bucket design and drive chain. The temporal course of forces acting on a single bucket is exemplary shown in Figure 9.
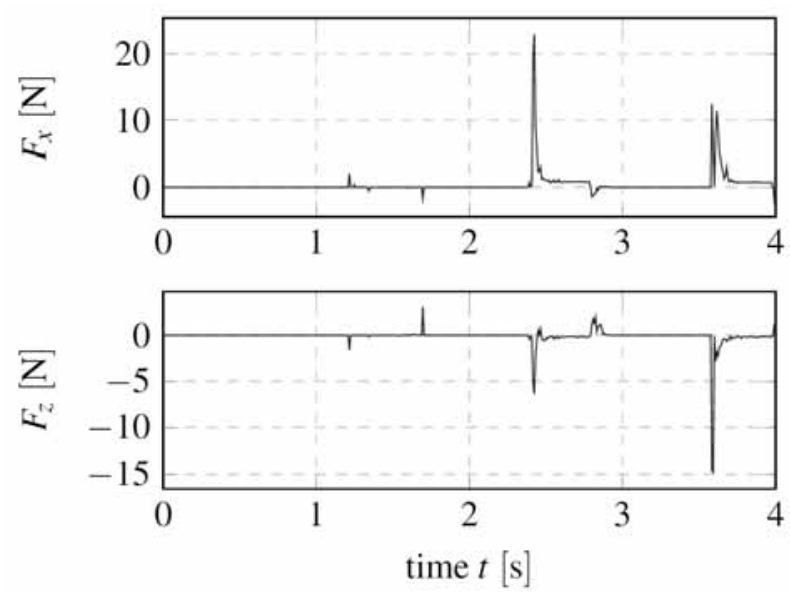

Figure 9: Forces on single bucket.

\section{Conclusion}

In this work, a new concept was presented allowing the closed modelling of machine models and discrete element systems in one simulation tool. For that the command-oriented modelling technique many DEMapplications work with was transferred into an objectoriented approach. Especially for new users which are not familiar with discrete element method but also for old experienced users creating models and coupled simulations is getting very easy.

\section{References}

[1] Cundall P, Strack ODL. A discrete numerical model for granular assemblies.

[2] Otter M. Modelica-A Unified Object-Oriented Language for Physical Systems Modeling-Language Specification, 2000.

[3] Geimer M, Krüger T, Linsel P. Co-Simulation, gekoppelte Simulation oder Simulatorkopplung? Ein Versuch der Begriffsvereinheitlichung, O+P Ölhydraulik und Pneumatik, pp. 572-576, 2006

[4] Kunze G, Katterfeld A, Richter C, Otto H, Schubert C. Plattform- und softwareunabhängige Simulation der Erdstoff-Maschine Interaktion In: 5. Fachtagung Baumaschinentechnik, Dresden, 2012

[5] W. Noack. Der Entleerungsvorgang bei Becherwerken, In: Agrartechnik, 1955 


\title{
Design of Simulation Model for Novel Solar Thermal Storage Tank
}

\author{
Muthalagappan Narayanan*, Gerhard Mengedoht, Walter Commerell
}

Hochschule UIm, Prittwitzstraße 10, 89075 UIm, Germany; *narayanan@hs-ulm.de

SNE 27(3), 2017, 131 - 136, DOI: 10.11128/sne.27.tn.10382

Received: June 10, 2017 (Selected ASIM GMMS/STS 2017

Postconf. Publ.), Accepted: July 20, 2017

SNE - Simulation Notes Europe, ARGESIM Publisher Vienna,

ISSN Print 2305-9974, Online 2306-0271, www.sne-journal.org

Abstract. Due to the fluctuated availability of solar energy, the solar thermal energy systems are designed by simulation as to have a system which will satisfy the loads as expected. The thermal storage tanks are existing since a long time but however there is still research going on and new novel tanks are introduced. One such tank is the Hybrid Quattro thermal storage tank from Sailer $\mathrm{GmbH}$. This storage tank have new patented lances, which helps in stratification of the tank and thus higher efficiency of the energy storage without mixing of the layers in the tank. For designing of an energy system with this storage tank, a simulation model of this tank is required. Thus a test of this storage tank is done and the parameters were identified which later was used to build up a component model for storage in TRNSYS software using the type 340 storage tank model. This paper explains the process of identifying parameters from a real storage tank to a simulation model which can be used further for simulating a storage tank which would also make possible for the storage tank to be optimized for a development in the efficiency for the system performance.

\section{Introduction}

Currently Hochschule Ulm along with Sailer GmbH and other partners is working in the project Intelligent Home Energy Management (iHEM) funded by BMWi. The main objective of this project is to integrate thermal and electrical components with more energy sources at high efficient operation in a household for high self utilization.
For an intelligent control of such a system, the load and production energy are forecasted and an optimum predictive operation strategy is found out so that the system is not only efficiently optimized and self utilization prioritized with maximum possible renewable energy ratio but also economically and strategically viable. The subjective goal of the project is to have an integrated home energy system communicating with all the components through a superior management component.

Hochschule Ulm is involved in simulating different buildings with TRNSYS and finding out the design and sizing of different thermal and electrical systems like fuel cell CHP, gas boiler, solar thermal collectors, PV, thermal storage tank, battery such that the demand of the buildings are met. Also one final step of the project is to build a demonstrator with all these system components and present the intelligent control and integrated working of these. Also Hochschule Ulm is involved in design and monitoring of the demonstrator. More information about the project can be found at [1].

In project iHEM (Intelligent Home Energy Management), for system design the novel solar thermal storage tank from Sailer GmbH needs to be sized.

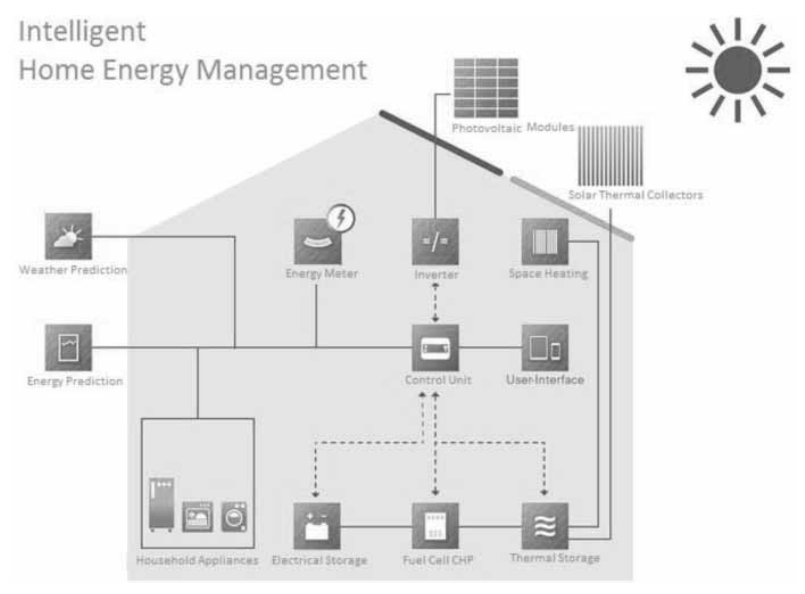

Figure 1. Overview of iHEM. [1] 
The storage tanks include patented lances which are efficient enough to form layers in the tank such that the temperature gradients inside the tank are maintained and hence avoiding mixing which results in efficient storage of heat. The first sub-motive of the project is to size the storage tank for design of the entire energy system.

Due to the fact that this is a complicated system, simulation of the system is required for designing the size of each component. For such simulations with high dynamic possibilities is the software TRNSYS (Transient System Simulation Tool) [2] famous. For the degree of freedom and its module plug and play concept, they are so flexible and customly designable. Each of the component in the system is a separate block in which the parameters can be defined. Also each of the output and input of the components can be interconnected so that they can communicate with each other. In TRNSYS there are lots of pre-developed models available which can be connected together and integrated as a system, just like in Matlab/Simulink. Furthermore, new component models can be coded and created. To design the system, the simulations have to be carried out with a storage tank model which would be able to simulate the Hybrid Quattro storage tank. Type 340 component model, pre-developed complicated tank component would be used here to model the tank. Below in section 1.1 and 1.2 the unique storage tank and the TRNSYS Type 340 model features are explained.

\subsection{Hybrid Quattro storage tank}

Usually a hot water storage tank is charged and discharged using fixed direct ports. So regardless of the temperature of the hot water produced by solar thermal collectors or the temperature in the layer where the port is, the hot water is injected in this layer and this in return mixes the tank layers and the temperature gradient inbetween the top and bottom is less. This causes the temperature of the discharge low. One more aspect is that the discharge return temperature depends on the application it is being used for and if return temperature is hotter and still useful this is also wasted by mixing the layers. For example when a solar collector produces $70^{\circ} \mathrm{C}$ hot water which is being fed into the top of the tank and after some time when the sun radiations are reduced the temperature reduces to say $50^{\circ} \mathrm{C}$ then this also is fed into the same layer in tank which mixes the layer and reduces the efficiency. For this the heat exchangers were built inside the tank.
The same applies for the discharging, when warm water is being used for space heating in buildings, the return temperature really depends on the demand of heat enery in buildings and the flow rate of the radiators. Also one more factor is that the discharging outlet temperature also depends on the temperature taken out from the tank for discharge to deliver energy.

Even though heat exchangers were better than direct charging and discharging, they were still not perfect and energy efficient as the heat exchangers cover only some layers and in these layers there may be a temperature gradient which might be destroyed. Thus came into existence, the stratification lances. These lances are mere plastic pipes which have openings at several layers inside the tank. The first kind of lances was developed by Solvis $\mathrm{GmbH}$. These lances are normal pipes which have openings at perpendicular angles in a right angle direction. The Solvis lances are shown in Figure 2 to the left. Also in the same figure you can see the Sailer lances in the right.

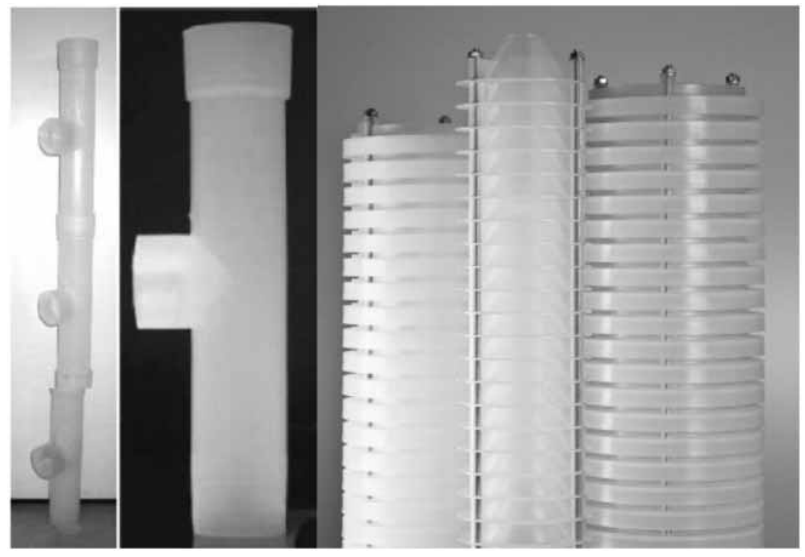

Figure 2. Stratification lances from Solvis $\mathrm{GmbH}$ (on the left) [3], and Sailer $\mathrm{GmbH}$ (on the right, where the middle lance is used for solar energy i.e. natural convection input). [4]

Definitely Solvis lances were a breakthrough but since it was right angled and directed to one point in a cylindrical layer, they did have some mixing. Hence were the newly developed Sailer lances, these had a 360 degree fool proof lances which had to take a convection fool proof design which made them even efficient. In a whole system point of view these tanks improve the efficient use of the energy system and higher useful energy ratio (useful energy/energy input). 
This combistore is claimed to be $60 \%$ more efficient than direct charging/discharging combistores by Sailer $\mathrm{GmbH}$ [5]. A comparison of the two stratification lances were made by Andersen et al. through CFD simulations, forced flow and the thermosyphon flow tests [6].

More efficient than the normal solar energy heat exchangers are the heat exchangers with stratification lances concept used here by the hybrid quattro tank. Since the heat exchangers if installed alone, the stratification is disturbed and when only lances are used glycol solution cannot be used in the tank.

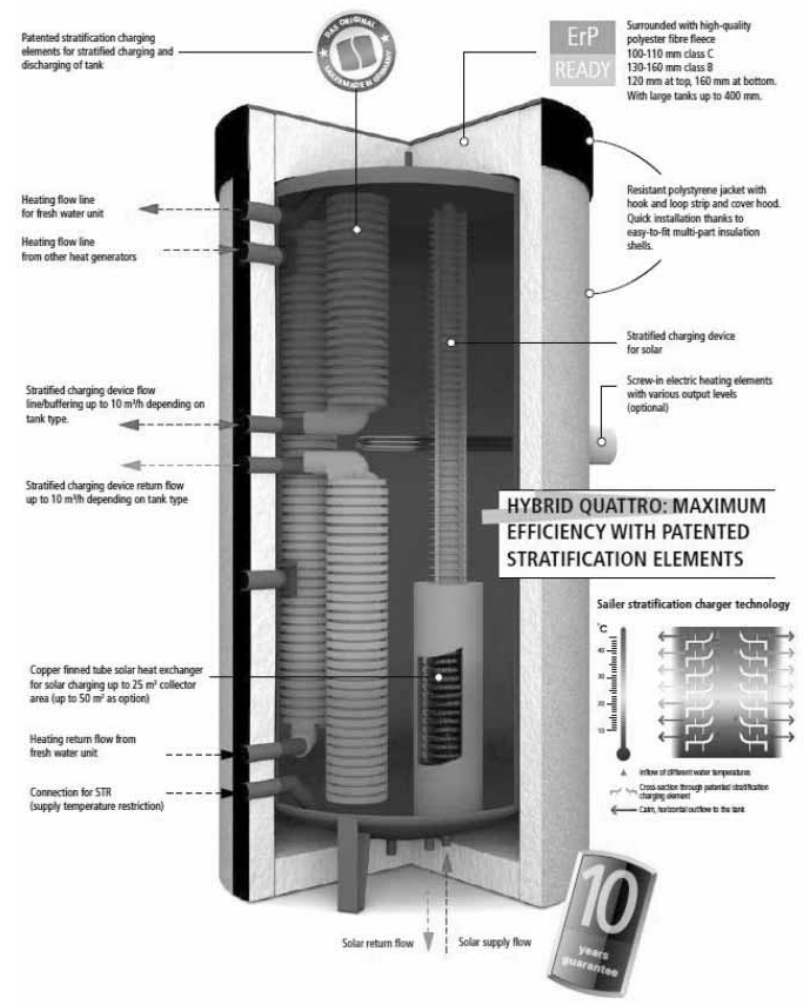

Figure 3. Sectional view of the Hybrid Quattro tank with the stratification lances. [5]

Until now tanks used an external heat exchanger which transfers heat from glycol loop to the water loop and the hot water is fed into the tank with lances. But here they use the natural convection of the water and the density in the heat exchange-lance combo. The heat exchanger inside the tank brings heat and transfers it to the nearby water and due the fact that this water in the enclosure is high of density it moves via the lance to the respective layer and the cold water flows in the enclosure of the heat exchanger from the bottom to absorb the heat from the glycol-water solution from the solar collectors.

\subsection{TRNSYS Type 340}

The TRNSYS Type 340 is a storage model for a multiport tank component. This was developed by ITW, UniStuttgart and is a non standard model available for purchase and can be easily integrated into the TRNSYS Library. The storage tank has a provision for up to 10 lances and 4 heat exchangers with up to 200 nodes (same temperature layers) and an electrical auxiliary heater. In Figure 4, it can be seen that the model is very flexible and is mathematically coded.

The number of nodes can be input and they are equally divided from the input height and volume. The tank heat loss can be either given as a value same in all directions or can be varied between different parts of the store. The heat exchanger and the lances can be combined to have a heat exchanger which will send the hot fluid via lances to the stratified layer. Also all the lance's input height, output height, flow direction and also if it there is a lance (stratified or direct ports) can be given. Also the heat transfer capacity rate has to be put in. There is also the facility to have one internal electrical auxiliary heater.

There are also some limitations to the model. It cannot have a lance with only one inlet/outlet port. It is assumed in the model that the mass flow in one lance, i.e. in the outlet and inlet is meant to be a constant value.

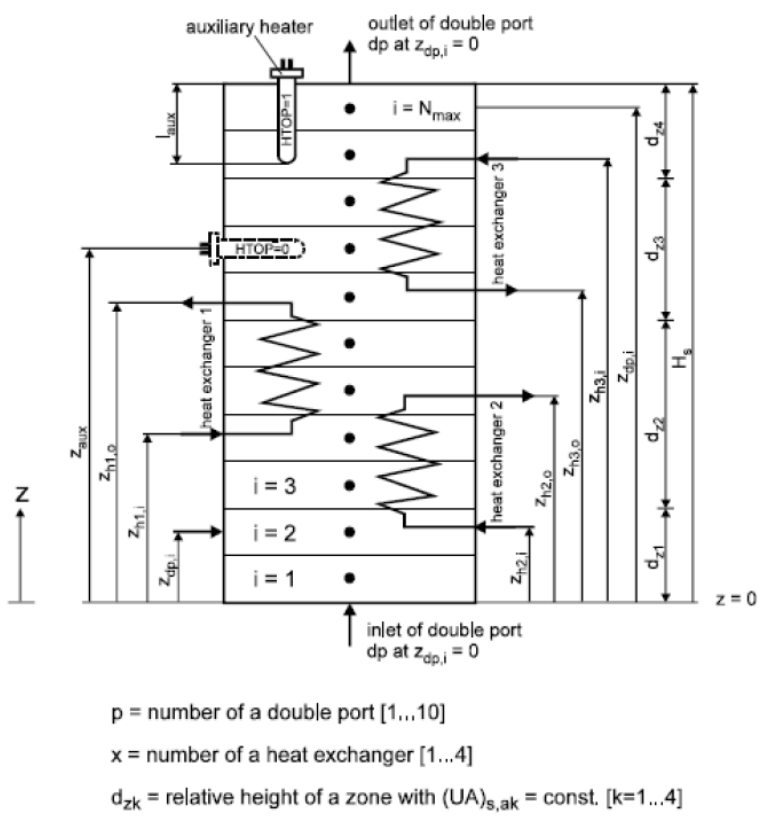

Figure 4. Schematic of the multiport store model as it is in TRNSYS type 340 component model. [7] 
So always the lances should be connected to a closed loop in which the mass flow of the inlet and outlet of the lances should be same. Each of the heat exchangers is substituted for a lance and if one uses the maximum available 4 heat exchangers then there are only 6 lances available for the connection. This is because whenever a heat exchanger is connected, there arises sometimes a need to have a lance connected to this heat exchanger. Thus mathematically either of the heat exchanger or the connected lance could only be used.

\section{Methodology}

As there are different types of hot water storage tanks and there are diferrences in how they perform a common test method in which parameters can be identified in order to compare them is required. Also for simulation of these tanks and designing a whole energy system these parameters are required. As a development of IEA Task 16, a European Standard EN 12977-4 was formed for performance test analysis of solar combistores.

According to EN 12977-4, as specified in [8], there are different types of solar thermal storage tanks and briefly combistores can be classified into 5 types with direct/indirect charge and discharge modes. In this case for Hybrid Quattro tank, the charge ports are both direct and indirect while the discharge ports are only direct discharge. There are fresh water station heat exchangers externally which also have to be taken into account. Depending on the type of the combistore a different test methodology is specified and the tests are done in two types-entire store and a certain volume (auxiliary part) to determine heat loss capacity rate and thermal stratification loss (effective vertical thermal conductivity) respectively. Other than this the whole procedure gives many additional useful parameters like effective volume, heat transfer capacity rate, stratified charging capacity, etc. And the end of post processing of the test results with parameter fitting, the parameters for the respective tank are identified. The other physical parameters like inlet and outlet port heights, lances length, tank volume, height and sensors, heat exchanger and lances performances etc. can be obtained from the manufacturer. This standard testing can be done in any authorized test center that has this combistore test bed.

For this case, a $2 \mathrm{~m}^{3}$ Hybrid Quattro Sailer GmbH's Combistore Tank was tested by ITW, Uni-Stuttgart and the performance parameters were identified. The challenge then is to design a model to simulate such a complex storage tank.
Not only in this project is it looked to simulate the storage tank but also to find optimal tank parameters for different energy systems and buildings. The optimization of tank includes varying the height of the inlet and outlet ports, volume of the tank, electrical heater placement, electrical heater power, insulation, etc. Hence also the store size has to be varied and hence the parameters such as heat loss rate would vary and influence the simulation results. As known, the heat loss rate varies with respect to the insulation thickness, insulation material and surface area of the storage tank.

Therefore basically the insulation can assumed to be varied in such a way that the surface area alone varies the heat loss rate, so that the magnification of the combistore for simulations is viable. The effective vertical thermal conductivity increases as the tank size decreases but as they are small in context they are neglected. Overall when there is a need to increase the size of the combistore, the height and the volume is modified with the heat loss modified and all the other paramters are withheld the same.

\section{Simulation Realization}

Now with the test results done and the parameters identified, the task following is to bring in these to the simulation model. As already explained TRNSYS software is used and type 340, a multiport storage model is used. Even though this is one of the best component model for storage tanks, it has some limitations. In Figure 3, it can be seen that there are two ports in the middle of the tank which is connected to the stratification lances. The main idea of these lances are that these two lances can be used in for two different operations together as shown in Figure 5.

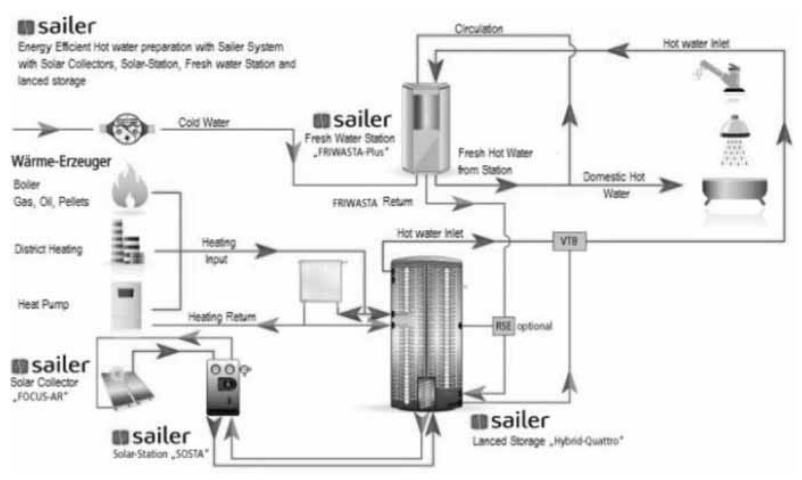

Figure 5. Schematic of the Sailer hybrid quattro tank as it is functioning in the real system. [9] 
In most of combistores there is an auxiliary boiler and it is also connected to radiators for space heating. The principle of the radiators operation is that it takes out warm water from the middle of the tank i.e. from the top of a lance and returns back to the bottom of the same lance which inserts the water in the relevant temperature. Vice versa, for the auxiliary boiler, in this project a gas boiler, the warm water is taken from the middle of the tank i.e. from the bottom of another lance and is heated. The hot water from the gas boiler is fed back to the tank via the top of the same lance so that the hot water reaches the layer with relevant temperature.

One more advantage of this configuration is that during the winter days, without using the tank for buffer, the gas boiler can directly power the space heating radiators. One necessary equipment for this setup is a two way pump which is missing in TRNSYS library. Most importantly, type 340 is modelled or coded in such a way that always the input and output of each lance is two different ports and only with the direction of the lance, the component model defines if it is charging in energy or taking out. Also it is designed that the mass flow in between these input and output port is constant and hence if one of the port is not connected, it gives an error. Therefore it is practically possible to use a stratification lance with just one port but mathematically, not just in TRNSYS for type 340 but also in any other platform, this is not viable. Therefore this configuration setup has to be varied in simulation which differs from reality but the principle of the lances is still feasible. The difference in the simulation model to reality can be seen in Figure 6. One more similar change is that the lances are connecting the fresh water station.

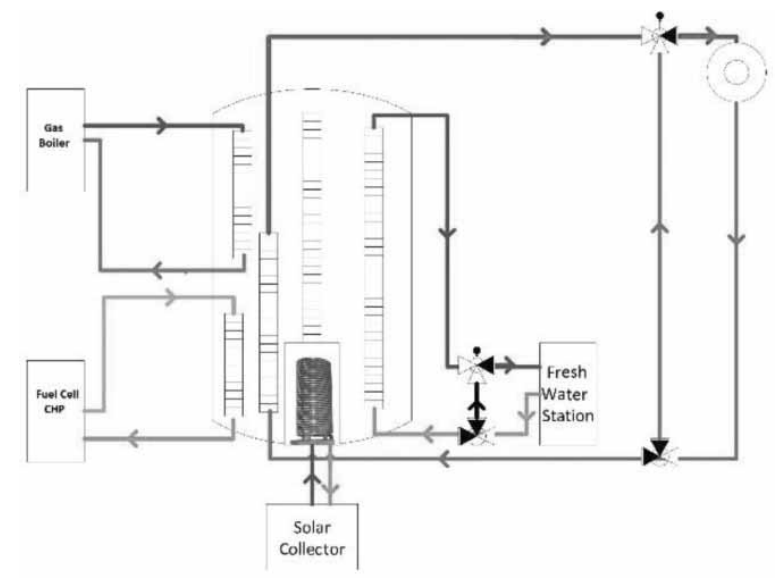

Figure 6. Overview of the simulation setup for the tank model.
The real operation is that the fresh water station takes hot water from the top of the tank via a direct port and exchanges the heat to the fresh cold water and the return warm water is fed to the lances which in turn delivers to the respective layer in the tank. Instead of this setup in the tank the inlet and outlet to the fresh water station is connected to the single lance.

\section{Results and Conclusion}

The primary aim of the paper is to build a component model for the Hybrid Quattro Storage Tank from Sailer $\mathrm{GmbH}$. The process for building a simulation model for a storage tank is presented in this paper. Since the storage tank is a novel tank with the stratification lances, the parameter identification and simulation turns out to be challenging. With certain conceptual changes between the real system and the simulation model, the simulation model is executed.

The advantage of the simulation model is that the size of the tank can be varied and can be looked into for various cases. The simulation model also is helpful to vary the port inlet and outlet heights according to the system so that the total system efficiency is high. In a following task as part of the iHEM project, the developed simulation model is used to build an energy system model consisting of solar thermal collectors, gas boiler, fuel cell CHP, building heat load and domestic hot water load. Also PV, battery and inverter are modelled so that the electrical auxiliary heater in the hot water storage tank is supplied with the excess electrical energy.

Finally the motive of the project is to compare the different buildings in Germany and the strategy for such a home energy system and the sizing. For example, for a sun house standard, the solar fraction needs to be minimum 50\% while for a KfW55 house the primary energy used in the house should be less than $55 \mathrm{kWh} / \mathrm{m}^{2}$. Thus the collector area, PV size, storage size, etc. are different for different buildings and the approach for each building types's system optimization is different. Not only that but also the tank also needs to be optimized for each of the building type according to their energy requirements and system size. 
The fact that Sailer GmbH has the option to build custom-made different tanks according to the requirements of each customer is an advantage. So the end result might be to find a tank design and a sizing factor for each of the building types. Also there is a real running system of all these components, and their management strategies and control would be realized in a demonstrator system at the end of the project.

\section{Acknowledgement}

The work described in this paper is a part of the project Intelligentes Heimenergiemanagement (iHEM) and the Research Initiative Energiespeicher funded by the Bundesministerium für Wirtschaft und Energie under the Project Number -03ET1205A.

\section{References}

[1] Photovoltaik Augsburg: Solarthermie, Photovoltaikanlage - iHEM. [Online]. Available: http://www.ihem.eu/. [Accessed: 13-Jan-2017].

[2] Transient System Simulation Tool - TRNSYS. Madison USA.: Thermal Energy System Specialists, LLC., 2014.

[3] Shah LJ, Andersen E, Furbo S. Theoretical and experimental investigations of inlet stratifiers for solar storage tanks. Appl. Therm. Eng., vol. 25, no. 14-15, pp. 2086 2099, Oct. 2005.
[4] Sailer GmbH Ehingen - Patented stratification charger technology. [Online]. Available: http://www.sailergmbh.de/en/products/sailerstratifiedstorage-tanks/patented-stratificationcharger- technology.html. [Accessed: 13-Jan- 2017].

[5] Sailer GmbH Ehingen - Schichtenspeicher. [Online]. Available: http://www.sailergmbh.de/en/products/schichtenspeicher / schichtenspeicher.html. [Accessed: 13-Jan-2017].

[6] Andersen E, Furbo S, Hampel M, Heidemann W, Müller-Steinhagen $\mathrm{H}$. Investigations on stratification devices for hot water heat stores. Int. J. Energy Res., vol. 32, no. 3, pp. 255-263, Mar. 2008.

[7] Drück H. Mutliport Store Model for TRNSYS -Type 340. Institut für Thermodynamik und Wärmetecknik (ITW), Universität Stuttgart, Mar-2006.

[8] FprEN 12977-3:2016, Thermal solar systems and components - Custom built systems - Part 4: Performance test methods for solar combistores.

[9] Sailer GmbH Ehingen - Diagram: Hybrid Quattro in heating circuit. [Online]. Available: http://www.sailergmbh.de/en/products/sailerstratifiedstorage-tanks/diagram-hybrid-quattroin-heatingcircuit.html. [Accessed: 13-Jan-2017]. 


\title{
Conceptional Problems of Transaction-based Modeling and its Implementation in SimEvents 4.4
}

\author{
Lars Austermann *, Peter Junglas, Jan Schmidt, Christian Tiekmann
}

PHWT Vechta, Schlesier Str. 13, 49356 Diepholz, Germany; * peter@peter-junglas.de

SNE 27(3), 2017, 137 - 142, DOI: 10.11128/sne.27.tn.10383

Received: June 10, 2017 (Selected ASIM GMMS/STS 2017

Postconf. Publ.), Accepted: July 20, 2017

SNE - Simulation Notes Europe, ARGESIM Publisher Vienna,

ISSN Print 2305-9974, Online 2306-0271, www.sne-journal.org

Abstract. Transaction-based modeling is a widely used graphical method for modeling discrete event systems, a recent implementation being SimEvents from Mathworks. Though it is applicable to a wide range of problems, it has its specific drawbacks. Some of them are connected to the basic abstractions of the method, others are related to the specific program used. By implementing a few different standard examples, we will show some of these shortcomings together with possible workarounds. This should be pointed out, when teaching this method to new users, but additionally has to be taken into account, when building a new transactionbased library or corresponding blockset.

\section{Introduction}

The modeling of discrete systems is a broad and difficult subject that has created a wealth of very different paradigms. Concentrating on graphical methods only, widely used techniques range from highly abstract ones such as Petri nets [1] and state graphs [2] to concrete material flow applications, e. g. PlantSimulation [3].

At a medium level of abstraction one finds processbased and transaction-based modeling [4], which both describe entities that are handled by fixed components. A widely used implementation of the former method is Arena [5], of the latter SimEvents [6]. The basic difference between them is the way how entities are transported: In process-based methods the components are the active parts and 'seize' the entities, in transactionbased systems the entities play an active role and move automatically, until they are blocked.
These two methods are used in many industrial applications, since they are sufficiently abstract to be universally applicable, but at the same time concrete enough to be comprehensible by users from very different disciplines.

But until now there is no well-established set of basic features and components for both methods. As a consequence users have to stick to a given simulation environment or even to a fixed version, if they don't want to reimplement and partially redesign their models. As the huge conceptional changes between the latest releases of SimEvents indicate [7], especially the definition of the transaction-based modeling approach presently seems to be unclear. Therefore one should reconsider the basic design of the method and try to answer questions such as the following: What are the shortcomings of current implementations? Which concepts or components are missing? How could a reasonable set of components be defined?

The aim of this paper is to provide first steps to answer these questions. To this end it examines a few standard example problems and their implementations in SimEvents 4.4. It shows the conceptual problems that have been encountered during the implementation and provides possible workarounds. It concludes with some proposals related to applying and teaching the transaction-based approach to modeling.

\section{Example Models}

To study the range of applicability of the transactionbased approach, one can draw on a huge stock of practical examples. For the purpose of finding the weaknesses of the method, four models will be presented in the following, that clearly indicate the points that are of interest here. The first three come from Law's textbook [8], the last one from the ARGESIM benchmark C14 [9]: 
- timeshared: Model of a time-shared computer, where several terminals send jobs of varying computing time demands, which are processed in time slices using a round-robin scheduler.

- multiteller: Model of a multiteller bank with several queues and jockeying, i.e. customers are allowed to change to a shorter queue.

- jobshop: Model of a factory with five workstations, where variable kinds of jobs are processed, which require different paths through the stations.

- supplychain: Model of a supply chain consisting of wholesalers, who order different products from distributors, which in turn order from several fac-tories. The distributors use special strategies to comply with the demand.

The models are described in full detail in their references. The following section will concentrate on those parts of the implementations that are relevant for the discussion.

\section{Problems Implementing the Examples}

In the following some of the conceptual problems that have been encountered during implementation of the examples will be presented in detail. In addition it will be shown how to cope with them, mainly by introducing components that help to implement new abstractions.

\subsection{Handling of concurrent events}

The central element of many discrete event systems is a global event queue that contains all events in the proper order. But in a transaction-based model the events are defined locally by the individual blocks, so that the proper order of events that originate from different blocks at the same time instant is not always welldefined.

An example is shown in Figure 1: A generator component creates entities with increasing ids, which will be routed afterwards to one of two outputs depending on whether the id is even or odd. For this purpose the Get Attribute block extracts the id of an entity, the Compute Port component uses this value to compute the corresponding output port number and the SetAttribute block sets the port number as an attribute of the entity.

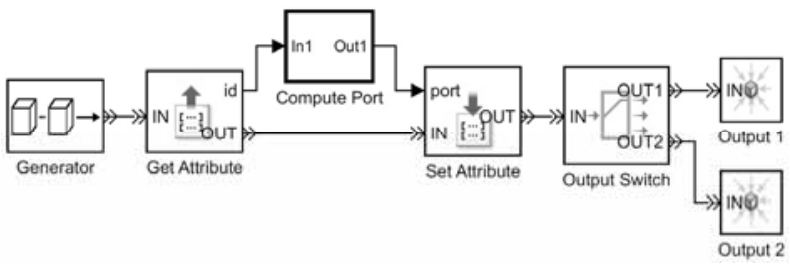

Figure 1: Example with concurrent events.

Simulating the model leads to an error message stating that a race condition has been detected; it is not defined which of the two inputs of setAttribute arrives first: the new entity or the new attribute value. If one ignores this message by changing it into a warning, the entities leave the Output Switch at the wrong port. But if one follows the recommendation given in the error message and inserts a null server, i.e. a Server component with service time $t_{s}=0$, after the entity output of Get Attribute, the model works as planned.

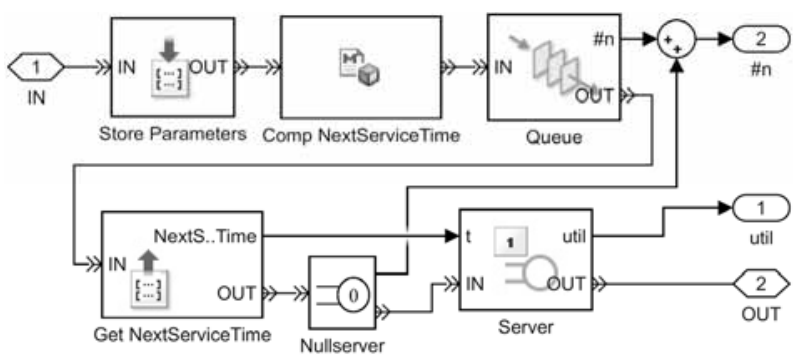

Figure 2: CPU component of the 'timeshared' example.

This problem is inevitable when working with local components and is of course well known for a long time: already the textual modeling language GPSS had a BUFFER command that reorders concurrent events [10].

The insertion of a null server to delay entities seems to be a simple solution, but it has a serious drawback: If its output is blocked, the server stores one entity. This has to be taken into account properly and complicates model design, as can be seen in the implementation of the CPU in the 'timeshared' example (Figure 2).

When jobs leave the queue to enter the server block that represents the CPU proper, their service time has to be fetched from an attribute, which makes the in sertion of a null server necessary. If one is interested in statistical data about the length of the queue, one has to add the additional job that may be stored in the null server. Therefore the average queue length that the queue component provides, is of no use, it has to be computed 'manually' instead. 
The workaround of using a null server to implement an 'infinitesimal' delay is conceptually wrong, because the implicit storage that it adds, is not related to the problem it solves. This can even lead to more serious problems as will be seen in Section 2.4.

\subsection{Separation of entities from a queue}

The 'multiteller' example allows for the well known phenomenon of jockeying in a queue, i.e. customers at the end of a queue can switch to another shorter one. Modeling this behaviour with SimEvents turned out to be much more difficult than expected. The reason is that although an entity can leave a queue, when its waiting time exceeds a threshold (a behaviour known as reneging), there is no way to detach the last entity of a FIFO queue on arrival of an input signal (e. g. when another queue has become shorter).

To model such a jockey queue two very different schemes have been devised: In the shuffle queue (Figure 3) the signal opens up a path from the exit to the beginning of the queue. All entities walk around the circle and get back into their old position, except for the last one, which leaves the block through the extra jockeying output. From here it is routed to the shorter queue.

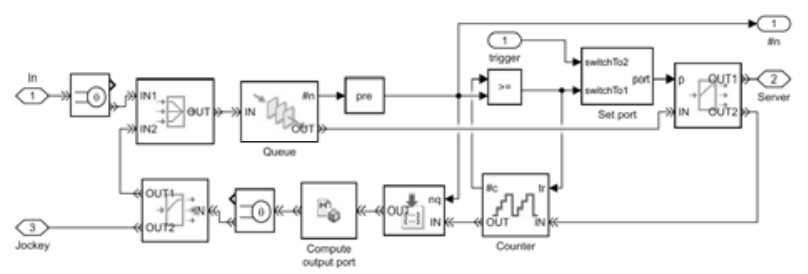

Figure 3: Shuffle queue component of the 'multiteller' example.

The clone queue (Figure 4) creates duplicates of all incoming entities and routes them into a FIFO and a LIFO queue. When the queue exit is opened, an entity is taken from the FIFO queue, but when the jockey signal arrives, the LIFO queue is used. A bookkeeping device destroys clones, whose partner has already left the queue, when they appear at the end.

Both schemes are quite complicated and lead to a lot of additional events. Though they worked at last, their implementations had to cope with a lot of difficult timing problems and appear to be cumbersome and error prone. What one needs instead, is a simple mechanism to remove a given entity from a queue, maybe similar to the concept of user chains in GPSS [10].

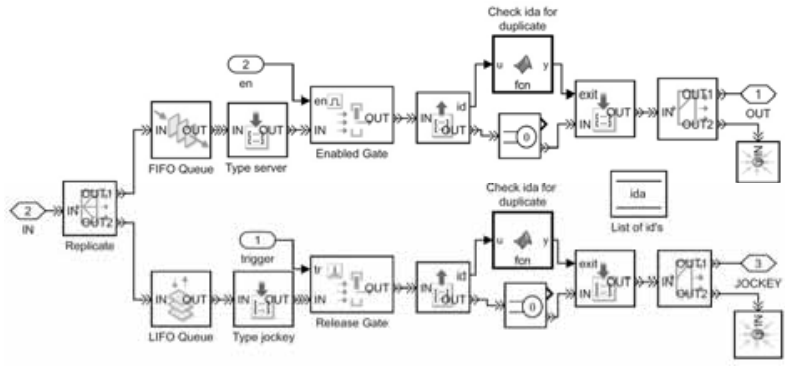

Figure 4: Clone queue component of the 'multiteller' example.

\subsection{Storing entities}

A basic ingredient of the 'supplychain' example is a storage component that stores incoming entities denoting products of several types. On arrival of an order it emits the corresponding products at its output port. Scanning through the SimEvents library to find blocks that can store elements, one comes up with the queue, the server and the resource blocks, but none of them seems appropriate for the task at hand: a server is not well suited to deliver a certain type of product on demand, the resource pool provides a fixed amount of resources. And the queue block doesn't scale well with the number of different product types, since one needs one queue per type to emit a product entity of given type on request.

Instead of trying to use some of these blocks together with complicated gates and logic to coerce them into a non-fitting scheme, one can use a simple $1 / z$ block from Simulink's basic discrete library. It contains the inventory (Figure 5), which is just a vector with the amounts of the different product types in the stock.

The Storage component (Fig. 6) registers incoming products in the inventory and destroys the corresponding entities. When an order arrives the inventory is reduced and the proper entities are recreated at the output port.

Of course this is only a trick, because the entities themselves are not stored at all. It worked for the example program, since the structure of the product entities is simple and always the same. What one really needs, is a more general component that actually stores the incoming entities and can release them on demand. The actual design of such a block is open to discussion and could be guided on example models and implementations in other environments. 
A simple first idea would be to use a special queue, where selected entities can move to the front of the line. Again, the old idea of 'user chains' could come in handy here.

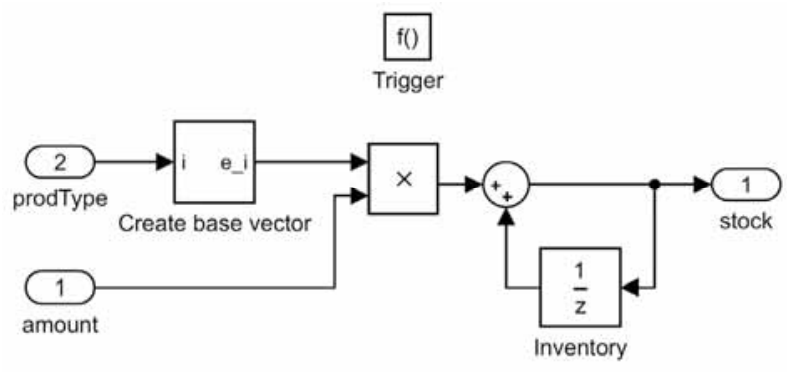

Figure 5: Change Stock component of the 'supply chain' example.

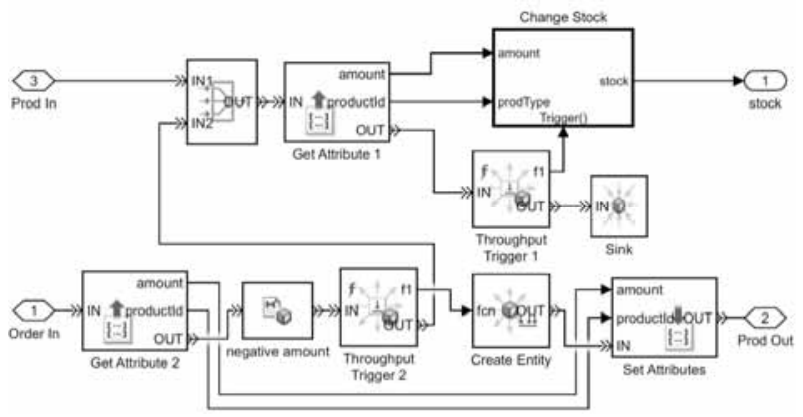

Figure 6: Storage component of the 'supply chain' example.

\subsection{Time measurements across several blocks}

In the 'jobshop' example entities use different paths through workstations with associated queues. One is interested in statistics for the total waiting time of the entities over all those queues. To measure this value, one needs timers that can be paused after a queue and resumed before the next one to add up the single waiting times of passing entities.

Unfortunately SimEvents only provides simple timers that measure between two fixed points. Therefore one has to add up the waiting times of the individual queues inside the entity using an attribute (Figure 7).

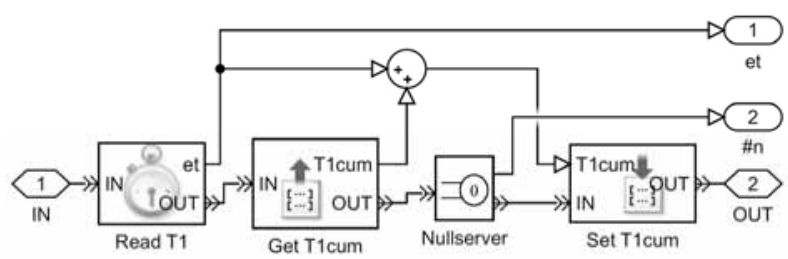

Figure 7: Accumulation of times in a PauseCTimer component.
Based on this idea one can easily build components to start, pause, continue and read such accumulating timers and test them in simple models (Figure 8).

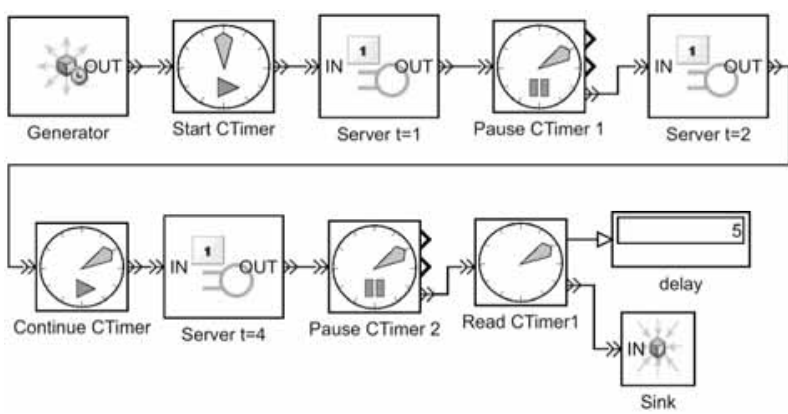

Figure 8: Simple test model for accumulating timers.

But the problem is, they don't work if the accumulating block PauseCTimer sits between a queue and a server which is exactly the most interesting configuration in general and especially in the 'jobshop' example. The reason is of course the null server block that is necessary inside PauseCTimer: When the server is busy, the first entity in the preceding queue moves into the null server and waits there instead in the queue. The additional waiting time is not accounted for in the timer. This is a serious problem: One could measure the time that an entity stays in the null server, but to accumulate it, one needs another null server!

As always there is a workaround: Accumulation between a server and an (unlimited) queue is no problem, as the queue never blocks and the preceding null server doesn't store an entity. Therefore one can measure the time $t_{Q+S}$ between entering the queue and leaving the server and the time $t_{S}$ inside the server and accumulate the time $t_{Q}=t_{Q+S}-t_{S}$ after the server. But this is akward and only shows again that the basic design of a null server is seriously flawed.

\subsection{Statistical analysis}

An important aspect of discrete simulation is the gathering of statistical data, often in the form of a final report. In the 'jobshop' example for instance, one is interested in the queue delay, queue length and server utilisation for the different workstations, as well as the total waiting time per job and per jobtype.

In a transaction-based environment there is no instance to collect such data but the blocks themselves. SimEvents provides mean values, utilisations and similar data for individual components, but no additional statistical tools. 
This is unfortunate, because due to the abundance of null servers one often has to combine individual values and can not use the statistical block data itself. The actual length of a queue for example has to be enlarged by the occasional inhabitant of a subsequent null server, as well as the corresponding waiting time. To compute mean values per entity or per time one has therefore to build own blocks, which admittedly can be done easily with standard Simulink methods.

Another challenge is the collection of statistics connected to entities, not blocks, such as the accumulated waiting times through several queues. The basic idea here is to store data in the entities themselves and collect them at the end. Figure 9 shows how mean or maximal values can be computed with the help of simple $1 / z$ blocks that are hiding in the Adder and Max blocks.

Combining all these auxiliary blocks in a final statistics subsystem not only helps to unclutter the model, but brings together all statistical data. They now can easily be pipelined into a Matlab script that creates a proper report file, if such is desired.

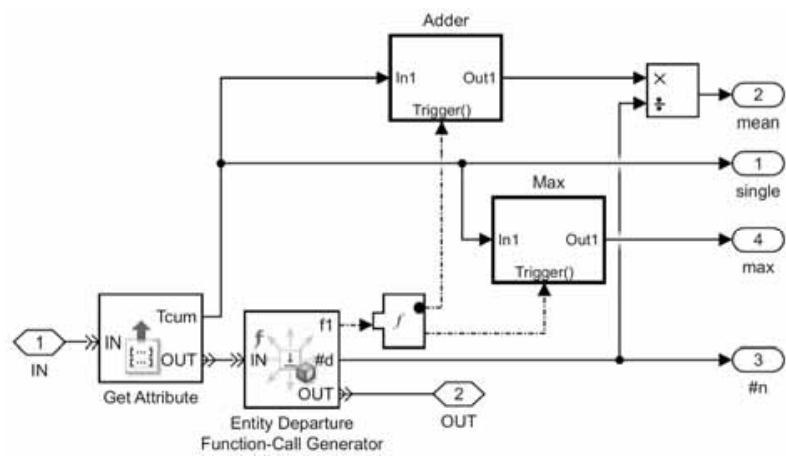

Figure 9: Component Ent ityMeanMax for accumulating entity data.

\section{Conclusions}

The preceding analysis has unfold four different areas, where transaction-based modeling and its implementation in SimEvents show conceptional difficulties:

- timing of concurrent events;

- implementation of alternative queueing policies;

- storing and retrieving of entities;

- gathering and processing of statistical data.
Of these the first one is by far the most serious, and a generally working solution instead of the defective null server workaround is not available in SimEvents. This is especially annoying since already GPSS had a better solution with its BUFFER command.

All other problems could be solved by introducing appropriate subsystems using blocks available in SimEvents or the underlying Simulink. Adding such components to a user library, one can simplify the modeling of a wide range of applications. Though this may be sufficient for the practitioner in the industry, it is a real drawback of the transaction-based method: a corresponding library should provide the basic abstractions that are necessary to model all problems that the method adresses.

Considering the queueing and storing problems the GPSS construction of 'user chains' seems to be a promising idea: Instead of adding ever more specialized components, it provides an underlying mechanism that may be able to cope with some of the difficulties presented above. To gain further insight into possible solutions, we propose to add a new benchmark to the ARGESIM suite [11] that requests the modeling of several jockeying queues and the collection of statistical data including the delay over several queues, similar to the multiteller example. To complicate matters one could ask for implementations with a large number of queues, which would adress the problem of 'vectorising' components.

Mathworks has realized that SimEvents 4.4 has still basic problems and came up with a complete redesign of its SimEvents library with version 5 . A very interesting feature is that the design is based on a unifying theoretical description [7]. Unfortunately, Mathwork has chosen a new design instead of relying on the wellknown DEVS formalism [4]. Many basic aspects have been changed with the new release, often by substituting graphical elements with Matlab code. As a consequence there is no simple migration path from the old to the new version. Whether this has lead to a satisfactory implementation, needs further investigation.

In any case this only adds to the central point made here: For the advancement of transaction-based modeling it is vital that it is based on a thorough theoretical analysis to reveal the fundamental abstractions and basic components that are necessary. 
The goal is to find stable designs, that don't change with every new tool or release, to get models that are better understandable, because they don't rely on tricky workarounds, and to reach high quality solutions, since they have a sound foundation. If we don't care for the basic concepts, we have to live with redesigning our models and rewriting our lectures every other year.

\section{Acknowledgements}

The second author (P. J.) likes to thank Thorsten Pawletta for introducing him to the rich history of discrete modeling tools.

\section{References}

[1] Cassandras CG, Lafortune S. Introduction to Discrete Event Systems. Springer, New York, 2. ed. 2008.

[2] Harel D. Statecharts: A Visual Formalism for Complex Systems. Science of Computer Programming, 8, pp. 231274, 1987.

[3] Bangsow S. Tecnomatix Plant Simulation: Modeling and Programming by Means of Examples. Springer, Cham, 2015.
[4] Zeigler BP, Praehofer H, Kim TG. Theory of Modeling and Simulation. Academic Press, San Diego, 2nd ed. 2000.

[5] Kelton WD, Sadowski RP, Zupick NB. Simulation with Arena. McGraw-Hill, New York, 6. ed. 2015.

[6] The MathWorks. SimEvents: Model and simulate discrete-event systems. Online: www.mathworks.com/products/simevents.html (called 2017-01-30).

[7] Li W, Mani R, Mosterman PJ. Extensible discrete-event simulation framework in SimEvents. Proc. 2016 Winter Simulation Con-ference, Arlington, pp. 943-954, 2016.

[8] Law AM. Simulation Modeling and Analysis. McGrawHill, New York, 5. ed. 2014.

[9] Tauböck SM. C14 Supply Chain Management Definition. Simulation News Europe, 32/33, pp. 42-43, 2001.

[10] Schriber TJ, On the application of user chains in GPSS. Proc. 1973 Winter Simulation Conference, San Francisco, pp. 140-158, 1973.

[11] Breitenecker F, Wassertheurer S, Popper N, Zauner G. Benchmarking of Simulation Systems-the ARGESIM Comparisons. Proc. First Asia Int. Conference on Modelling \& Simulation. pp. 568-573, 2007. 


\title{
Automatic Layout of Scilab/Xcos Diagrams
}

\author{
Chenfeng Zhu ${ }^{1 *}$, Umut Durak ${ }^{2}$, Sven Hartmann ${ }^{1}$, Clément David ${ }^{3}$ \\ ${ }^{1}$ Clausthal University of Technology, Department of Informatics, Julius-Albert-Straße 4, \\ 38678 Clausthal-Zellerfeld, Germany; * chenfeng.zhu@tu-clausthal.de \\ ${ }^{2}$ German Aerospace Center (DLR), Institute of Flight Systems \\ ${ }^{3}$ Scilab Enterprises
}

SNE 27(3), 2017, 143 - 151, DOI: 10.11128/sne.27.sw.10384 Received: June 8, 2017 (Selected ASIM GMMS/STS 2017 Postconf. Publ.), Accepted: July 20, 2017

SNE - Simulation Notes Europe, ARGESIM Publisher Vienna, ISSN Print 2305-9974, Online 2306-0271, www.sne-journal.org

Abstract. Scilab/Xcos is a graphical modeling and simulation environment for hybrid dynamic systems. It provides a graphical editor which allows representing models with block diagrams. While each block represents a computational function, links specify the data and event flow. However, as the number of the blocks and the links increases, the Xcos schema can quickly become messy and difficult to read. In this paper, we present an approach for automatically updating the layout of an Xcos schema by manipulating the links and the split blocks, so that the diagrams can be kept well-presented and readable. In this approach, we update the link styles with an optimal route and then, rearrange the positions of blocks. The proposed approach is exemplified with sample Xcos models. In addition to providing the automatic layout capability to the Scilab/Xcos user, an application programming interface is also specified for the Scil$\mathrm{ab} / \mathrm{Xcos}$ developer who want to further enhance the provided feature set.

\section{Introduction}

Scilab is a free and open source software about numerical computation for engineering and scientific applications [1]. Xcos is the graphical modeling environment of Scilab for modeling and simulation of hybrid dynamical systems [2].

When using Xcos to create models, we often create blocks to implement computational functions and use links to connect them for data and event flow. They are all well-organized at the beginning as we start building up the model.
However, as the model becomes more and more complex, and the number of blocks increases, we require layout rearrangements more frequently; we start moving the blocks or links every now and then. But manual layouting is hard, labor intensive and error prone. Such an effort usually ends up with a model in a messed up layout, which makes the diagram ugly and difficult for modification. Thus the readability and the maintainability of the model is decreased. Figure 1 is an example of a disordered Xcos diagram.

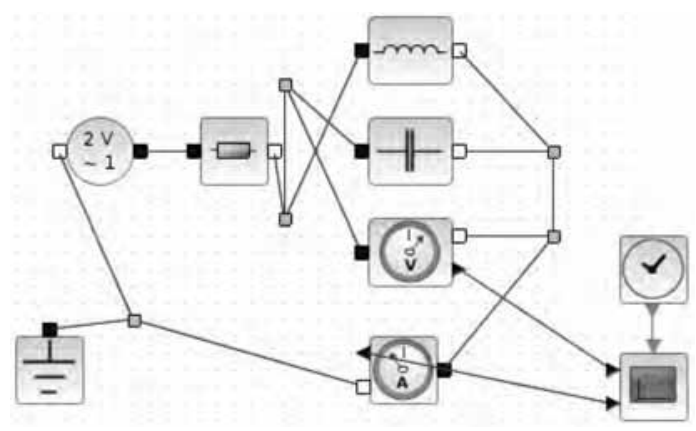

Figure 1: A Sample Xcos Model.

A constant manual work for relocating the blocks and rearranging the links between blocks is required in order to maintain the model readability. While block positioning is relatively straight forward and even may be more efficient manually, the link rearrangements are hard and cumbersome. This effort is about developing the capability for automatically improving the model layout by manipulating the links and split blocks that connect the links to each other, thereby keeping the model readable. The Optimal Link Style (OLS) that is introduced in the Section 3 proposes an optimal route for a link which could make the link clear in the diagram. In the Section 4, the Split Block Automatic Position (SBAP) that rearranges split blocks in better positions is presented. Lastly in Section 5, the conclusion is presented and we discuss future work for a better automatic Xcos layouting. 


\section{Related Work}

\subsection{Graph Theory}

For finding the optimal link between two blocks, one can apply a graph search based approach. The approaches from the graph theory to find the shortest route with the minimum cost are already quite mature. The Dijkstra's algorithm is an algorithm for finding the shortest paths between nodes in a graph, which was conceived by computer scientist Edsger W. Dijkstra in 1956 and published three years later [3, 4]. The Bellman-Ford algorithm is an algorithm that computes shortest paths from a single source vertex to all of the other vertices in a weighted digraph [5]. The FloydWarshall algorithm is an algorithm for finding shortest paths in a weighted graph with positive or negative edge weights (but with no negative cycles) [6, 7]. These theories are practical and useful for solving the shortest path problem which could also be extended to solve the minimum-cost problem. However, in modeling graphical model, there are always more than one alternative for the connection which look good. And sometimes, some links which look good and readable are not the shortest ones.

As to the problem about the positions of split blocks, we could use some basic graph drawing theories to reorder blocks. For instance, pseudo hierarchical tree and vertical or horizontal aligned layout would be helpful to make diagrams easy to read and clear to maintain. But for both cases, our approach to the problem was to develop heuristics that capture user insight for readability.

\subsection{Layouting in MATLAB/Simulink}

Other graphical modeling environments such as MAT$\mathrm{LAB} /$ Simulink also provide capabilities for formatting the layout of their own diagrams. Simulink is also a graphical editor for Model-Based Design which provides customizable block libraries, and solvers for modeling and simulating dynamic systems [8]. Compared to the automatic layouting of other graphical modeling environments, Simulink achieves quite an outstanding work about this. It not only provides the beautiful layout, but also could implement the dynamic features. Simulink can automatically find the 'optimal path' so that the new signal line is as short as possible, has minimal 90 degree turns, and does not overlap other blocks and text.
Moreover, as you draw the signal line, Simulink lets you know exactly what the path is going to look like before you release the mouse button [9]. Additionally, Simulink provides one-click to beautify the model diagrams and autoarrangement the blocks and lines when building new functionalities [10].

\subsection{Layouting in Scilab/Xcos}

In fact, even in Scilab/Xcos, it is possible to improve the general look of a diagram in using the blocks alignment options and the links style [11]. Besides straight style and the free style with control points, there are only other 2 types of link styles provided for auto layout: vertical and horizontal.

When the diagram becomes too complicate, the results are obviously unsatisfying. So, the effort presented in this paper targets at rearranging the blocks by putting the blocks in some new reasonable positions and to find optimal routes for the connection. After this automated process, the layout of a diagram should be enhanced and beautified for readability and maintainability.

\section{Technical Solution}

\subsection{Overview on Scilab/Xcos}

Scilab/Xcos palette provides varieties of predefined blocks such as signal processing, mathematical operations and discrete and continuous system blocks while it is also possible to develop user-defined blocks. Despite of different types of blocks, when it comes to representation, they all belong to BasicBlock. We can abstract an $\mathrm{X} \cos$ diagram as shown in Figure 2.

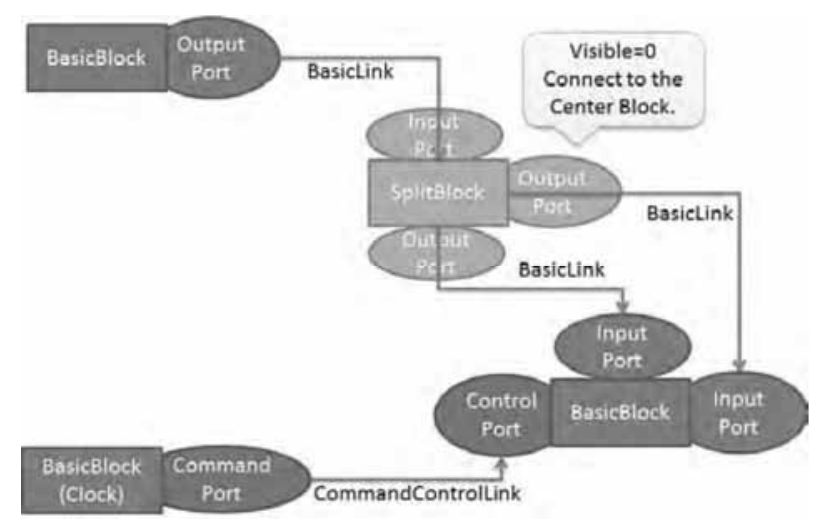

Figure 2: Basic Structure of Xcos Diagram. 
Normally, every block from palette is a BasicBlock. Every block owns its port(s) of input or output as its children (BasicPort) which belongs to four types: InputPort (subclasses: ExplicitInputPort or ImplicitInputPort) or OutputPort (subclass: ExplicitOutputPort or ImplicitOutputPort), ControlPort or CommandPort. Ports can be connected with links (BasicLink). ExplicitLink/ImplicitLink can be used to connect Input-Port and OutPutPort, and CommandControlLink can only be used to connect CommandPort and Control-Port. And there are also other classes implementing other functionality such as graph, palette and utilities.

All the Java codes of the Xcos program are in the 'org.scilab.modules.xcos' package. The structure is shown in Figure 3.

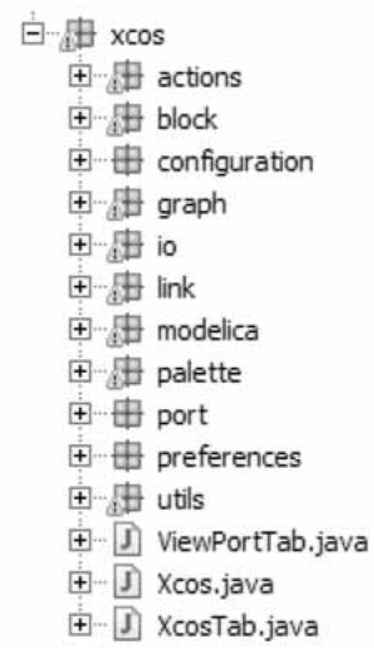

Figure 3: Structure of Xcos Java program.

Scilab/Xcos code base consists of various types of files, such as C codes, Java codes, xml files for help documents, image resources and files for locales. The user interface is generated by using Java. So, in order to implement an automatic layouting for Xcos, we need to conduct the implementation in Java.

\subsection{JGraphX}

JGraphX [12] is the underlying graphics framework of Scilab/Xcos. Aligned with that, the implementation of the technical solution encompasses utilization of JGraphX for the development of autmatic layouting features. JGraphX is a Java Swing diagramming (graph visualization) library licensed under the BSD license. The library is strong and easy to extend and inherit.
The documentation and Application Programming Interface (API) is quite mature. JGraphX not only provides functionality for visualization and interaction with node-edge graphs, but also includes functionality like XML support which would help save the current layout of the diagram avoiding that the layout needs to be recalculated every time it is opened. Besides the features about graph interaction and graph layouts which are being used in Xcos, JGraphX provides an analysis package which includes a range of analysis functions which provided us with a number efficient building blocks for the automatic layouting.

The core architecture of JGraphX includes the JGraphX model, the transactional model and mxCell. The JGraphX model (mxGraph) is the core model that describes the structure of the graph. The class called mxGraphModel is the underlying object that stores the data structure of the graph [12]. The graph class ScilabGraph in Scilab extends mxGraph. The transactional model is a transaction of models update which contains a series of actions. Transaction starts with beginUpdate and ends with endUpdate. With the help of transactional model, a set of events for the compound changes could be fired together after transaction. The mxCell is the cell object for both vertices and edges [12]. The three key attributes for an mxCell is its value, its style and its geometry. The ScilabGraphUniqueObject extends mxCell and it is also the ancestor of BasicBlock and BasicLink.

We use the geometry to change the position of blocks and use the style to change the routes of links in $\mathrm{Xcos}$. And we need to save all the states of Xcos diagram including the positions of blocks and the styles of links so that we do not need to re-calculate the layout every time re-opening an Xcos file.

\section{Optimal Link Style}

Optimal Link Style (OLS) is to find a route with more blank padding and with less turning and to use it as the style of a link.

\subsection{The Functional Flow}

The design of the functional flow can be briefed as below:

1. Change the style of the selected links one by one in a loop in one transaction. 
2. Check whether the two points of the ports are aligned and make sure that there are no blocks between them. If so, make the link with straight style, e.g. connect them directly.

3. Otherwise, use two new points each of which is a distance away from its corresponding port (if it was SplitBlock, use its center directly instead of its port).

4. Then start with these two new points, try to find the new route with one single turning point or 2 turning points. Otherwise, get another new point away from the starting point and use this to find a route with the same method. This could be re-cursed in several times.

5. Remove the unnecessary points and get the final optimal route for the link.

We modify the source files and create the classes listed in Table 1 to implement the functionality:

\begin{tabular}{ll}
\hline Class & Description \\
\hline StyleOptimalAction & Action events \\
\hline XcosRoute & Compute route \\
\hline XcosRouteUtils & Common utilities \\
\hline
\end{tabular}

Table 1: Classes Created.

\subsection{The Methods}

Here, we would like to introduce the methods and and underlying mathematical model for OLS. The method signatures are declared at the beginning of each section. Thus, the reader is informed about the application programming interface for that particular method.

\section{Get the position of a cell}

Method:

mxPoint getCenterPoint (mxICell cell, XcosDiagram graph)

This method is used to get the position of a cell where a link will connect to. There are three situations according to this cell:

- If it is a Port and its parent is a SplitBlock, use the center point of its parent.

- If it is a Port and its parent is not a SplitBlock, use the state of this cell (graph.getView().getState(cell)) to get port's mxCellState to get the point.

- If it is a BasicBlock, use the center point according to its geometry attribute.

\section{Check if a point is in a line segment}

Method:

boolean pointInLineSegment (double $x 1$, double y1, double x2, double y2, double x3, double y3)

This method is used to check $\mathrm{P}_{1}$ in Segment $\left(\mathrm{P}_{2}, \mathrm{P}_{3}\right)$ which is shown in Figure 4.

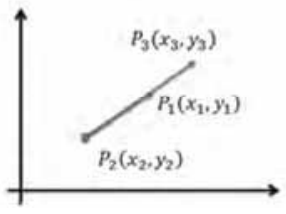

Figure 4: Point in Line Segment.

If it is, then $\mathrm{P}_{2} \mathrm{P}_{1}$ and $\mathrm{P}_{2} \mathrm{P}_{3}$ must have the same direction and $\mathrm{P}_{1}$ is between $\mathrm{P}_{2}$ and $\mathrm{P}_{3}$.

For the same direction:

$$
\begin{aligned}
\angle \overrightarrow{P_{2} P_{1}} & =\angle \overrightarrow{P_{2} P_{3}} \\
\frac{y_{1}-y_{2}}{x_{1}-x_{2}} & =\frac{y_{3}-y_{2}}{x_{3}-x_{2}} \\
\left(y_{1}-y_{2}\right)\left(x_{3}-x_{2}\right) & =\left(y_{3}-y_{2}\right)\left(x_{1}-x_{2}\right)
\end{aligned}
$$

For $\mathrm{P}_{1}$ between two points:

$$
\begin{aligned}
& \min \left(x_{2}, x_{3}\right) \leq x_{1} \leq \max \left(x_{2}, x_{3}\right) \\
& \min \left(y_{2}, y_{3}\right) \leq y_{1} \leq \max \left(y_{2}, y_{3}\right)
\end{aligned}
$$

\section{Check superimposition}

Method:

boolean linescoincide (double $\mathrm{x} 1$, double $\mathrm{y} 1$, double $x 2$, double y2, double x3, double y3, double $x 4$, double $y^{4}$ )

These two methods are used to check whether two lines coincide or not. The second one is to check strict superimposition of two line segments. In the first one, the lines would move parallel and then check all of them. This could avoid that two line segments are be too close.

Segment A $\left(\mathrm{P}_{1}, \mathrm{P}_{2}\right)$ and Segment B $\left(\mathrm{P}_{3}, \mathrm{P}_{4}\right)$ will coincide in these situations:

- Segment A is inside Segment B, e.g. both P1, and P2 are in Segment B;

- Segment B is inside Segment A, e.g. both P3, and P4 are in Segment B;

- Segment A and Segment B are parallel and one of the endpoints of one segment is in the other segment. 
If lines are parallel,

$$
\left(x_{1}-x_{2}\right)\left(y_{3}-y_{4}\right)=\left(x_{3}-x_{4}\right)\left(y_{1}-y_{2}\right)
$$

\section{Check obstacles}

Method:

boolean checkObstacle (double $\mathrm{x} 1$, double $\mathrm{y} 1$, double x2, double y2, Object[] allCells)

This method is used to detect whether there are obstacles between two points.

The definition of obstacles is: All top blocks and links and all the ports of blocks. EXCEPT: itself (link), its Source and Target (i.e. port) and SplitBlock.

If any of the below situations happens, it means that there is an obstacle between two points:

- If it is a Link,

- Check lines superimposition.

- Check whether points are in the link.

- If it is a Block,

- Use mxRectangle.intersectLine to get an intersection if it exists according to its geometry.

\section{Get orientation of ports}

Method:

Orientation getPortRelativeorientation

(BasicPort port, XcosDiagram graph) Orienta-

tion getNewOrien-tation(mxICell cell, double

cx, double cy, mxICell oth-ercell, double

ox, double oy, XcosDiagram graph)

These two methods are used to get the current orientation of a port according to its relative position to parent block.

We also consider the ports of different blocks, because the ports of a SplitBlock are not visible or the target point has no parent blocks.

- If its parent is a normal Block, calculate orientation according to the relative position of the port to its parent block as Figure 5 shows. For instance, the orientation of the port will be EAST if this port in the EAST zone of its parent block.

- If its parent is a SplitBlock, get the orientation of the InputPort of the SplitBlock according to the relative position of link's source (it is the same mathematical model in the first case); get the orientation of one OutputPort of the SplitBlock according to the orientation of the InputPort and the positions of both OutputPorts. For instance, when one of the OUT target is on NORTHEAST to IN source, its orientation will be
NORTH if the other out target is on its right side and the orientation of IN is not north; its orientation will be EAST if the other out target is on its left side.

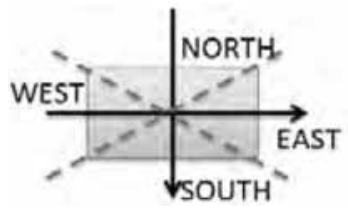

Figure 5: Orientation in Zones.

\section{Get a point away from port}

Method:

mxPoint getPointAwayPort (mxICell port, double portx, double porty, Orientation orien, Object [] allCells, XcosDiagram graph)

This method is used to get a new point away from a port according to the orientation of this Port as Figure 6 shows. If there are obstacles between the Port and the new point, reduce the distance and try another new point. Then use the new Point as the start/end point to compute the route.

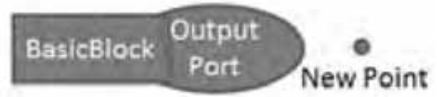

Figure 6: Get a New Point away from Port.

\section{Choose an optimal line}

Method:

double choosePoint (List<Double> list, double p1, double p2)

This method is used to choose a better line (which is the average number in the widest range in a certain density) from the discrete numbers as Figure 7 shows. Consider the points between $\mathrm{p} 1$ and $\mathrm{p} 2$ as a priority as Figure 8 shows.

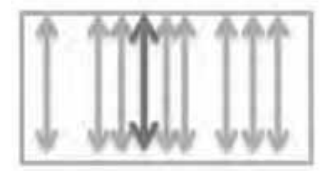

Figure 7: Choose an Optimal Line I.

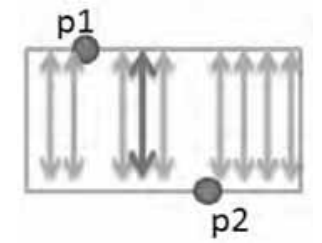

Figure 8: Choose an Optimal Line II. 


\subsection{Implementation in detail}

Firstly, we get the position of the source and the target cell. If two points are aligned (boolean isstrictlyAligned(double, double, double, double)) and there are no obstacles between them, then we connect two points directly and we do not need to do the steps further.

Then, we create a point away from the port according to the orientation of each port. Using these two new points as the new starting point and the ending point, find a simple route with 2 turning points. If the source is EAST/WEST orientation, we try the point $(x 2, y 1)$ as the turning point and check the obstacles among the new source point, this point and the new target point. In this case, the away point for the source is unnecessary. Otherwise, we try point $(\mathrm{x} 1, \mathrm{y} 2)$ and check the obstacles among the new source point, this point and the new target point. In this case, the away point for the source is necessary. The away point for the target is similar. This is shown in Figure 9. If the source is SOUTH/NORTH orientation, we try the point $(\mathrm{x} 1, \mathrm{y} 2)$ and check the obstacles among them. In this case, the away point for the source is necessary. Try the point $(\mathrm{x} 2, \mathrm{y} 1)$ and check the obstacles among them. In this case, the away point for the source is unnecessary. The away point for the target is also similar. This is shown in Figure 10. If we could get a route, we do not need to do the steps further.

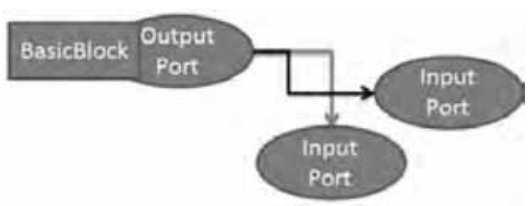

Figure 9: Single Turning I.

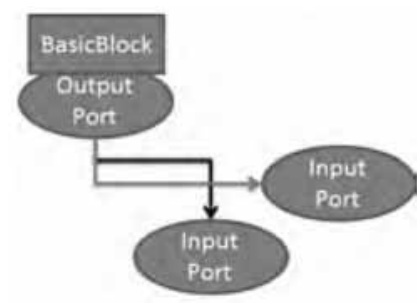

Figure 10: Single Turning II.

At the third step, we check all the possible horizontal or vertical connections of the points whose $\mathrm{y}$ or $\mathrm{x}$ is between the two points as Figure 11 shows ('possible' means no obstacles between points). In case that it is full of obstacles between two blocks, we extend the range of the detection.
If the orientation is horizontal, we check $\mathrm{x}$ firstly (the left one in the figure). Otherwise, check y firstly (the right one in the figure).

Finally, if there is no optimal solution in simple mode which is introduced above, we get new away points of the start point in 3 directions and use the new points to find a simple route. Otherwise, we try to find a complex route in a recursion.
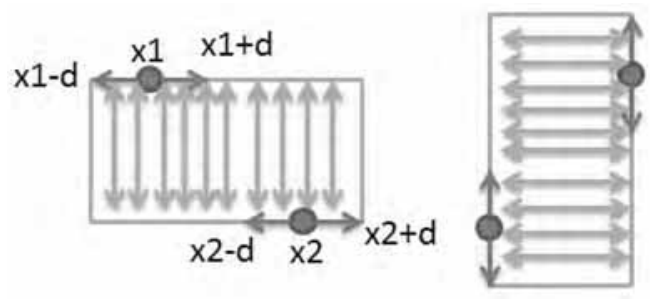

Figure 11: Simple Routes.

\subsection{A sample application}

In this example case, Figure 12 is the original diagram in a mess. Figure 13 is the diagram which we used OLS to format the links.

It looks better than previous layout and the links were clear for users to read. But it does not work well when there are split blocks. So we need to do some optimizations to make it more beautiful.

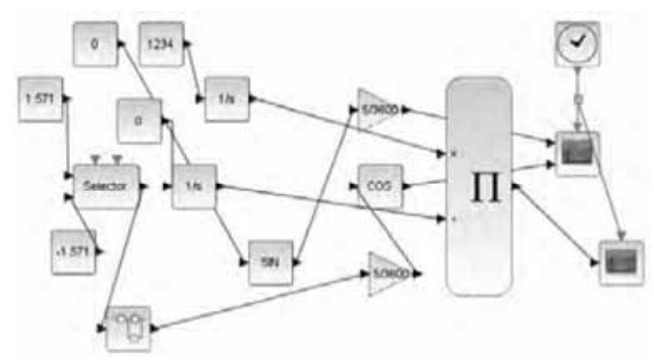

Figure 12: The Diagram in Original Version.

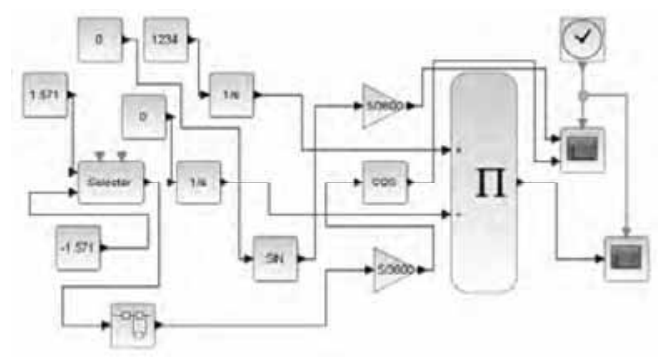

Figure 13: The Diagram after Using OLS. 


\section{Split Block Auto Position}

Split Block Auto Position (SBAP) is to find a position for split block where the links which connect to it look clearer in the optimal routes.

\subsection{The Functional Flow}

The design of functional flow can be introduced as below:

1. In the whole connection where the split block is, get one of the normal blocks as the source and all other blocks as the targets.

2. Compute their optimal routes separately.

3. Choose the conjunct point of the routes to be the new position of every split block.

4. Update the orientations of ports in the split block according to the routes.

5. After getting new position(s) and new orientations, update the links.

We modified the source files and created the classes listed in Table 2 to implement this functionality:

\begin{tabular}{ll}
\hline Class & Description \\
\hline AutoPositionSplitBlockAction & Action events \\
\hline BlockAutoPositonUtils & Calculate position \\
\hline
\end{tabular}

Table 2: Classes Created.

\subsection{The methods}

We will explain the methodology which is used for Split Block Auto Position. In this section, the methods and and underlying mathematical model for SBAP will be presented. As it was for the OSL, the method signatures are declared at the beginning of each subsection in order to reveal the application programming interface for that particular method.

\section{Get the root split block}

Method:

SplitBlock getRootSplitBlock (SplitBlock splitblock)

This method is used to get the root split block when there are multiple split blocks in the whole connection.

1. Check if the block which connects to the IN port of this split block is a normal block.
2. If it is a normal block, this split block is the root split block.

3. If it is a split block, then check as step 1 again and start this loop until find the normal block. Then the split block is the root one.

\section{Adjust routes}

Method:

void adjustRoutes (List<List<mxPoint»

listRoutes, object[] allobstacles,

List<mxICell> listPorts)

This method is used to adjust routes after getting the optimal routes (using OLS). As shown in Figure 14, some segments in two links might be parallel. We move segments to make them superimposed if there are no obstacles. Then there will be more superimpositions between routes and the last conjunct point of routes will be more meaningful.

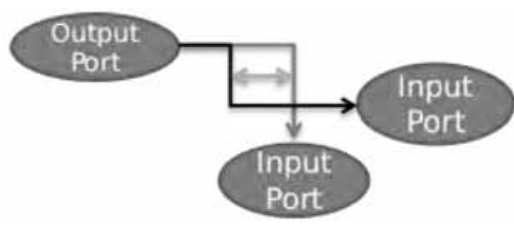

Figure 14: Adjusting Routes.

\section{Get the last conjunct point}

Method:

mxPoint getSplitPoint (List<List<mxPoint» listRoutes)

This method is used to get the last conjunct point of all routes. After the routes are adjusted, there are different last conjunct points between every 2 routes. We choose the one which is in all routes.

\section{Update orientation of port}

Method:

void updatePortorientation(SplitBlock split, List<List<mxPoint» listRoutes, XcosDiagram graph, BasicPort input)

Orientation get Inputorienttion (List<List

<mxPoint» list, mxPoint startPoint, mxPoint

splitPoint) Orientation getPortorienta-

tion(List<mxPoint> list, mxPoint splitPoint)

These methods are used to get the orientation of ports in a split block according to the relative routes and update them. There are routes passing the split block. As shown in Figure 15, 
1. For the IN port, from the split block point to the previous turning point is the orientation.

2. For the OUT port, there are 2 cases. If the split block is in the turning point, then from the turning point to next turning point is the orientation. Otherwise, from the previous turning point to the next turning point is the orientation.

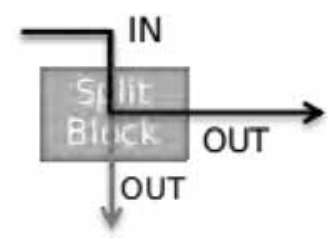

Figure 15: Get and Update Orientation.

\subsection{Implementation in detail}

When a link is split, there will be one split block generated. When the link is split several times, there will be several split blocks. Then one split block must have one IN port and two OUT ports.

1. Calculate the number of split blocks in this whole part of linking.

2. If there is only one split block,

- Get the port which connects to the IN port of the split block as a source. And get the ports which connect to the two OUT ports of the split block as targets.

- Find the optimal routes for the source to each target.

- Use the last conjunct point in both routes as the new position of split block as shown in Figure 16

- Find the orientations for each ports in the split block according to optimal routes.

3. If there are more than one split blocks,

- Get the root split block. Get the port which connects to the IN port of this root split block as a source.

- And get the ports of all basic blocks which connect to all other children split block as targets.

- Find the optimal routes for the source to each target.

- Use the last conjunct point in different routes as the new position of split blocks.

- Find the orientations for each ports in the split blocks according to optimal routes.

4. After getting new position(s) and new orientations, update the links.

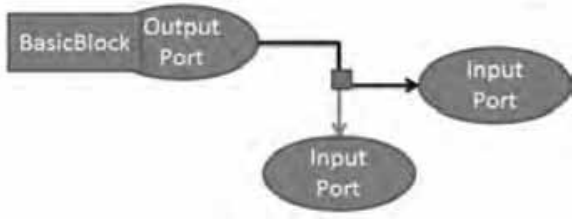

Figure 16: New Position of SplitBlock.

\subsection{A Sample Application}

In the case study, Figure 1 is the original diagram. Figure 17 is the diagram which we use SBAP and OLS to format.

Now, the layout is much better. The diagram is easy to read and maintain.

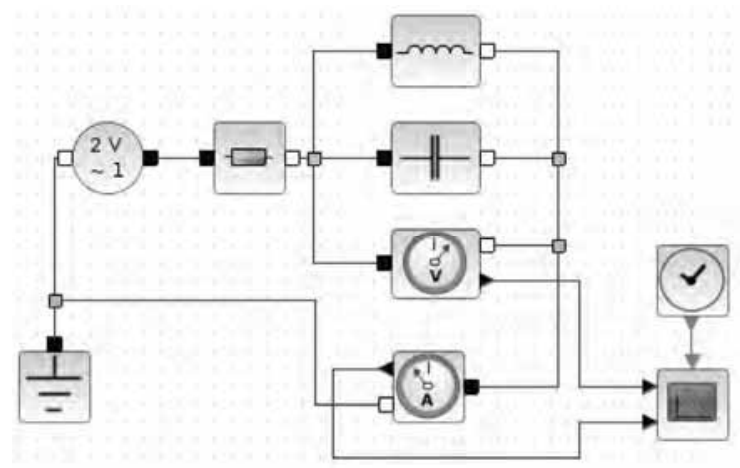

Figure 17: New Position of SplitBlock.

\section{Conclusion and Future Work}

The paper presents Optimal Link Style and Split Block Auto Position for automatic layouting Scilab/Xcos diagrams. Optimal Link Style focuses on the link styles which format the link, while Split Block Auto Position concentrates mainly on position changing of split blocks. According to his needs, user can decide in modeling time which one to apply.

Every project or every team has its own standards or criteria about the format of the diagrams. It is not easy to decide which layout is really perfect or fulfills the needs of users. What we have tried to achieve was not only providing an automatic layout, but also giving some options for users to make their own decisions about the final layout which they would like to maintain. On the other hand, user therefore needs to select the blocks or the links which he wants to change and click some buttons to make them in an optimal layout. That means that our automatic layout is still static instead of dynamic. And we could not get a preview of the automatic layout while we are drawing our diagrams or get a direct result about this automatic feature. 
Sometimes, users would like to draw a link with the optimal route when creating the connection between blocks. They would also like to format the layout of the links after they move some blocks without clicking some buttons.

Based on the previous paragraph, the future work includes improving the user experience. While the application programming interface for the layouting methods provides a baseline, it is necessary to experiment various user interaction scenarios.

\section{References}

[1] Champbell SL, Chancelier JP, Nikoukhah R. Modeling and Simulation in Scilab/Scicos. Springer Science and Business Media, Inc., 2006.

[2] Scilab Enterprise, Xcos Features. Retrieved July 30, 2016 from www.scilab.org/scilab/features/xcos.

[3] Frana P. An Interview with Edsger W. Dijkstra. Communications of the ACM 53 (8): 41-47, 2010.

[4] Dijkstra EW. A note on two problems in con nexion with graphs. Numerische Mathematik 1: 269-271, 1959.

[5] Bang-Jensen J, Gutin G. The Bellman- Ford-Moore algorithm. Digraphs: Theory, Algorithms and Applications, Springer Science and Business Media, 2000.
[6] Cormen TH, Leiserson CE, Rivest RL. Introduction to Algorithms (1st ed.). MIT Press and McGraw-Hill, 1990.

[7] Rosen KH. Discrete Mathematics and Its Applications, 5th Edition. Addison Wesley.2003.

[8] MathWorks, Simulation and Model-Based Design. Retrieved July 30, 2016 from http://www.mathworks.com/products/simulink/.

[9] Roulaeu G. Smart Signal Routing. Retrieved July 30, 2016 from http://blogs.mathworks.com/simulink/2012/10/11/smartsignal-routing/.

[10] Klauske LK, Dziobek C. Improving Modeling Usability: Automatic Layouting for Simulink. Retrieved July 30, 2016 from http://www.mathworks.com/videos/improvingmodeling-usability-automatic-layouting-for-simulink93139.html.

[11] Scilab Enterprise. Xcos for Very Beginners. Retrieved July 30, 2016 from http://www.scilab.org/community/news/20130830.

[12] JJGraph Ltd. GraphX (JGraph 6) User Manual. Retrieved July 30, 2016 from https://jgraph.github.io/. 



\title{
Mathematical Wave Fitting Models for the Quantification of the Diurnal Profile and Variability of Pulse Wave Analysis Parameters
}

\author{
Lena G. M. Bauer ${ }^{1 *}$, Bernhard Hametner², Christopher C. Mayer², S. Wassertheurer ${ }^{2}$ \\ ${ }^{1}$ Institute for Analysis and Scientific Computing, TU Wien, Wiedner Hauptstraße 8-10, \\ 1040 Vienna, Austria; ${ }^{*}$ lena.bauer@tuwien.ac.at \\ ${ }^{2}$ Center for Health \& Bioresources, AIT Austrian Institute of Technology, Biomedical Systems, Donau-City-Str. 1, \\ 1220 Vienna, Austria
}

SNE 27(3), 2017, 153-160, DOI: 10.11128/sne.27.tn.10386 Received: February 10, 2017, (Selected ASIM STS 2016 Postconf. Publ.), Accepted: February 25, 2017

SNE - Simulation Notes Europe, ARGESIM Publisher Vienna, ISSN Print 2305-9974, Online 2306-0271, www.sne-journal.org

Abstract. The analysis of 24 hour (24h) ambulatory blood pressure monitoring (ABPM) profiles and their variability has been of interest in literature for considerable time. The development of sophisticated algorithms, which are integrated into mobile sphygmomanometers, allows the performance of $24 \mathrm{~h}$ ABPM including pulse wave analysis (PWA). The recording involves the measurement of standard ABPM parameters as well as the estimation of central aortic pressures and other systemic cardiovascular parameters at regular time intervals throughout the day. The resulting time series often show a diurnal profile. Therefore, the analysis of these profiles and their variability is of interest. In this context, the analysis of diurnal blood pressure (BP) profiles serves as a model. The methods are adapted to be applicable to the time series independent of the parameter. In this article a selection of mathematical models and indices to quantify this profile and the variability of the time series are presented. The considered fitting models are a square wave fit, a fourier fit and a double logistic fit. The modelling process as well as advantages and disadvantages of each method are given. The results show that the algorithms performing the fits are feasible for the $24 \mathrm{~h}$ profiles and provide several indices quantifying certain characteristics of the profiles.

\section{Introduction}

Cardiovascular diseases are one of the leading causes for morbidity and mortality [1]. It is therefore of crucial importance to identify indicators for these diseases at an early stage to find proper treatment and prevent fatal outcome. There are many parameters describing the health condition of the cardiovascular system, the most popular being systolic and diastolic BP. However, hypertension is only able to predict $40 \%$ of coronary heart diseases [2]. Therefore, further indicators have to be found. The availability of oscillometric brachialcuff based blood pressure monitors, which include algorithms estimating central aortic pressures and other systemic cardiovascular parameters, enables the recording of ABPM and PWA parameters at regular time intervals throughout the day. The Mobil-O-Graph (I.E.M., Stolberg, Germany) is an example for such a monitoring device, which includes validated algorithms providing the PWA parameter values [3]. The resulting time series often show a diurnal profile. Therefore, the analysis of these profiles and their variability is of interest. In this context, the analysis of diurnal BP profiles serves as a model. These methods, which have been used in clinical studies for $24 \mathrm{~h}$ BP profiles for considerable time $[4,5,6]$, are adopted for other parameters of the PWA in order to mathematically quantify the variability of a time series regardless of the parameter. The aim of this article is to describe the calculation details of three such methods. All of them are curve fitting models which aim to assess the diurnal profile of the parameter time series. In general, this is achieved by an ansatz function of a specific form, which is fitted to the data set by a least squared error criterion. The advantages and disadvantages of each model are presented as well. The provided variability and profile indices might help to find further indicators for cardiovascular diseases.

\section{Methods}

This section deals with the motivation and calculation details of three fitting models: the square wave fit, the fourier fit and the double logistic fit. 
Least squared error criterion. Let $x_{1}, \ldots, x_{n}$ and $t_{1}, \ldots, t_{n}$ denote the measured values throughout the $24 \mathrm{~h}$ period and the corresponding time points, respectively. In general, the purpose of the curve fitting method is to determine the parameters $\lambda_{1}, \ldots, \lambda_{m}$ of an ansatz function $X_{\lambda_{1}, \ldots, \lambda_{m}}:[0,24) \longrightarrow \mathbb{R} \quad m<n$, which takes certain different forms as the parameters are varied, such that the residual sum of squares

$$
\sum_{i=1}^{n}\left(x_{i}-X_{\lambda_{1}, \ldots, \lambda_{m}}\left(t_{i}\right)\right)^{2}
$$

reaches its minimum.

\subsection{Square Wave Fit}

Motivation. BP tends to vary around a higher level during wakefulness than during night while being asleep in healthy patients $[5,7,8,9]$. The purpose of the square wave model is to capture this characteristic of the diurnal parameter profile. The period times of the higher and lower plateau are determined by the model.

Calculation. The ansatz function for the square wave model [4] is given by

$$
S W(t):= \begin{cases}a, & t \in\left\{t_{i}, t_{i+1}, \ldots, t_{i+k}\right\}, 1 \leq k<n \\ b, & t \in\left\{t_{1}, \ldots, t_{n}\right\} \backslash\left\{t_{i}, \ldots, t_{i+k}\right\},\end{cases}
$$

where $a$ and $b$ are the mean values of the data points $\left\{x_{i}, x_{i+1}, \ldots, x_{i+k}\right\}$ and of $\left\{x_{1}, \ldots, x_{n}\right\} \backslash\left\{x_{i}, \ldots, x_{i+k}\right\}$, respectively. The parameters $i$ and $k$ remain to be determined by the least squared error criterion. For a data set of $n$ measurements there exist $n \cdot(n-1)$ such square waves. In order to obtain the best fit curve with respect to the squared error, all possible square waves as well as the data points themselves are normalized. The data are transformed

$$
x_{1}, \ldots, x_{n} \longmapsto \tilde{x}_{1}, \ldots, \tilde{x}_{n}
$$

with

$$
\tilde{x_{i}}:=\frac{x_{i}-\bar{X}}{c S D} .
$$

The curve is transformed

$$
S W(t) \longmapsto S W_{s t}(t)
$$

with

$$
S W_{s t}(t):= \begin{cases}\frac{a-\overline{S W}}{\sigma_{S W}}, & t \in\left\{t_{i}, t_{i+1} \ldots, t_{i+k}\right\}, 1 \leq k<n \\ \frac{b-S W}{\sigma_{S W}}, & t \in\left\{t_{1}, \ldots, t_{n}\right\} \backslash\left\{t_{i}, \ldots, t_{i+k}\right\}\end{cases}
$$

and with

$$
\begin{gathered}
\overline{S W}=\frac{k \cdot a+(n-k) \cdot b}{n} \\
\sigma_{S W}^{2}=\frac{1}{n-1}\left(k \cdot(a-\overline{S W})^{2}+(n-k) \cdot(b-\overline{S W})^{2}\right) .
\end{gathered}
$$

For each of the standardized square waves the crosscorrelation coefficient is calculated as the average product of corresponding values of the curve and the original data, i.e.

$$
c c_{j}=\frac{1}{n} \sum_{i=1}^{n} S W_{s t}\left(t_{i}\right) \cdot \tilde{x}_{i} .
$$

These $n \cdot(n-1)$ values range from -1.0 to 1.0 , where a low value stands for a poor fit and 1.0 means that the curve is a perfect fit. Therefore, the curve with the highest cross-correlation value is chosen to be the best fit curve (Figure 2).

\subsection{Fourier Fit - Truncated Fourier Analysis}

Motivation. In this approach, a linear combination of cosine waves with different amplitudes and acrophases but known periods are fitted to the data. The motivation for this ansatz is Fouriers perception, that ,... any time series, regardless of its shape or regularity, can be described by a series of sine and cosine waves of various frequencies (Fourier 1822).'[10]

Calculation. The general ansatz in a fourier analysis is given by a fourier series

$$
F(t)=a_{0}+\sum_{k=1}^{\infty}\left(a_{k} \cdot \cos (k t)+b_{k} \cdot \sin (k t)\right)
$$

The model curve which is desired to describe the $24 \mathrm{~h}$ data profile is given by $[10,11]$

$$
\begin{gathered}
f(t):=M+C_{1} \cos \left(\frac{2 \pi t}{24}+\phi_{1}\right)+\cdots \\
\cdots+C_{k} \cos \left(\frac{2 \pi k t}{24}+\phi_{k}\right),
\end{gathered}
$$

where $M$ is called the mesor and $C_{1}, \ldots, C_{k}$ are constants representing the amplitudes of the cosine components. The acrophases (phase shifts, given in 
rad) are indicated by $\phi_{1}, \ldots, \phi_{k}$. As a first observation one sees that a finite number of ansatz functions instead of the infinite series is used (,truncated'). Further, it is sufficient to solely use cosine functions, since a sine function can always be replaced by a cosine function due to the relation $\sin (x)=\cos \left(x-\frac{\pi}{2}\right)$. Furthermore, all constants $C_{1}, \ldots, C_{k}$ can be assumed to be greater or equal to zero, since the sign of the cosine can be changes by a phase shift: $-\cos (x)=\cos (x-\pi)$. The period of the $i-$ th harmonic is equal to $\frac{24}{i}$ hours.

Using the addition theorem $\cos (A+B)=$ $\cos (A) \cos (B)-\sin (A) \sin (B)$ yields

$$
\begin{aligned}
f(t)= & M+C_{1} \cos \left(\frac{2 \pi t}{24}\right) \cos \left(\phi_{1}\right)+\cdots \\
& \cdots-C_{1} \sin \left(\frac{2 \pi t}{24}\right) \sin \left(\phi_{1}\right)+\cdots \\
& +\cdots \quad- \\
& \cdots+C_{k} \cos \left(\frac{2 \pi k t}{24}\right) \cos \left(\phi_{k}\right)+\cdots \\
& \cdots-C_{k} \sin \left(\frac{2 \pi k t}{24}\right) \sin \left(\phi_{k}\right) .
\end{aligned}
$$

The substitutions

$$
\begin{array}{ll}
X_{i}(t)=\cos \left(\frac{2 \pi i t}{24}\right), & a_{i}=C_{i} \cos \left(\phi_{i}\right) \\
Z_{i}(t)=\sin \left(\frac{2 \pi i t}{24}\right), & b_{i}=-C_{i} \sin \left(\phi_{i}\right)
\end{array}
$$

for $i=1, \ldots, k$ then lead to the linear regression model

$$
\begin{aligned}
f(t)=M & +a_{1} X_{1}(t)+b_{1} Z_{1}(t)+ \\
& +\cdots+ \\
& +a_{k} X_{k}(t)+b_{k} Z_{k}(t)
\end{aligned}
$$

The independent variables are here $X_{i}$ and $Z_{i}, i=$ $1, \ldots, k$. The variables $M, a_{i}$ and $b_{i}, i=1, \ldots, k$ have to be determined employing a (weighted) least squared error analysis. The (optional) weights are the lengths of the intervals between two consecutive measurements. The distance is seldom constant [12]. The values for $a_{i}$, $b_{i}$ und $M$ have to be determined in a way, that the residual sum of squares is minimal. In the following, the case is studied, where the sum of squared errors is extended by a weight $w_{i}$ for each data point $x_{i}$. Therefore, the following expression has to be minimized

$$
\begin{aligned}
R S S & =\sum_{i=1}^{n} w_{i}\left(x_{i}-f\left(t_{i}\right)\right)^{2} \\
& =\sum_{i=1}^{n} w_{i}\left(x_{i}-\left(M+\sum_{j=1}^{k}\left(a_{j} X_{j}\left(t_{i}\right)+b_{j} Z_{j}\left(t_{i}\right)\right)\right)\right)^{2} .
\end{aligned}
$$

If calculations should be done without any weighting, all $w_{i}$ can be set to one in the whole scheme. The above error estimate is minimal, if all the derivatives with respect to each parameter are equal to zero. Consider therefore

$\frac{\partial}{\partial M} R S S=\sum_{i=1}^{n} 2 \cdot w_{i}\left(x_{i}-\left(M+\sum_{j=1}^{k}\left(a_{j} X_{j}\left(t_{i}\right)+b_{j} Z_{j}\left(t_{i}\right)\right)\right)\right) \cdot(-1)$.

Setting this expression equal to zero and making the variables of interest, namely $a_{j}, b_{j}$ and $M$, explicit' leads to the first equation

$$
\sum_{i=1}^{n} w_{i} x_{i}=M \cdot \sum_{i=1}^{n} w_{i}+\sum_{j=1}^{k} a_{j}\left(\sum_{i=1}^{n} w_{i} X_{j}\left(t_{i}\right)\right)+\sum_{j=1}^{k} b_{j}\left(\sum_{i=1}^{n} w_{i} Z_{j}\left(t_{i}\right)\right)
$$

The derivatives with respect to the $a_{s}, 1 \leq s \leq k$ and $b_{s}, 1 \leq s \leq k$ yield to further $2 k$ equations. The total of $2 k+1$ equations can be written as a linear equation system in matrix form

$$
S \cdot \vec{l}=\vec{b},
$$

where $S$ is the matrix

$$
\left(\begin{array}{cccccccc}
w_{i} & w_{i} X_{1} & w_{i} X_{2} & \cdots & w_{i} X_{k} & w_{i} Z_{1} & \cdots & w_{i} Z_{k} \\
w_{i} X_{1} & w_{i} X_{1}^{2} & w_{i} X_{2} X_{1} & \cdots & w_{i} X_{k} X_{1} & w_{i} Z_{1} X_{1} & \cdots & w_{i} Z_{k} X_{1} \\
w_{i} X_{2} & w_{i} X_{1} X_{2} & w_{i} X_{2}^{2} & \cdots & w_{i} X_{k} X_{2} & w_{i} Z_{1} X_{2} & \cdots & w_{i} Z_{k} X_{2} \\
\vdots & \vdots & \vdots & \ddots & \vdots & \vdots & \vdots & \vdots \\
w_{i} X_{k} & w_{i} X_{1} X_{k} & w_{i} X_{2} X_{k} & \cdots & w_{i} X_{k}^{2} & w_{i} Z_{1} X_{k} & \cdots & w_{i} Z_{k} X_{k} \\
w_{i} Z_{1} & w_{i} X_{1} Z_{1} & w_{i} X_{2} Z_{1} & \cdots & w_{i} X_{k} Z_{1} & w_{i} Z_{1}^{2} & \cdots & w_{i} Z_{k} Z_{1} \\
\vdots & \vdots & \vdots & \vdots & \vdots & \vdots & \ddots & \vdots \\
w_{i} Z_{k} & w_{i} X_{1} Z_{k} & w_{i} X_{2} Z_{k} & \cdots & w_{i} X_{k} Z_{k} & w_{i} Z_{1} Z_{k} & \cdots & w_{i} Z_{k}^{2}
\end{array}\right),
$$

and in front of each entry of the matrix stands a sum $\sum_{i=1}^{n}$, and each $X$ and each $Z$ has $t_{i}$ as argument. Further,

$$
\vec{b}=\left(\begin{array}{c}
\sum_{i=1}^{n} w_{i} x_{i} \\
\sum_{i=1}^{n} w_{i} x_{i} X_{1}\left(t_{i}\right) \\
\vdots \\
\sum_{i=1}^{n} w_{i} x_{i} X_{k}\left(t_{i}\right) \\
\sum_{i=1}^{n} w_{i} x_{i} Z_{1}\left(t_{i}\right) \\
\vdots \\
\sum_{i=1}^{n} w_{i} x_{i} Z_{k}\left(t_{i}\right)
\end{array}\right) \text { and } \vec{l}=\left(\begin{array}{c}
M \\
a_{1} \\
\vdots \\
a_{k} \\
b_{1} \\
\vdots \\
b_{k}
\end{array}\right) \text {. }
$$


This linear equation system can be written as

$$
\left(X^{T} \cdot W \cdot X\right) \vec{l}=\left(X^{T} \cdot W\right) \vec{x},
$$

where $W=\operatorname{diag}\left(w_{1}, \ldots, w_{n}\right)$ is a diagonal matrix containing the weights, $\vec{x}=\left(x_{1}, \ldots, x_{n}\right)$ is the vector containing the given data and $X$ is the matrix

$X=\left(\begin{array}{ccccccc}1 & X_{1}\left(t_{1}\right) & \cdots & X_{k}\left(t_{1}\right) & Z_{1}\left(t_{1}\right) & \cdots & Z_{k}\left(t_{1}\right) \\ 1 & X_{1}\left(t_{2}\right) & \cdots & X_{k}\left(t_{2}\right) & Z_{1}\left(t_{2}\right) & \cdots & Z_{k}\left(t_{2}\right) \\ \vdots & \vdots & \vdots & \vdots & \vdots & \vdots & \vdots \\ 1 & X_{1}\left(t_{n}\right) & \cdots & X_{k}\left(t_{n}\right) & Z_{1}\left(t_{n}\right) & \cdots & Z_{k}\left(t_{n}\right)\end{array}\right)$.

This representation is simpler to implement. The solution is now given by

$$
\vec{l}=\left(X^{T} \cdot W \cdot X\right)^{-1} \cdot\left(X^{T} \cdot W\right) \vec{x} .
$$

Finally, the desired parameters of the model curve are calculated as

$$
\begin{gathered}
C_{i}=\sqrt{a_{i}^{2}+b_{i}^{2}} \\
\phi_{i}=\left\{\begin{array}{ll}
-\tan ^{-1}\left|\frac{b_{i}}{a_{i}}\right| & b_{i}>0 \wedge a_{i} \geq 0 \\
-\pi+\tan ^{-1}\left|\frac{b_{i}}{a_{i}}\right| & b_{i} \geq 0 \wedge a_{i}<0 \\
-\pi-\tan ^{-1}\left|\frac{b_{i}}{a_{i}}\right| & b_{i}<0 \wedge a_{i} \leq 0 \\
-2 \pi+\tan ^{-1}\left|\frac{b_{i}}{a_{i}}\right| & b_{i} \leq 0 \wedge a_{i}>0
\end{array} .\right.
\end{gathered}
$$

\subsection{Double Logistic Fit}

Motivation. Previously mentioned curve fitting methods partly work under at least one of two non legitimate assumptions.

- The parameter profile is perfectly symmetric. The assumption is that the decline of the parameter shows exactly the same characteristics as its surge (fourier fit, if only one harmonic is used and square wave fit).

- The periods, in which the considered parameter is higher respectively lower have the same length (fourier fit, if only one harmonic is used).

Both assumptions do not reflect reality - at least not for BP, for which the models were developed. The method of the double logistic analysis does not include any of these hypotheses. Head et al. developed this method for heart rate and BP data of rats [13]. In [14] they applied the method to heart data of humans.
Calculation. The model curve which is desired to describe the $24 \mathrm{~h}$ profile is given by $[13,14]$

$$
y(t)=P_{1}+\frac{P_{2}}{1+e^{P_{3}\left(P_{4}-t\right)}}+\frac{P_{2}}{1+e^{P_{5}\left(P_{6}-t\right)}},
$$

where $P_{1}$ to $P_{6}$ have to be determined. Such curves can be shaped as shown in Figure 1, depending on the choice for $P_{1}$ to $P_{6}$. The curve is then fitted to the data with a least squared error criterion.

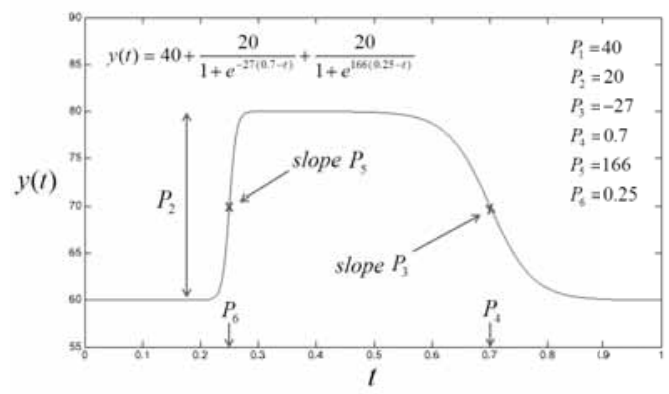

Figure 1: Example of a double logistic curve.

Implementation by Head et al. The model described in $[13,14]$ proceeds more complex as the authors add four terms to the model curve in equation 6 to obtain a quasi periodic function. These additional terms are related to the preceding and the following day. Another term $P_{2} \cdot q$ is added as a compensation parameter. The parameter $q$ is equal to -2 , if the data begin with the transition from high to low. Otherwise $q$ is chosen as 2 . The actual fitting curve therefore takes the form

$$
\begin{aligned}
y(t)=P_{1} & +\frac{P_{2}}{1+e^{P_{3}\left(P_{4}-t\right)}}+\frac{P_{2}}{1+e^{P_{5}\left(P_{6}-t\right)}} \\
& +\frac{P_{2}}{1+e^{P_{3}\left(P_{4}-t-24\right)}}+\frac{P_{2}}{1+e^{P_{5}\left(P_{6}-t-24\right)}} \\
& +\frac{P_{2}}{1+e^{P_{3}\left(P_{4}-t+24\right)}}+\frac{P_{2}}{1+e^{P_{5}\left(P_{6}-t+24\right)}} \\
& +P_{2} \cdot q .
\end{aligned}
$$

This double logistic ansatz function is then fitted by a specially developed computer program written in Labview. It makes use of the Marquardt algorithm, which optimizes the parameters by the least squared error criterion. This requires adequate start values for the variables $P_{1}$ to $P_{6}$. By iteration the parameters are optimized by minimizing the squared error. To obtain first approximations for these values, another fitting method, 
namely the Cosinor model (= fourier fit with one harmonic), is used. For instance, a first approximation for $P_{2}$ is taken as two times the amplitude of the cosinor fit. Furthermore, for the parameters several constraints are made. The limits for $P_{1}$ and $P_{2}$ are determined from the square wave fit. Mean values and standard deviations of the higher level period as well as of the lower level period, according to the square wave, are calculated. Define $y_{\max }$ as the mean of the higher level values plus two times the according standard deviation and $y_{\min }$ as the mean of the lower level values minus two times the according standard deviation. The constraints for $P_{1}$ and $P_{2}$ can then be chosen as

$$
\begin{aligned}
y_{\text {min }} \leq & P_{1}+P_{2}<y_{\text {max }} \\
& P_{2}>0 \\
y_{\text {min }} \leq & P_{1}+2 P_{2}<y_{\text {max }} .
\end{aligned}
$$

Constraints for the curvature parameters were chosen in a way that transition phases lasted for at least 30 minutes. Plateaus should be at least five hours long. Details to the algorithm can be found in $[13,14]$.

Implementation in MATLAB The approach to obtain a double logistic curve fit presented in this section is a simplified version of the one described above. It is done by the use of two different MATLAB built-in functions, namely nlinfit and lsqcurvefit, which fit the function given in formula 6 to the data set by the least squared error criterion. These two functions require start values for the parameters $P_{1}$ to $P_{6}$. They are obtained from the cosinor fit. The slopes at the two inflection points and their according time points are the initial values for $P_{3}$ to $P_{6}$. The level difference $P_{2}$ is chosen as the difference between the high level and the low level period as determined according to the cosinor method. $P_{1}$ is approximated by the difference of the low level mean and the approximation of $P_{2}$.

\section{Results}

Each of the described methods provides several indices quantifying the data profile. They are described in this section and exemplary plots of fitted curves are given.

\subsection{Square Wave Fit}

As can be seen in Figure 2, the square wave provides several indices quantifying the characteristics of the di- urnal profile of the data including the period durations of the higher and the lower plateau as well as the transition time points and the level difference.

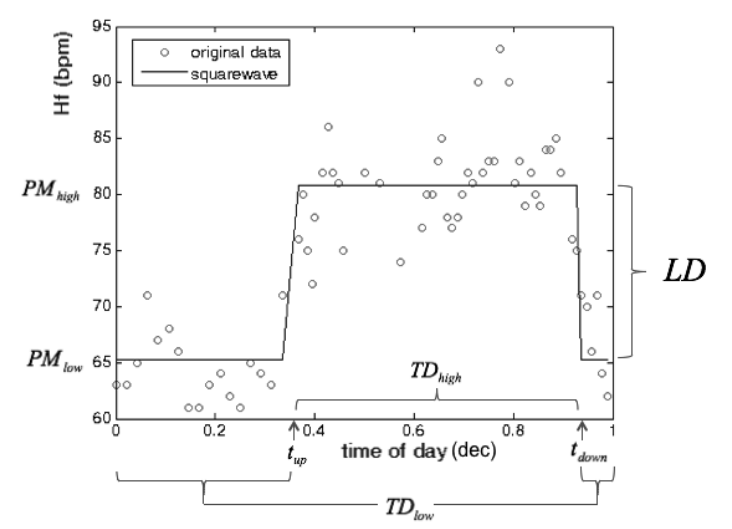

Figure 2: Square wave fitted to a data set of $24 \mathrm{~h}$ heart frequency $(\mathrm{Hf})$ data. The determined parameters $t_{i}=t_{u p}$ and $t_{i+k}=t_{d o w n}$ indicate the time points of the transition from the lower plateau to the higher plateau and vice versa. Further, the mean values in the periods $P M_{\text {high }}$ and $P M_{\text {low }}$, the period durations $T D_{\text {high }}$ and $T D_{\text {low }}$ as well as the level difference $L D$ are shown.

\subsection{Fourier Fit}

Basically the model provides two indices [12], which are graphically shown in Figure 3. As can be seen, the model predicts the occurrence of the maximum value at about 4 p.m., which is very close to the actual maximum. The overall amplitude serves as a measure for the range of the data.

\subsection{Double Logistic Fit}

The approach presented in the paragraph Implementation in MATLAB often yields favourable results for both of the functions nlinfit and lsqcurvefit as can be seen in Figure 4 at peripheral systolic BP as well as Hf data. However, for some data sets the curve is shaped unfamiliarly (Figure 8).

The indices obtained from the model are precisely the parameters $P_{1}$ to $P_{6}$ of the ansatz function. The parameters $P_{1}$ to $P_{6}$ represent the following qualities.

- $P_{1}+P_{2} \ldots$,baseline', , night - time - plateau'; This value is approximately the mean of the data measured during the lower level period. 


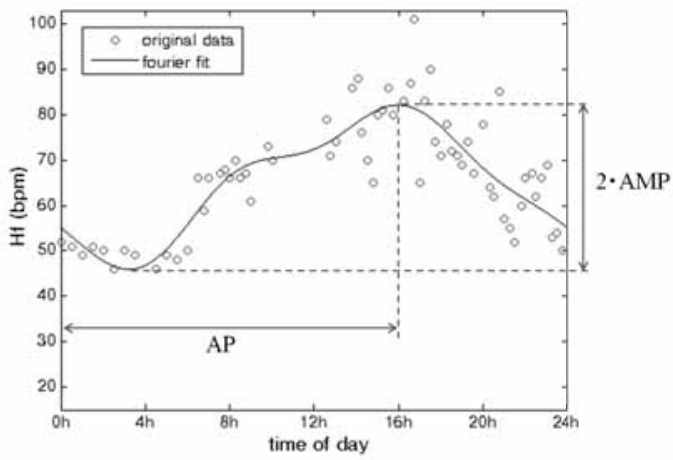

Figure 3: Fourier fit for $\mathrm{Hf}$ data using three harmonics. The parameters of the model are the overall acrophase (AP), which is defined as the time point of the maximal value of the model curve, and the amplitude (AMP), which is defined as half the extent of the range of the data.
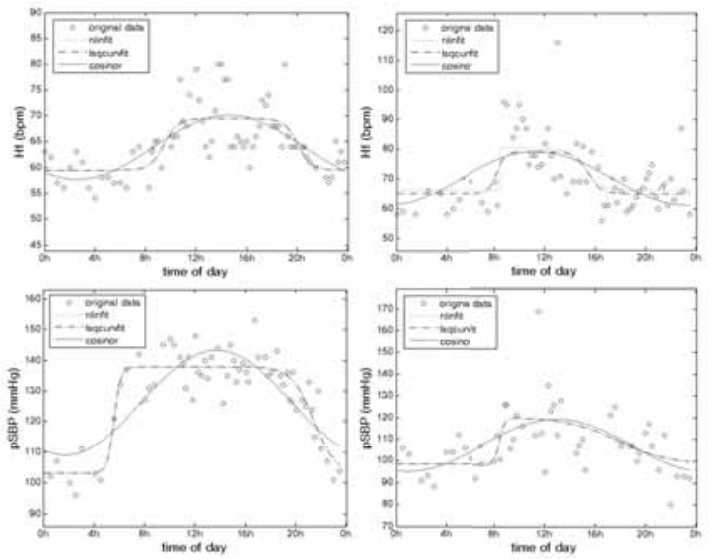

Figure 4: The double logistic functions take reasonable forms for different data sets ( $\mathrm{Hf}$ and pSBP). For the data in the top right corner, nlinfit and lsqcurvefit provide different curves. Nevertheless, both seem comprehensible.

- $P_{2} \ldots$,amplitude'; This represents the range of the data, the difference between the lower level and the higher level period, respectively.

- Accordingly, $P_{1}$ is the lower level value minus the difference of the two plateaus. Therefore, to obtain the approximation of the mean value of the high level period, one has to add the difference of the plateaus $P_{2}$ to the lower level plateau $P_{1}+P_{2}$, which equals $P_{1}+2 P_{2}$.
- $P_{3}$ and $P_{5}$ serve the modelling of the transitions between the plateaus. They indicate the extent of steepness of the change between the levels. While $P_{3}$ is the slope from the higher to the lower plateau, $P_{5}$ gives the slope of the reverse transition.

- The values $P_{4}$ and $P_{6}$ are the time points at which $50 \%$ of the transition is reached. Therefore, they are the middle time points within the transition periods.

Features of the MATLAB algorithm. One of the observations when applying the algorithm described in the section Implementation in MATLAB to different data sets is, that the curve does rise to the higher level but fails to fully return to the lower level plateau as can be seen in Figure 5. To avoid this unfavourable effect, two approaches can be made.
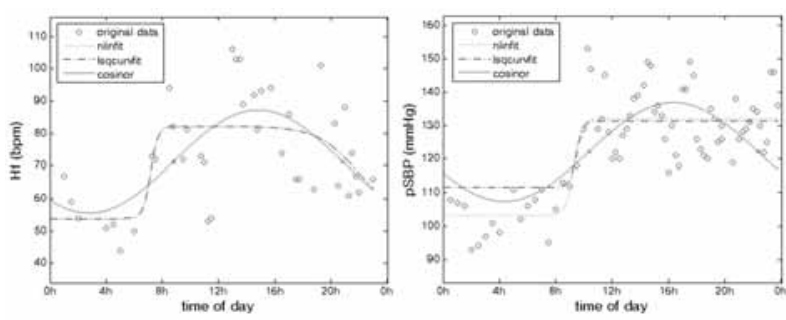

Figure 5: The plots show the unfavourable effect, that the double logistic curve does not return to the lower level plateau.

Since the start of the sleep time lies approximately within the interval $(22 \mathrm{~h}, 2 \mathrm{~h}),(\mathrm{BP})$ values begin to fall rather close to the end of the $24 \mathrm{~h}$ monitoring period. This might hinder the curve to perceive another low level period. To obtain enough lower values, the data set may be extended by a certain number of measurements of the following day. In the absence of these measurements, simply the first couple of hours of the same day with the according measurements are added. Applying the implemented MATLAB function on the data set with an extension of six hours to the same data sets as in Figure 5 leads to the desired return to the lower level plateau. This can be seen in Figure 6 .

The second option is to shift the time point of the beginning of the measurements such that transition periods are most likely not close to the beginning or the end of the observation period. 

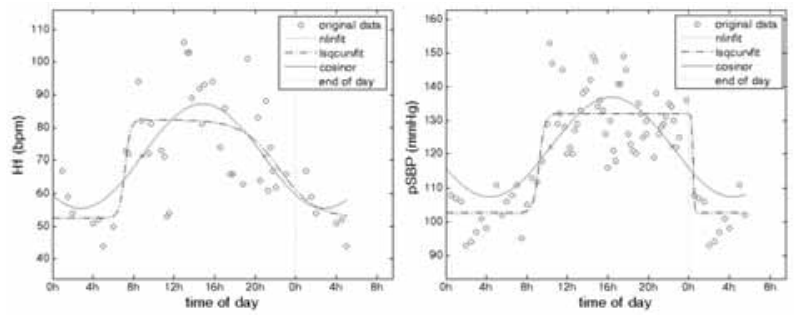

Figure 6: Double logistic curve fit with an extension of six hours to the data set.

Applying the MATLAB algorithm again to the same data sets as in Figure 5 and 6, respectively, with the start time set to 4 p.m. yields to the double logistic curves depicted in Figure 7. However, the shape of the curve is rather sensitive to the starting time, since the fitted functions in 6 and 7 show - at least for the data set on the left - notably different characteristics.
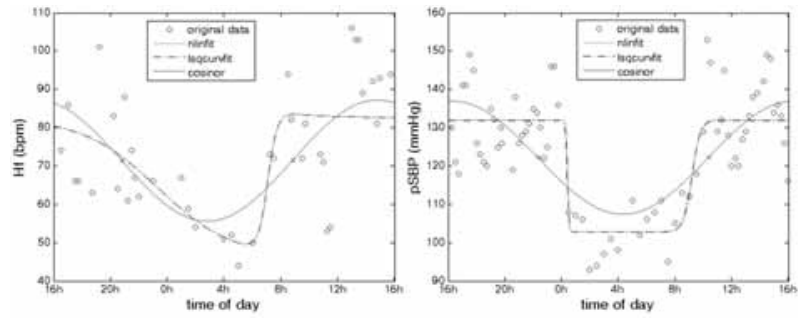

Figure 7: Double logistic curve fit with a shift of the starting time of the observations to 4 p.m.

\section{Discussion}

The advantages and disadvantages of each method are discussed in this section.

\subsection{Square Wave Fit}

This method of capturing the profile of the dataset is a refinement to the so called nocturnal BP fall [12]. There, the averaging of the data points in the alleged higher and lower periods is done over defined day time and night time periods which includes a subjective component. The square wave is advanced in the sense of correctness, since it is a method based on a mathematical model and the periods are implicitly determined [4]. In [15] it is further stated that the square wave approach performs better in fitting BP data as well as the heart rate changes than the Cosinor method.

Although the square wave captures several features of the parameter profile, while the degrees of freedom are limited to the two time points, when the level changes [4], one drawback of this approach is that the ansatz assumes abrupt and symmetrical transition periods. This does not reflect the fact that these transitions vary strongly from subject to subject - at least for BP data - [15].

\subsection{Fourier Fit}

There is no distinct statement which number of harmonics is the best choice. It is conjectured that various numbers of harmonics are possible 'best choices', depending on the (temporal) distance between two measurements [4]. Other authors hold that the model is better the more harmonics are used [11]. However, their recommended number is four harmonics, since the method performed best for different data sets and the influence of added harmonics on the indices of the model were negligible. As well as the square wave also the fourier analysis can be used to segment the $24 \mathrm{~h}$ interval in a lower level and a higher level period. However, Idema et al. [4] claim that the square wave method performs better considering segmentation.

The Fourier method captures the complexity of the signal better than the previously mentioned square wave approach. However, the smoothing effect might lead to an 'over-modelling' of the measurements [15]. Another advantage of this method is that it is applicable to nonequidistant data sets. Additionally, this method does not assume symmetrical period transitions or equal period durations [11].

\subsection{Double Logistic Fit}

The method is said to improve the modelling of the surge of (BP) data in the morning, which is known to be a risk factor for stroke $[13,16]$. The crucial innovation of this method is the possibility to consider the decline of the values and the rise separately [14]. Additionally, the model refrains from symmetry assumptions on the data profile. However, despite the presented favourable results, some further observations have to be mentioned. The implementation of this method by Head et al. $[13,14]$ is rather complex and the design of the curve in general seems to be only applicable to data sets with a specific shape. This can be seen in Figure 8. 
The data sets do not show a typical diurnal BP tenor, which leads to a rather unfamiliar double logistic fit. Head at al. include a lot of restrictions on the parameters of the model. This inclusion might improve the simplified approach presented above. Another observation made when applying the MATLAB algorithms on the data sets is, that the resulting curve is rather sensitive to the initial values. The improvement of the calculation of adequate initial values presents another field of investigation to obtain a solid method.

As the two MATLAB function often provide different results for the data sets, they require further analysis to find distinct quality criteria for the decision in favour of one of them.
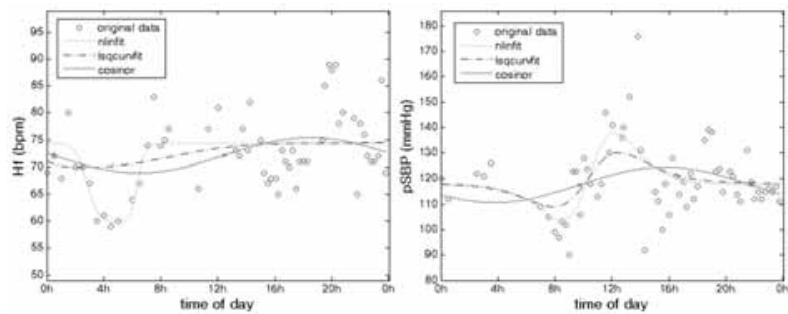

Figure 8: For some data sets the MATLAB algorithms yields to unfamiliar double logistic curves.

\section{Conclusion}

The results show that the algorithms performing the fits are feasible for the $24 \mathrm{~h}$ profiles and provide several indices quantifying certain characteristics of the profiles. Although the double logistic model requires further refinement, the results are encouraging.

\section{References}

[1] WHO-editor. Cardiovascular diseases Fact sheet Updated May 2017. website

http://www.who.int/mediacentre/ factsheets/fs317/en/visited on 09/07/2017.

[2] Gerstenblith G and Margolis S. Coronary heart disease. The John Hopkins White Papers, John Hopkins Medicine, Baltimore, Maryland. 2007

[3] Weber T, Wassertheurer S et al. Validation of a brachial cuff-based method for estimating central systolic blood pressure. Hypertension. 2011; 58(5): 825-832.
[4] Idema R N, Gelsema E S et al. A new model for diurnal blood pressure profiling - Square wave fit compared with conventional methods. Hypertension. 1992; 19(6): 595-605.

[5] Mancia G, Di Rienzo M et al. Ambulatory blood pressure monitoring use in hypertension research and clinical practice. Hypertension. 1993; 21(4): 510-524.

[6] Höcht C. Blood pressure variability: prognostic value and therapeutic implications. ISRN Hypertension. 2013; 2013. doi:

http://dx.doi.org/10.5402/2013/398485

[7] Degaute J-P, Van De Borne P et al. Quantitative analysis of the 24-hour blood pressure and heart rate patterns in young men. Hypertension. 1991; 18(2): 199-210.

[8] Millar-Craig M W, Bishop C N. Circadian variation of blood-pressure. The Lancet. 1978; 311(8068): 795-797.

[9] Sayk F, Becker C et al. To dip or not to dip. Hypertension. 2007; 49(5): 1070-1076.

[10] Refinetti R, Cornélissen G et al. Procedures for numerical analysis of circadian rhythms. Biological Rhythm Research. 2007; 38(4): 275-325.

[11] Thijs L, Staessen J A et al. Analysis of the diurnal blood pressure curve. High Blood Pressure and Cardiovascular Prevention. 1992; 1: 17-28.

[12] Thijs L, Staessen J A et al. Number of measurements required for the analysis of diurnal blood pressure profile. Journal of Human Hypertension. 1994; 8(4): 239-244.

[13] Head G A, Lukoshkova E V et al. Non-symmetrical double-logistic analysis of 24-h blood pressure recordings in normotensive and hypertensive rats. Journal of Hypertension. 2004; 22(11): 2075-2085.

[14] Head G A, Reid C M et al. Nonsymmetrical double logistic analysis of ambulatory blood pressure recordings. Journal of Applied Physiology. 2005; 98(4): 1511-1518.

[15] Parati G. Assessing circadian blood pressure and heart rate changes: advantages and limitations of different methods of mathematical modelling. Journal of Hypertension. 2004; 22(11): 2061-2064.

[16] Kario K, Pickering T G et al. Morning surge in blood pressure as a predictor of silent and clinical cerebrovascular disease in elderly hypertensives. Circulation. 2003; 107(10): 1401-1406. 


\title{
Brain Connectivity Models for Functional Magnetic Resonance Imaging
}

\author{
Nicole Geissberger ${ }^{1,2^{*}}$, Günter Schneckenreither ${ }^{2}$ \\ ${ }^{1}$ Center for Medical Physics and Biomedical Engineering, Medical University of Vienna, Währinger Gürtel 18-20, \\ 1090 Vienna, Austria; ${ }^{*}$ nicole.geissberger@tmsfmri.com \\ ${ }^{2}$ Institute for Analysis and Scientific Computing, TU Wien, Wiedner Hauptstraße 8-10, 1040 Vienna, Austria
}

SNE 27(3), 2017, 161-166, DOI: 10.11128/sne.27.tn.10387

Received: July 10, 2017; Revised: August 15, 2017;

Accepted: August 30, 2017

SNE - Simulation Notes Europe, ARGESIM Publisher Vienna, ISSN Print 2305-9974, Online 2306-0271, www.sne-journal.org

Abstract. When analyzing data from functional magnetic resonance imaging, different mathematical models are used. This article briefly describes the most important ones - starting with the so-called balloon model describing the hemodynamic response during brain activity, the General Linear Model widely used for functional localization of brain areas involved with certain stimuli, and Dynamic Causal Modelling as a framework for investigating dynamic brain connectivity models.

\section{Introduction}

Functional magnetic resonance imaging (fMRI) is a neuroimaging technique for investigating the inner mechanisms of the human brain during neuronal activity. A sequence of 3D images is acquired concurrently to stimuli to detect subtle changes in brain metabolism. Applications range from brain mapping - i.e. functional localization of brain regions associated with effects of interest - via research on brain connectivity to multimodal integration with other imaging and stimulation tools.

\section{Balloon Model}

The principle of fMRI is based on changes in cerebral blood supply. Neuronal activity causes an increased oxygen demand, therefore this demand must be met with an increase in inflow of highly oxygenated blood, overcompensating the energy demand.
Blood oxygenation increases, more specifically deoxygenated hemoglobin is reduced. Since deoxygenated hemoglobin $(\mathrm{dHb})$ is more paramagnetic than oxygenated hemoglobin $(\mathrm{oHb})$, magnetic field variations around $\mathrm{dHb}$ show reduced MR signal amplitudes. Thus, neuronal activity leads to a local decrease in $\mathrm{dHb}$ - causing an increased MR signal [5], [8] with a certain delay of several seconds.

This non-linear behaviour of the MR signal due to changes in blood supply is called the Blood Oxygenation Level Dependent (BOLD) effect. This is modelled using the so-called Balloon Model, linking changes in blood oxygenation caused by neuronal activity to the stimulus-induced hemodynamic response.
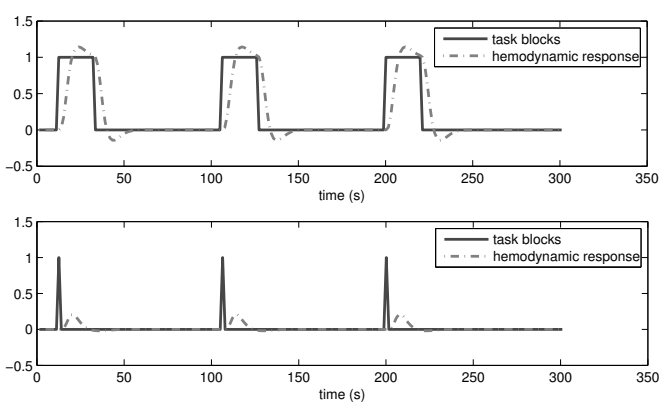

Figure 1: Hemodynamic response function (HRF) corresponding to task blocks with duration of 20 seconds (above) or events (below). Data created using the SPM software package.

The Balloon Model can be described by the following four differential equations, describing the signal $s$, the inflow of blood $f_{\text {in }}$, blood volume $v$, and deoxyhemoglobin $q$. It is assumed that the flow inducing signal $s$ and the change in regional cerebral blood flow $f_{\text {in }}$ are linked linearly,

$$
\dot{f}_{\text {in }}=s
$$


The flow inducing signal is generated by neuronal response to the stimulus function $u(t)$,

$$
\dot{s}=\varepsilon u(t)-\kappa s-\gamma\left(f_{\text {in }}-1\right)
$$

with the parameter $\varepsilon$ describing the efficacy of the stimulus to signal increase, $\kappa$ and $\gamma$ being rate constants describing signal decay and autoregulatory feedback from blood flow. The venous blood volume is the difference between inflow $f_{\text {in }}$ and outflow $f_{\text {out }}$ within a certain time interval (transit time) $\tau$,

$$
\dot{v}=\frac{f_{\text {in }}-f_{\text {out }}}{\tau} .
$$

The blood vessels show higher outflow rate when distended, modelled using a single stiffness parameter $\alpha$ as

$$
f_{\text {out }}=v^{\frac{1}{\alpha}}
$$

This describes the dynamic flow-volume relationship based on the so-called Windkessel model (see [6]). The deoxyhemoglobin content $q$ is modelled as an effect of blood flow and volume change,

$$
\begin{aligned}
\dot{q} & =\left(f_{\text {in }} \frac{E\left(f_{\text {in }}, \rho\right)}{\rho}-f_{\text {out }}(v) \frac{q}{v}\right) \frac{1}{\tau}, \\
E\left(f_{\text {in }}, \rho\right) & =1-(1-\rho)^{1 / f_{\text {in }}},
\end{aligned}
$$

with $E\left(f_{\text {in }}, \rho\right)$ describing the oxygen extraction from the inflowing arterial blood, and $\rho$ being the constant oxygen extraction fraction.

The six unknown biophysical parameters are thus: the stimulus efficacy $\varepsilon$, the rate constant for signal decay $\kappa$, the rate constant for blood flow autoregulation $\gamma$, the stiffness parameter $\alpha$, the resting oxygen extraction fraction $\rho$ and the mean transit time $\tau$. Commonly used estimates for these parameters can be seen in [2].

Assuming these six parameters to be constant, this allows to view the model described by these differential equations as a single input single output model - the input being the stimulus function $u(t)$ and the output being the measured BOLD signal $x(t)$. It can be written as

$$
x(t)=H(u(t))
$$

with $H$ being the hemodynamic response function (HRF), translating the stimulus $u(t)$ into the measured signal change $x(t)$. In practice, the time courses of the observed data and stimulus function are represented as vectors, thus can be written as

$$
\mathbf{x}=H(\mathbf{u})
$$

\section{General Linear Model (GLM)}

The General Linear Model is one of the most versatile models used in statistics. Many common statistical approaches, such as analysis of variance (ANOVA), ttests, ordinary linear regression etc. are special cases of this model [11].

For a series of measurements $\mathbf{y}$ and a matrix of explanatory variables - the so-called design matrix, the General Linear Model is described as

$$
\mathbf{y}=\mathbf{X} \beta+\varepsilon .
$$

Here, $\beta$ describes the parameters for modelling the linear coherence between the influencing factors and the output signal; $\varepsilon$ is the residual vector containing errors or noise, which are assumed independent and identically distributed.

\subsection{Embedding the Balloon Model in GLM}

Since for fMRI data the signal is not measured directly but rather through the hemodynamic response, this behaviour must be taken into account. The Balloon Model is incorporated into the design matrix as follows:

$$
\begin{aligned}
\mathbf{X} & =H(\mathbf{U}) \\
\mathbf{y} & =\mathbf{X} \beta+\varepsilon .
\end{aligned}
$$

For simplicity and because hemodynamic parameters can be assumed to be constant [2], the hemodynamic response function is usually not mentioned explicitly. The transformation of the stimulus functions in the design matrix is obtained by convoluting the stimulus function with the HRF.

\subsection{Application}

In functional MRI data 3D images are taken at every time point, resulting in a time course for each voxel. The coefficients $\beta$ are estimated minimizing the error squares. This Least Squares estimation can be easily performed using the so-called pseudoinverse of the design matrix:

$$
\beta \approx\left(\mathbf{X}^{\top} \mathbf{X}\right)^{-1} \mathbf{X}^{\top} \mathbf{y}
$$


To test for significance of the estimated $\beta$, the residual term $\mathbf{r}$ needs to be examined. It is defined as the difference between the measured values and the estimation,

$$
\mathbf{r}=\mathbf{y}-\mathbf{X} \beta .
$$

The standard deviation $\hat{\sigma}$ of the error term is estimated as

$$
\hat{\sigma}=\frac{\mathbf{r}^{\top} \cdot \mathbf{r}}{v}
$$

where $v$ is the number of degrees of freedom associated with $\mathbf{r}$. For independently distributed residuals in fMRI, this would be the number of scans minus the number of effects estimated (column rank of $\mathbf{X}$ ). The standard error $e$ can be described as

$$
e^{2}=\mathbf{c} \cdot \hat{\sigma}\left(\mathbf{X}^{\top} \mathbf{X}\right)^{-1} \cdot \mathbf{c}^{\top} .
$$

Now the t-score is obtained as

$$
t=\frac{\mathbf{c} \cdot \beta}{e} .
$$

The General Linear Model is estimated separately for each voxel, resulting in a 3D map of parameter estimates and corresponding t-scores which can be used to functionally localize brain activity associated with certain stimuli or tasks.

The described methods for functional neuroimaging data are implemented in the SPM package for MATLAB, which was developed by the Wellcome Trust Centre for Neuroimaging at the University College London. The software, as well as documentation and course files, is freely available at http://www.fil.ion.ucl. ac.uk/spm/software/.

\subsection{Criticism}

The GLM gives a robust and easy-to-use method for fMRI analyses, as no prior knowledge of function or activation maps are required to do a standard GLM analysis. However, it is limited to regarding just snapshots of brain states, neglecting brain dynamics and interplay of different cortical regions. Therefore, other methods must be used for more complex questions concerning interconnections and co-dependencies of brain regions.

\section{Dynamic Causal Modelling (DCM)}

Dynamic Causal Modelling is a framework which can be used for investigating hypotheses on effective brain connectivity, modelling the network behaviour of certain well-defined brain regions. This is done using following differential equation,

$$
\dot{\mathbf{z}}=F\left(\mathbf{z}, u, \theta_{c}\right)=\left(\mathbf{A}+\sum_{j} u_{j} \mathbf{B}^{j}\right) \mathbf{z}+\mathbf{C} u
$$

where $\mathbf{z}$ is the state vector describing the activity in a number of neuronal populations, $u$ is the (timedependent) input, the parameter $\theta_{c}$ describes the (timeindependent) coupling parameters, consisting of following adjacency matrices.

Here $\mathbf{A}$ describes the static influence of activity in each neuronal population on others independent of conditions (task/input-independent effective connectivity), $\mathbf{B}^{j}$ describes the condition-dependent intermodulations for each condition $j$, and $\mathbf{C}$ describes the direct influence of the conditions on activity in each brain region. The indices of the matrix entries specify the direction of the modulations - the column index indicating the source and the row index indicating the target of the connection.

Thus, the modeller needs to decide which regions of interest should be included in the model, as well as which connections are hypothesized to be present or not. The connection strength (i.e. the values of the entries in the connectivity matrices) is subsequently estimated from the data.

An example of such structural hypotheses in a DCM with 3 neuronal populations is depicted in figure 2 . Here, the assumed static connectivity is shown in blue, non-existing connections are indicated in red, influence of the conditions are shown in green. This DCM is translated into following $\mathbf{A}, \mathbf{B}$ and $\mathbf{C}$ matrices:

$$
\begin{array}{r}
{\left[\begin{array}{c}
\dot{z_{1}} \\
\dot{z_{2}} \\
\dot{z_{3}}
\end{array}\right]=\left(\left[\begin{array}{ccc}
a_{1,1} & a_{1,2} & 0 \\
a_{2,1} & a_{2,2} & a_{2,3} \\
0 & a_{3,2} & a_{3,3}
\end{array}\right]+u_{1}\left[\begin{array}{ccc}
0 & b_{1,2} & 0 \\
0 & 0 & 0 \\
0 & 0 & 0
\end{array}\right]\right)\left[\begin{array}{l}
z_{1} \\
z_{2} \\
z_{3}
\end{array}\right]} \\
+\left[\begin{array}{cc}
a_{1} & 0 \\
0 & 0 \\
0 & 0
\end{array}\right]\left[\begin{array}{l}
u_{1} \\
u_{2}
\end{array}\right]
\end{array}
$$




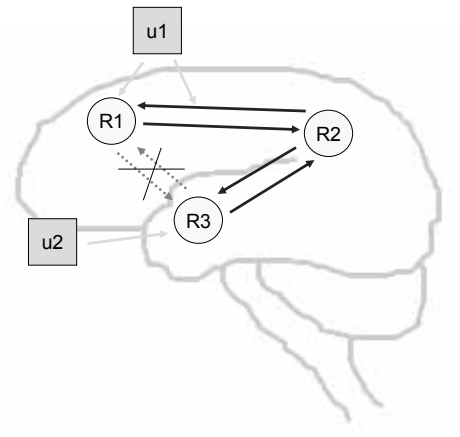

Figure 2: Schematic image of DCM structural assumptions with 3 neuronal populations.

\subsection{Embedding the Balloon Model in DCM}

As before, neuronal activity is not measured directly but via the BOLD effect - thus the hemodynamic state equations in section 1 need to be incorporated into the model as well. This leads to following full model from stimulus $u(t)$ to measured signal $y(t)$ :

stimulus functions

$$
\begin{gathered}
u(t) \\
\downarrow \\
\text { neural state equation } \\
\frac{d x}{d t}=\left(A+\sum_{j=1}^{m} u_{j} B^{j}\right) x+C u \\
\downarrow
\end{gathered}
$$

hemodynamic state equations

$$
\begin{gathered}
\dot{s}=x-\kappa s-\gamma(f-1) \\
\dot{f}=s \\
\tau \dot{v}=f_{\text {in }}-v^{\frac{1}{\alpha}} \\
\tau \dot{q}=f_{\text {in }} \frac{E\left(f_{\text {in }}, \rho\right)}{\rho}-v^{\frac{1}{\alpha}} \frac{q}{v} \\
\downarrow
\end{gathered}
$$

BOLD signal change equation

$$
y=\lambda(v, q)
$$

The BOLD signal change is modelled as a function of blood volume $v$ and deoxyhemoglobin content $q$,

$$
\lambda(v, q)=V_{0}\left(k_{1}(1-q)+k_{2}(1-q / v)+k_{3}(1-v)\right)
$$

taking into account the blood volume fraction $V_{0}$ and constants $k_{1}, k_{2}$ and $k_{3}$. These constants have been estimated by Buxton et al. [1] for a field strength of 1.5 Tesla. The variables of interest lying within the neural state equation, they can be estimated using a model inversion framework.

\subsection{Application}

Current implementations of DCM [4] within the SPM package use a Bayesian framework for model estimation, iteratively updating the parameters based on prior estimates to maximize the model evidence $p(y \mid m)$,

$$
p(y \mid m)=\int p(y \mid \theta, m) p(\theta \mid m) d \theta
$$

where $y$ is the measured data and $m$ is the model.

Maximizing the model evidence. As the model evidence (likelihood) can not be evaluated analytically, it needs to be approximated. Usually the maximization task is performed based on the log-likelihood due to numerical advantages when reaching very low values (for $p(y \mid m) \ll 1)$.

To reduce the risk of overfitting, state-of-the-art methods for model evidence estimations, such as the Bayesian Information Criterion (BIC) or the Akaike Information Criterion (AIC), generally use an accuracy and a complexity term to find the best balance between model fit and complexity.

$$
\log p(y \mid m) \approx \operatorname{accuracy}(m)-\operatorname{complexity}(m) .
$$

These criteria are defined as

$$
\begin{array}{r}
-\mathrm{AIC}=\log p(y \mid \theta, m)-n \\
-\mathrm{BIC}=\log p(y \mid \theta, m)-\frac{n}{2} \log (N)
\end{array}
$$

where $y$ stands for the data, $m$ is the model with parameters $\theta, N$ is the number of data points and $n$ is the number of parameters. Here, the complexity term penalizes a high number of parameters, however not their information content. Therefore, the so-called negative free energy was introduced,

$$
F=\langle\log p(y \mid \theta, m)\rangle_{q}-\mathrm{KL}[q(\boldsymbol{\theta}), p(\boldsymbol{\theta} \mid m)]
$$


where $q$ is the assumed conditional distribution, thus the left term is the expected log-likelihood under this posterior. KL denotes the Kullberg-Leibler divergence, which takes into account not only the number of parameters, but also their co-dependencies and therefore information gain,

$$
\begin{array}{r}
\mathrm{KL}[q(\theta), p(\theta \mid m)]=\frac{1}{2}\left(\log \left|C_{\theta}\right|-\log \left|C_{\theta \mid y}\right|\right. \\
\left.+\left(\mu_{\theta \mid y}-\mu_{\theta}\right)^{T} C_{\theta}^{-1}\left(\mu_{\theta \mid y}-\mu_{\theta}\right)\right) .
\end{array}
$$

Here, $C_{\theta}$ is the empirical prior covariance, $C_{\theta \mid y}$ is the posterior covariance, $\mu_{\theta}$ and $\mu_{\theta \mid y}$ are the prior and posterior expectation. The negative free energy is a lower bound for the log-likelihood and can thus be used as its approximation for parameter estimation and making single subject and group inferences on model structure.

Note that these measures - AIC, BIC as well as negative free energy - are relative measures only. Thus, they can be used to compare relative fit of different models, however not for testing overall model quality in the sense of hypothesis testing.

Parameter estimation. In the Dynamic Causal Modelling framework, both biophysical $\left(\theta_{h}=(\varepsilon, \kappa, \gamma, \alpha, \rho, \tau)\right.$, see section 1$)$ and connectivity parameters $\left(\theta_{c}=(A, B, C)\right)$ need to be estimated. As a non-linear optimization problem, current implementations of DCM use a Gauss-Newton algorithm in an Expectation Maximization Scheme (see [4]) to calculate the maximum a-posteriori (MAP) estimates. Here, a fully Bayesian approach is used, based on the conditional probability of the parameters given the data, $p(\theta \mid y)$.

According to Bayes' theorem, following proportionality is given:

$$
p(\theta \mid y) \propto p(y \mid \theta) p(\theta)
$$

Under Gaussian assumptions for the posterior density $p(\theta \mid y)$, its estimation is reduced to finding its first two moments - the conditional mean $\eta_{\theta \mid y}$ and covariance $C_{\theta \mid y}$ of $\theta \mid y$. Analogously, the priors are estimated in terms of their expectation $\eta_{\theta}$ and $C_{\theta}$.

From equation (24) we receive

$$
p(\theta \mid y) \propto \exp \left[-\frac{1}{2}\left(\theta-\eta_{\theta \mid y}^{(i+1)}\right)^{\top} C_{\theta \mid y}^{-1}\left(\theta-\eta_{\theta \mid y}^{(i+1)}\right)\right]
$$

with

$$
\begin{aligned}
C_{\theta \mid y} & =\left(\bar{J}^{\top} \bar{C}_{e}^{-1} \bar{J}\right)^{-1}, \\
\eta_{\theta \mid y}^{(i+1)} & =\eta_{\theta \mid y}^{(i)}+C_{\theta \mid y}\left(\bar{J}^{\top} \bar{C}_{e}^{-1} \bar{J}\right)
\end{aligned}
$$

where

$$
\bar{y}=\left[\begin{array}{c}
y-h\left(\eta_{\theta \mid y}^{(i)}\right) \\
\eta_{\theta}-\eta_{\theta \mid y}^{(i)}
\end{array}\right], \bar{J}=\left[\begin{array}{l}
J \\
1
\end{array}\right], \bar{C}_{e}=\left[\begin{array}{cc}
C_{e} & 0 \\
0 & C_{\theta}
\end{array}\right] .
$$

Iterating equation (25) when no priors are given can be seen as the Gauss-Newton method for parameter estimation (see [3] for further details).

Although we have so far assumed the error covariance $C_{e}$ to be known, usually it is not, e.g. for temporal correlations in fMRI. However, it can be estimated using some hyperparameters $\lambda_{j}$, so that

$$
\begin{aligned}
C_{e} & =\sum_{j} \lambda_{j} Q_{j} \quad \text { and } \\
Q_{j} & =\frac{\partial C_{e}}{\partial \lambda_{j}} .
\end{aligned}
$$

$Q_{j}$ represents a covariance basis set that embody variance components, which can model different variances for different data blocks, or even temporal correlations within blocks. To model the error covariance using this basis set, we must now estimate its coefficients $\lambda_{j}$.

The posterior mean $\eta_{\theta \mid y}$ and covariance $C_{\theta \mid y}$ as well as error covariance $C_{e}$ are now estimated iteratively in an expectation maximization (EM) scheme, such that the model evidence - i.e. the negative free energy $F-$ is maximized. See [3] and [7] for more details.

Inference strategies. After specification and estimation of a DCM, for each connectivity parameter in $\theta_{c}$ there exists an estimate of the mean and covariance, as well as an estimate of the model evidence through the negative free energy $F$.

On the parameter level, the significance of each connection can be tested using one-sided t-tests on the mean and covariance - analogously to section 2.2.

However, as the negative free energy is only a relative measure of model quality, we can not infer on the model itself, but only compare different models (model structures) on the same data, giving following options for inferences on the model structure level:

- Select the model maximizing the model evidence for each subject. 
- Average over models showing high posterior probability.

The first option is also referred to as Bayesian Model Selection (BMS). As there are often several models showing non-neglectable posterior probability, the second option has become an often preferred choice - referred to as Bayesian Model Averaging (BMA) - performing a weighted average over models within an Occam's window, i.e. showing a posterior probability above a certain threshold, see [9] and [10].

\subsection{Criticism}

Compared to classic GLM approaches, DCM inherently takes into account the dynamic nature of the brain by implementing interconnections between brain regions. However, the so-called "effective connectivity" investigated in the DCM does not imply direct physical connection of certain brain regions.

Also, while in the GLM analysis no prior assumptions have to be made on spatial extent and locations of activation changes, DCM depends strongly on a-priori hypotheses regarding neuronal populations and mechanisms.

Exploratory, i.e. non-hypothesis-driven analysis, could theoretically be conducted by setting each measurement point, i.e. voxel time course, in relation to each other and variating the existing connections respectively. However, this is not only numerically impossible, but also the winning model might not be optimal.

Within the current DCM framework it is only possible to estimate the most plausible parameters for predefined modulatory connections, but it is not possible to validate the prior assumptions (i.e. presumed connections) per se, and thus the resulting model. Thus, the DCM framework can only be regarded as a model selection framework. Other, possibly invasive, methods must be used for finding further evidence on the validity of the models.

\section{Acknowledgement}

We thank Christian Windischberger and his team at the Medical University of Vienna for sharing their knowledge and experience throughout this work.

\section{References}

[1] Buxton RB, Wong EC, and Frank, LR. Dynamics of blood flow and oxygenation changes during brain activation: the balloon model. Magnetic Resonance in Medicine. 1998; 39(6):855-864.

[2] Friston KJ, Mechelli A, Turner R, and Price CJ. Nonlinear responses in fMRI: the balloon model, volterra kernels, and other hemodynamics. NeuroImage. 2000; 12(4):466-477.

[3] Friston KJ. Bayesian estimation of dynamical systems: an application to fMRI. NeuroImage. 2002; 16(2):513-530.

[4] Friston K, Harrison L, and Penny W. Dynamic Causal Modelling. NeuroImage. 2003; 19:1273-1302.

[5] Logothetis NK, Pausl J, Augath M, Trinath T, and Oeltermann A. Neurophysiological investigation of the basis of the fMRI signal. Nature. 2001; 412(6843):150-157.

[6] Mandeville JB, Marota JJ, Ayata C, Zaharchuk G, Moskowitz MA, Rosen BR, and Weiskoff RM. Evidence of a cerebrovascular postarteriole windkessel with delayed compliance. Journal of Cerebral Blood Flow \& Metabolism. 1999; 19(6):679-689.

[7] Neal RM and Hinton GE. A view of the EM algorithm that justifies incremental, sparse, and other variants. In: Jordan MI. Learning in Graphical Models. First edition. Dordrecht: Springer Netherlands; 1998. pp 355-368.

[8] Ogawa S, Lee T-M, Kay AR, and Tank DW. Brain magnetic resonance imaging with contrast dependent on blood oxygenation. Proceedings of the National Academy of Sciences. 1990; 87(24):9868-9872.

[9] Penny WD, Stephan KE, Mechelli A and Friston KJ. Comparing dynamic causal models. NeuroImage. 2004; 22(3):1157-1172.

[10] Penny WD, Stephan KE, Daunizeau J, Rosa MJ, Friston KJ, Schofield TM and Leff AP. Comparing families of dynamic causal models. PLoS Comput Biol. 2010; 6(3):e1000709.

[11] Sachs L. Angewandte Statistik: Anwendung Statistischer Methoden. Eighth edition. Berlin Heidelberg: Springer-Verlag; 1997. 885 p. 


\section{SNE Simulation News \\ EUROSIM Data and Quick Info}
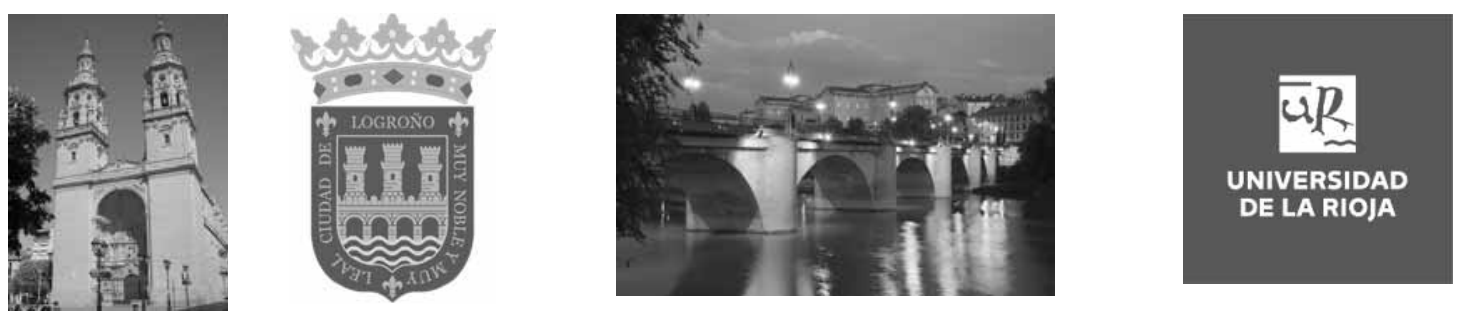

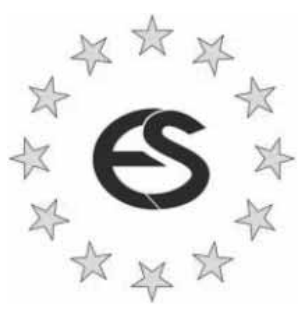

Contents

Short Info EUROSIM N2

Short Info ASIM, CEA-SM SG ...N3

Short Info CROSSIM , CSSS, DBSS, FRANCOSIM ............N4

Short Info HSS, ISCS, LIOPHANT, LSS ...........................N5

Short Info KA-SIM , PSCS, SIM S ..................................N6

Short Info SLOSIM , RNSS, UKSIM ................................N7

Short Info ROM SIM , M IM OS, Albanian Soc. ................ 8

Simulation Notes Europe SNE is the official membership journal of EUROSIM and distributed / available to members of the EUROSIM Societies as part of the membership benefits.

If you have any information, announcement, etc. you want to see published, please contact a member of the editorial board in your country or the editorial office. For scientific publications, please contact the EiC.

This EUROSIM Data \& Quick Info compiles data from EUROSIM societies and groups: addresses, weblinks, and officers of societies with function and email, to be published regularly in SNE issues.

This information is also published at EUROSIM's website www.eurosim.info.

\section{SNE Reports Editorial Board}

EUROSIM Emilio Jiménez, emilio.jimenez@unirioja.es Andreas Körner, andreas.koerner@tuwien.ac.at Miguel Mujica Mota, m.mujica.mota@hva.nl

ASIM A. Körner, andreas.koerner@tuwien.ac.at CEA-SMSG Emilio Jiménez, emilio.jimenez@unirioja.es CROSSIM Vesna Dušak,vdusak@foi.hr CSSS Mikuláš Alexík, alexik@frtk.utc.sk DBSS M. Mujica Mota, m.mujica.mota@hva.nl FRANCOSIM Karim Djouani, djouani@u-pec.fr HSS András Jávor, javor@eik.bme.hu ISCS M. Savastano, mario.savastano@unina.it LIOPHANT F. Longo,f.longo@unical.it LSS Yuri Merkuryev, merkur@itl.rtu.lv PSCS Zenon Sosnowski, zenon@ii.pb.bialystok.pl RNSS Y. Senichenkov, senyb@dcn.icc.spbstu.ru SIMS Esko Juuso,esko.juuso@oulu.fi SLOSIMVito Logar,vito.logar@fe.uni-lj.si UKSIM A. Orsoni, A.Orsoni@kingston.ac.uk KA-SIM Edmond Hajrizi, info@ka-sim.com MIM OS Paolo Proietti, roma@mimos.it ROMSIM Marius Radulescu, mradulescu@ici.ro Albanian Society Kozeta Sevrani, kozeta.sevrani@unitir.edu.al SNE Editorial Office / ARGESIM

$\rightarrow$ www.sne-journal.org, www.eurosim.info

䒠- office@sne-journal.org, Andreas Körner, (info, news)

豐eic@sne-journal.org, Felix Breitenecker (publications)

$\triangle$ SNE Editorial Office, Andreas Körner c/ o ARGESIM / M athematical M odelling $\&$ Simulation Group, TU Wien /101,

Wiedner Hauptstrasse 8-10, 1040 Vienna , Austria 


\section{EUROSIM}

\section{$S$ Federation of European Simulation Societies}

General Information. EUROSIM, the Federation of European Simulation Societies, was set up in 1989. The purpose of EUROSIM is to provide a European forum for simulation societies and groups to promote advancement of modelling and simulation in industry, research, and development. $\rightarrow$ www.eurosim.info

Member Societies. EUROSIM members may be national simulation societies and regional or international societies and groups dealing with modelling and simulation. At present EUROSIM has 16 Full Members and 2 Observer Members, and one member candidate.

\begin{tabular}{|c|c|}
\hline ASIM & $\begin{array}{l}\text { Arbeitsgemeinschaft Simulation } \\
\text { Austria, Germany, Switzerland }\end{array}$ \\
\hline CEA-SMSG & $\begin{array}{l}\text { Spanish M odelling and Simulation Group } \\
\text { Spain }\end{array}$ \\
\hline CROSSIM & $\begin{array}{l}\text { Croatian Society for Simulation M odeling } \\
\text { Croatia }\end{array}$ \\
\hline CSSS & $\begin{array}{l}\text { Czech and Slovak Simulation Society } \\
\text { Czech Republic, Slovak Republic }\end{array}$ \\
\hline DBSS & $\begin{array}{l}\text { Dutch Benelux Simulation Society } \\
\text { Belgium, Netherlands }\end{array}$ \\
\hline FRANCOSIM & $\begin{array}{l}\text { Société Francophone de Simulation } \\
\text { Belgium, France }\end{array}$ \\
\hline HSS & Hungarian Simulation Society; Hungary \\
\hline ISCS & $\begin{array}{l}\text { Italian Society for Computer Simulation } \\
\text { Italy }\end{array}$ \\
\hline KA-SIM & Kosovo Simulation Society, Kosovo \\
\hline LIOPHANT & $\begin{array}{l}\text { LIOPHANT Simulation Club } \\
\text { Italy \& International }\end{array}$ \\
\hline LSS & Latvian Simulation Society; Latvia \\
\hline PSCS & $\begin{array}{l}\text { Polish Society for Computer Simulation } \\
\text { Poland }\end{array}$ \\
\hline MIMOS & $\begin{array}{l}\text { Italian M odelling and Simulation } \\
\text { Association, Italy, Observer M ember }\end{array}$ \\
\hline RNSS & $\begin{array}{l}\text { Russian National Simulation Society } \\
\text { Russian Federation }\end{array}$ \\
\hline ROMSIM & $\begin{array}{l}\text { Romanian Society for M odelling and Sim- } \\
\text { ulation, Romania, Observer M ember }\end{array}$ \\
\hline SIMS & $\begin{array}{l}\text { Simulation Society of Scandinavia } \\
\text { Denmark, Finland, Norway, Sweden }\end{array}$ \\
\hline SLOSIM & $\begin{array}{l}\text { Slovenian Simulation Society } \\
\text { Slovenia }\end{array}$ \\
\hline UKSIM & $\begin{array}{l}\text { United Kingdom Simulation Society } \\
\text { UK, Ireland }\end{array}$ \\
\hline
\end{tabular}

EUROSIM Board / Officers. EUROSIM is governed by a board consisting of one representative of each member society, president and past president, and representatives for SNE Simulation Notes Europe. The President is nominated by the society organising the next EUROSIM Congress. Secretary, Secretary to the Board, and Treasurer are elected out of members of the board.

\begin{tabular}{ll}
\hline President & $\begin{array}{l}\text { Emilio Jiménez (CAE-SM SG), } \\
\text { emilio.jimenez@ unirioja.es }\end{array}$ \\
\hline Past President & $\begin{array}{l}\text { Esko Juuso (SIM S) } \\
\text { esko.juuso@ oulu.fi }\end{array}$ \\
\hline Secretary & $\begin{array}{l}\text { M. Mujica M ota (DBSS), } \\
\text { m.mujica.mota@ hva.nl }\end{array}$ \\
\hline Treasurer & $\begin{array}{l}\text { Felix Breitenecker (ASIM ) } \\
\text { felix.breitenecker@ tuwien.ac.at }\end{array}$ \\
\hline $\begin{array}{c}\text { Secretary to the } \\
\text { Board }\end{array}$ & $\begin{array}{l}\text { Andreas Körner } \\
\text { andreas.koerner@tuwien.ac.at }\end{array}$ \\
\hline SNE & Felix Breitenecker \\
Representative & felix.breitenecker@ tuwien.ac.at \\
\hline
\end{tabular}

SNE - Simulation Notes Europe. SNE is a scientific journal with reviewed contributions as well as a membership newsletter for EUROSIM with information from the societies in the News Section. EUROSIM societies are offered to distribute to their members the journal SNE as official membership journal. SNE Publishers are EUROSIM, ARGESIM and ASIM.

\begin{tabular}{ll}
\hline $\begin{array}{l}\text { SNE } \\
\text { Editor-in-Chief }\end{array}$ & $\begin{array}{l}\text { Felix Breitenecker } \\
\text { felix.breitenecker@tuwien.ac.at }\end{array}$ \\
$\rightarrow$ www.sne-journal.org, \\
麦- office@sne-journal.org
\end{tabular}

EUROSIM Congress. EUROSIM is running the triennial conference series EUROSIM Congress. The congress is organised by one of the EUROSIM societies.

EUROSIM 2019, the $10^{\text {th }}$ EUROSIM Congress, will be organised by CAE-SMSG, the Spanish simulation society, in La Rioja, Logroño, Spain, in July 2019.

\begin{tabular}{l} 
Chairs / Team EUROSIM 2019 \\
\hline $\begin{array}{r}\text { Emilio Jiménez, EUROSIM President, } \\
\text { emilio.jimenez@unirioja.es }\end{array}$ \\
Juan Ignacio Latorre, juanignacio.latorre@unavarra.es \\
\hline www.eurosim.info
\end{tabular}




\section{EUROSIM Member Societies}

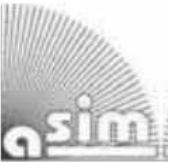

\section{ASIM \\ German Simulation Society \\ Arbeitsgemeinschaft Simulation}

ASIM (Arbeitsgemeinschaft Simulation) is the association for simulation in the German speaking area, servicing mainly Germany, Switzerland and Austria. ASIM was founded in 1981 and has now about 600 individual members, and 90 institutional or industrial members.

$$
\begin{aligned}
& \rightarrow \text { www.asim-gi.org with members' area } \\
& \text { 麦= info@asim-gi.org, admin@asim-gi.org } \\
& ₫ \text { ASIM - Inst. f. Analysis and Scientific Computing } \\
& \text { Vienna University of Technology (TU Wien) } \\
& \text { Wiedner Hauptstraße 8-10, } 1040 \text { Vienna, Austria }
\end{aligned}
$$

\begin{tabular}{|c|c|}
\hline \multicolumn{2}{|l|}{ ASIM Officers } \\
\hline President & $\begin{array}{l}\text { Felix Breitenecker } \\
\text { felix.breitenecker@tuwien.ac.at }\end{array}$ \\
\hline \multirow[t]{2}{*}{ Vice presidents } & Sigrid Wenzel, s.wenzel@uni-kassel.de \\
\hline & $\begin{array}{l}\text { T. Pawletta, } \\
\text { thorsten.pawletta@hs-wismar.de }\end{array}$ \\
\hline \multirow[t]{2}{*}{ Secretary } & Ch. Deatcu, christina.deatcu@ @s-wismar.de \\
\hline & A. Körner, andreas.koerner@tuwien.ac.at \\
\hline Treasurer & Anna M athe, anna.mathe@tuwien.ac.at \\
\hline \multirow{4}{*}{$\begin{array}{l}\text { Membership } \\
\text { Affairs }\end{array}$} & S. Wenzel, s.wenzel@uni-kassel.de \\
\hline & W. M aurer, werner.maurer@zhwin.ch \\
\hline & Ch. Deatcu, christina.deatcu@hs-wismar.de \\
\hline & F. Breitenecker, felix.breitenecker@tuwien.ac.at \\
\hline \multirow[t]{2}{*}{ Repr. EUROSIm } & F. Breitenecker, felix.breitenecker@tuwien.ac.at \\
\hline & A. Körner, andreas.koerner@tuwien.ac.at \\
\hline \multirow{2}{*}{$\begin{array}{l}\text { Int. Affairs - } \\
\text { GI Contact }\end{array}$} & N. Popper, niki.popper@drahtwarenhandlung.at \\
\hline & O. Rose, Oliver.Rose@tu-dresden.de \\
\hline \multirow[t]{2}{*}{$\begin{array}{l}\text { Editorial Board } \\
\text { SNE }\end{array}$} & $\begin{array}{l}\text { T. Pawletta, } \\
\text { thorsten.pawletta@hs-wismar.de }\end{array}$ \\
\hline & Ch. Deatcu, christina.deatcu@hs-wismar.de \\
\hline Web EuroSim & A. Körner, andreas.koerner@tuwien.ac.at \\
\hline
\end{tabular}

ASIM is organising / co-organising the following international conferences:

- ASIM Int. Conference 'Simulation in Production and Logistics' - bi-annual

- ASIM 'Symposium Simulation Technique' - biannual

- MATHMOD Int. Vienna Conference on Mathmatical Modelling - triennial

Furthermore, ASIM is co-sponsor of WSC - Winter Simulation Conference

\begin{tabular}{cl}
\hline ASIM Working Committee \\
\hline GM MS & $\begin{array}{l}\text { Methods in M odelling and Simulation } \\
\text { Th. Pawletta, thorsten.pawletta@hs-wismar.de }\end{array}$ \\
\hline SUG & $\begin{array}{l}\text { Simulation in Environmental Systems } \\
\text { J.Wittmann, wittmann@ informatik.uni- } \\
\text { hamburg.de }\end{array}$ \\
\hline STS & $\begin{array}{l}\text { Simulation of Technical Systems } \\
\text { Walter Comerell, Commerell@ hs-ulm.de }\end{array}$ \\
\hline SPL & $\begin{array}{l}\text { Simulation in Production and Logistics } \\
\text { Sigrid Wenzel, s.wenzel@ uni-kassel.de }\end{array}$ \\
\hline EDU & $\begin{array}{l}\text { Simulation in Education/Education in Simulation } \\
\text { A. Körner, andreas.koerner@tuwien.ac.at }\end{array}$ \\
\hline BIG DATA & $\begin{array}{l}\text { Working Group Data-driven Simulation in Life } \\
\text { Sciences; niki.popper@ dwh.at }\end{array}$ \\
\hline & $\begin{array}{l}\text { Working Groups for Simulation in Business } \\
\text { Administration, in Traffic Systems, for Standardi- } \\
\text { sation, etc. }\end{array}$ \\
\hline
\end{tabular}

\section{CEA-SM SG - Spanish Modelling and Simulation Group}

CEA is the Spanish Society on Automation and Control and it is the national member of IFAC (International Federation of Automatic Control) in Spain. Since 1968 CEA-IFAC looks after the development of the Automation in Spain, in its different issues: automatic control, robotics, SIMULATION, etc. The association is divided into national thematic groups, one of which is centered on Modeling, Simulation and Optimization, constituting the CEA Spanish Modeling and Simulation Group (CEA-SMSG). It looks after the development of the Modelling and Simulation (M\&S) in Spain, working basically on all the issues concerning the use of M\&S techniques as essential engineering tools for decisionmaking and optimization.

$\rightarrow$ http://www.ceautomatica.es/grupos/

$\rightarrow$ emilio.jimenez@unirioja.es

simulacion@cea-ifac.es

$\triangle$ CEA-SMSG / Emilio Jiménez, Department of Electrical Engineering, University of La Rioja, San José de Calasanz 31, 26004 Logroño (La Rioja), SPAIN

\begin{tabular}{ll}
\hline CEA - SM SG Officers \\
\hline President & $\begin{array}{l}\text { Emilio Jiménez, } \\
\text { emilio.jimenez@unirioja.es }\end{array}$ \\
\hline Vice president & $\begin{array}{l}\text { Juan Ignacio Latorre, } \\
\text { juanignacio.latorre@ unavarra.es }\end{array}$ \\
\hline Repr. EUROSIM & Emilio Jiménez, emilio.jimenez@ unirioja.es \\
\hline Edit. Board SNE & Emilio Jiménez, emilio.jimenez@ unirioja.es \\
\hline Web EURoSıM & Mercedes Perez mercedes.perez@ unirioja.es \\
\hline
\end{tabular}




\section{CROSSIM - Croatian Society for Simulation Modelling}

CROSSIM-Croatian Society for Simulation Modelling was founded in 1992 as a non-profit society with the goal to promote knowledge and use of simulation methods and techniques and development of education. CROSSIM is a full member of EUROSIM since 1997.

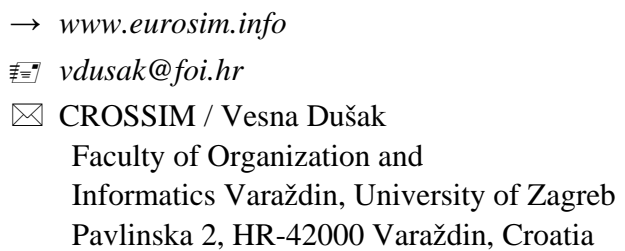

\begin{tabular}{ll}
\multicolumn{2}{l}{ CROSSIM Officers } \\
\hline President & Vesna Dušak, vdusak@foi.hr \\
\hline Vice president & Jadranka Božikov, jbozikov@ snz.hr \\
\hline Secretary & Vesna Bosilj-Vukšić, vbosilj@ efzg.hr \\
\hline Executive board & Vlatko Čerić, vceric@ efzg.hr \\
\cline { 2 - 2 } members & Tarzan Legović, legovic@irb.hr \\
\hline Repr. EuRoSım & Jadranka Božikov, jbozikov@ snz.hr \\
\hline Edit. Board SNE & Vesna Dušak, vdusak@foi.hr \\
\hline Web EuRoSım & Jadranka Bozikov, jbozikov@ snz.hr \\
\hline
\end{tabular}

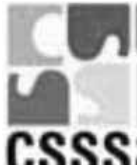

\section{CSSS - Czech and Slovak}

\section{Simulation Society}

CSSS -The Czech and Slovak Simulation Society has about 150 members working in Czech and Slovak national scientific and technical societies (Czech Society for Applied Cybernetics and Informatics, Slovak Society for Applied Cybernetics and Informatics). The main objectives of the society are: development of education and training in the field of modelling and simulation, organising professional workshops and conferences, disseminating information about modelling and simulation activities in Europe. Since 1992, CSSS is full member of EUROSIM.

$\rightarrow$ www.fit.vutbr.cz/CSSS

玤”snorek@fel.cvut.cz

$\bowtie$ CSSS / Miroslav Šnorek, CTU Prague

FEE, Dept. Computer Science and Engineering,

Karlovo nam. 13, 12135 Praha 2, Czech Republic

\begin{tabular}{ll}
\hline CSSS Officers & \\
\hline President & Miroslav Šnorek, snorek@fel.cvut.cz \\
\hline Vice president & Mikuláš Alexík, alexik@frtk.fri.utc.sk \\
\hline Scientific Secr. & A. Kavička, Antonin.Kavicka@ upce.cz \\
\hline Repr. EuRoSIM & Miroslav Šnorek, snorek@fel.cvut.cz \\
\hline Edit. Board SNE & Mikuláš Alexík, alexik@frtk.fri.utc.sk \\
\hline Web EURoSIM & Petr Peringer, peringer@fit.vutbr.cz \\
\hline
\end{tabular}

\section{DBSS - Dutch Benelux Simulation Society}

The Dutch Benelux Simulation Society (DBSS) was founded in July 1986 in order to create an organisation of simulation professionals within the Dutch language area. DBSS has actively promoted creation of similar organisations in other language areas. DBSS is a member of EUROSIM and works in close cooperation with its members and with affiliated societies.

$\rightarrow$ www.DutchBSS.org

麦=la.w.heemink@its.tudelft.nl

$\triangle$ DBSS / A. W. Heemink

Delft University of Technology, ITS - twi,

Mekelweg 4, 2628 CD Delft, The Netherlands

\begin{tabular}{ll}
\hline DBSS Officers & \\
\hline President & A. Heemink, a.w.heemink@its.tudelft.nl \\
\hline Vice president & M. M ujica M ota, m.mujica.mota@ hva.nl \\
\hline Treasurer & M. M ujica M ota, m.mujica.mota@ hva.nl \\
\hline Secretary & P. M. Scala, p.m.scala@ hva.nl \\
\hline Repr. EuroSIM & M. M ujica M ota, m.mujica.mota@ hva.nl \\
\hline Edit. SNE/Web & M. M ujica M ota, m.mujica.mota@ hva.nl \\
\hline &
\end{tabular}

\section{FrancoSIM - Société Francophone de Simulation}

FRANCOSIM was founded in 1991 and aims to the promotion of simulation and research, in industry and academic fields.

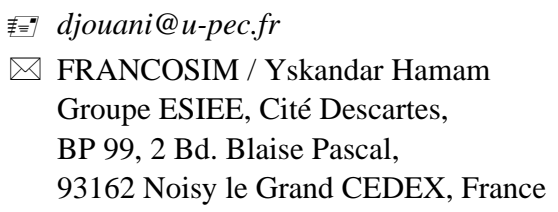

\begin{tabular}{ll}
\hline President & Karim Djouani, djouani@u-pec.fr \\
\hline Treasurer & François Rocaries, f.rocaries@ esiee.fr \\
\hline Repr. EuRoSım & Karim Djouani, djouani@u-pec.fr \\
\hline Edit. Board SNE & Karim Djouani, djouani@u-pec.fr \\
\hline
\end{tabular}




\section{HSS - Hungarian Simulation Society}

The Hungarian Member Society of EUROSIM was established in 1981 as an association promoting the exchange of information within the community of people involved in research, development, application and education of simulation in Hungary and also contributing to the enhancement of exchanging information between the Hungarian simulation community and the simulation communities abroad. HSS deals with the organization of lectures, exhibitions, demonstrations, and conferences.

$\rightarrow$ www.eurosim.info

麦三javor@eik.bme.hu

$\triangle$ HSS / András Jávor,

Budapest Univ. of Technology and Economics,

Sztoczek u. 4, 1111 Budapest, Hungary

\begin{tabular}{ll} 
HSS Officers & \\
\hline President & András Jávor, javor@ eik.bme.hu \\
\hline Vice president & Gábor Szúcs, szucs@itm.bme.hu \\
\hline Secretary & Ágnes Vigh, vigh@itm.bme.hu \\
\hline Repr. EuRoSım & András Jávor, javor@ eik.bme.hu \\
\hline Deputy & Gábor Szúcs, szucs@itm.bme.hu \\
\hline Edit. Board SNE & András Jávor, javor@ eik.bme.hu \\
\hline Web EuRoSım & Gábor Szúcs, szucs@itm.bme.hu \\
\hline
\end{tabular}

\section{ISCS - Italian Society for Computer} Simulation

The Italian Society for Computer Simulation (ISCS) is a scientific non-profit association of members from industry, university, education and several public and research institutions with common interest in all fields of computer simulation.

$\rightarrow$ www.eurosim.info

莑: Mario.savastano@uniina.it

$\triangle$ ISCS / Mario Savastano,

c/o CNR - IRSIP,

Via Claudio 21, 80125 Napoli, Italy

\begin{tabular}{ll}
\hline ISCS Officers & \\
\hline President & M. Savastano, mario.savastano@ unina.it \\
\hline Vice president & F. M aceri, Franco.M aceri@ uniroma2.it \\
\hline Repr. EuRoSIM & F. M aceri, Franco.M aceri@ uniroma2.it \\
\hline Secretary & $\begin{array}{l}\text { Paola Provenzano, } \\
\text { paola.provenzano@uniroma2.it }\end{array}$ \\
\hline Edit. Board SNE & M. Savastano, mario.savastano@ unina.it \\
\hline
\end{tabular}

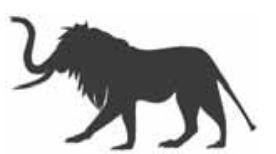

\section{LIOPHANT Simulation}

Liophant Simulation is a non-profit association born in order to be a trait-d'union among simulation developers and users; Liophant is devoted to promote and diffuse the simulation techniques and methodologies; the Association promotes exchange of students, sabbatical years, organization of International Conferences, courses and internships focused on $\mathrm{M} \& \mathrm{~S}$ applications.

$\rightarrow$ www.liophant.org

麦=7info@liophant.org

$\triangle$ LIOPHANT Simulation, c/o Agostino G. Bruzzone, DIME, University of Genoa, Savona Campus via Molinero 1, 17100 Savona (SV), Italy

\begin{tabular}{ll}
\hline \multicolumn{2}{l}{ LIOPHANT Officers } \\
\hline President & A.G. Bruzzone, agostino@ itim.unige.it \\
\hline Director & E. Bocca, enrico.bocca@liophant.org \\
\hline Secretary & A. Devoti, devoti.a@ iveco.com \\
\hline Treasurer & M arina M asseimassei@ itim.unige.it \\
\hline Repr. EuRoSIm & A.G. Bruzzone, agostino@ itim.unige.it \\
\hline Deputy & F. Longo, f.longo@ unical.it \\
\hline Edit. Board SNE & F. Longo, f.longo@ unical.it \\
\hline Web EURoSIM & F. Longo, f.longo@ unical.it \\
\hline
\end{tabular}

\section{LSS - Latvian Simulation Society}

The Latvian Simulation Society (LSS) has been founded in 1990 as the first professional simulation organisation in the field of Modelling and simulation in the postSoviet area. Its members represent the main simulation centres in Latvia, including both academic and industrial sectors.

$\rightarrow$ briedis.itl.rtu.lv/imb/

麦=! merkur@itl.rtu.lv

$\triangle$ LSS / Yuri Merkuryev, Dept. of Modelling and Simulation Riga Technical University Kalku street 1, Riga, LV-1658, LATVIA

\begin{tabular}{ll}
\hline LSS Officers & \\
\hline President & Yuri M erkuryev, merkur@ itl.rtu.Iv \\
\hline Secretary & Artis Teilans, Artis.Teilans@exigenservices.com \\
\hline Repr. EuROSIm & Yuri M erkuryev, merkur@ itl.rtu.Iv \\
\hline Deputy & Artis Teilans, Artis.Teilans@exigenservices.com \\
\hline Edit. Board SNE & Yuri M erkuryev, merkur@ itl.rtu.Iv \\
\hline Web EuROSIm & Vitaly Bolshakov, vitalijs.bolsakovs@ rtu.Iv \\
\hline
\end{tabular}




\section{KA-SIM Kosovo Simulation Society}

Kosova Association for Modeling and Simulation (KASIM, founded in 2009), is part of Kosova Association of Control, Automation and Systems Engineering (KACASE). KA-CASE was registered in 2006 as non Profit Organization and since 2009 is National Member of IFAC - International Federation of Automatic Control. KA-SIM joined EUROSIM as Observer Member in 2011. In 2016, KA-SIM became full member.

KA-SIM has about 50 members, and is organizing the international conference series International Conference in Business, Technology and Innovation, in November, in Durrhes, Albania, and IFAC Simulation Workshops in Pristina.

$\rightarrow$ www.ubt-uni.net/ka-case

拝”- ehajrizi@ubt-uni.net

$\triangle$ MOD\&SIM KA-CASE; Att. Dr. Edmond Hajrizi Univ. for Business and Technology (UBT)

Lagjja Kalabria p.n., 10000 Prishtina, Kosovo

\begin{tabular}{ll}
\hline KA-SIM Officers \\
\hline President & Edmond Hajrizi, ehajrizi@ ubt-uni.net \\
\hline Vice president & M uzafer Shala, info@ ka-sim.com \\
\hline Secretary & Lulzim Beqiri, info@ ka-sim.com \\
\hline Treasurer & Selman Berisha, info@ ka-sim.com \\
\hline Repr. EuRoSim & Edmond Hajrizi, ehajrizi@ ubt-uni.net \\
\hline Deputy & M uzafer Shala, info@ ka-sim.com \\
\hline Edit. Board SNE & Edmond Hajrizi, ehajrizi@ ubt-uni.net \\
\hline Web EuRoSIm & Betim Gashi, info@ ka-sim.com \\
\hline
\end{tabular}

\section{PSCS - Polish Society for Computer Simulation}

PSCS was founded in 1993 in Warsaw. PSCS is a scientific, non-profit association of members from universities, research institutes and industry in Poland with common interests in variety of methods of computer simulations and its applications. At present PSCS counts 257 members.

$\rightarrow$ www.eurosim.info (www.ptsk.man.bialystok.pl)

麦三leon@ibib.waw.pl

$\triangle$ PSCS / Leon Bobrowski, c/o IBIB PAN,

ul. Trojdena 4 (p.416), 02-109 Warszawa, Poland

\begin{tabular}{ll}
\hline PSCS Officers & \\
\hline President & Leon Bobrowski, leon@ibib.waw.pl \\
\hline Vice president & Tadeusz Nowicki, \\
& Tadeusz.Nowicki@ wat.edu.pl \\
\hline Treasurer & Z. Sosnowski, zenon@ii.pb.bialystok.pl \\
\hline Secretary & Zdzislaw Galkowski, \\
& Zdzislaw.Galkowski@ simr.pw.edu.pl \\
\hline Repr. EuroSım & Leon Bobrowski, leon@ibib.waw.pl \\
\hline Deputy & Tadeusz Nowicki, tadeusz.nowicki@ wat.edu.pl \\
\hline Edit. Board SNE & Zenon Sosnowski, z.sosnowski@pb.ed.pl \\
\hline Web EuRoSım & Magdalena Topczewska \\
& m.topczewska@ pb.edu.pl \\
\hline
\end{tabular}

\section{SIMS - Scandinavian Simulation Society}

SIMS is the Scandinavian Simulation Society with members from the four Nordic countries Denmark, Finland, Norway and Sweden. The SIMS history goes back to 1959 . SIMS practical matters are taken care of by the SIMS board consisting of two representatives from each Nordic country (Iceland one board member).

SIMS Structure. SIMS is organised as federation of regional societies. There are FinSim (Finnish Simulation Forum), DKSIM (Dansk Simuleringsforening) and NFA (Norsk Forening for Automatisering).

$\rightarrow$ www.scansims.org

麦=-esko.juuso@oulu.fi

$\triangle$ SIMS / Erik Dahlquist, School of Business, Society and Engineering, Department of Energy, Building and Environment, Mälardalen University, P.O.Box 883, 72123 Västerås, Sweden

\begin{tabular}{ll}
\hline SIM S Officers & \\
\hline President & Erik Dahlquist, erik.dahlquist@ mdh.se \\
\hline Vice president & Bernd Lie, lie@ hit.noe \\
\hline Treasurer & $\begin{array}{l}\text { Vadim Engelson, } \\
\text { vadim.engelson@ mathcore.com }\end{array}$ \\
\hline Repr. EuRoSIm & Erik Dahlquist, erik.dahlquist@ mdh.se \\
\hline Edit. Board SNE & Esko Juuso, esko.juuso@oulu.fi \\
\hline Web EuRoSım & $\begin{array}{l}\text { Vadim Engelson, } \\
\text { vadim.engelson@ mathcore.com }\end{array}$ \\
\hline
\end{tabular}




\section{SLOSIM - Slovenian Society for Simulation and Modelling}

SLOSIM - Slovenian Society for Simulation and Modelling was established in 1994 and became the full member of EUROSIM in 1996. Currently it has 90 members from both Slovenian universities, institutes, and industry. It promotes modelling and simulation approaches to problem solving in industrial as well as in academic environments by establishing communication and cooperation among corresponding teams.

$\rightarrow$ www.slosim.si

奉”slosim@fe.uni-lj.si

$\triangle$ SLOSIM / Vito Logar, Faculty of Electrical

Engineering, University of Ljubljana,

Tržaška 25, 1000 Ljubljana, Slovenia

\begin{tabular}{ll}
\hline \multicolumn{2}{l}{ SLOSIM Officers } \\
\hline President & Vito Logar, vito.logar@fe.uni-lj.si \\
\hline Vice president & Božidar Šarler, bozidar.sarler@ung.si \\
\hline Secretary & Aleš Belič, ales.belic@ sandoz.com \\
\hline Treasurer & Milan Simčič, milan.simcic@fe.uni-lj.si \\
\hline Repr. EuRoSım & B.Zupančič, borut.zupancic@fe.uni-lj.si \\
\hline Deputy & Vito Logar, vito.logar@fe.uni-lj.si \\
\hline Edit. Board SNE & B. Zupančič, borut.zupancic@fe.uni-lj.si \\
& Vito Logar, vito.logar@fe.uni-lj.si \\
& Blaž Rodič, blaz.rodic@fis.unm.si \\
\hline Web EuRoSım & Vito Logar, vito.logar@fe.uni-lj.si \\
\hline
\end{tabular}

\section{UKSIM - United Kingdom Simulation Society}

The UK Simulation Society is very active in organizing conferences, meetings and workshops. UKSim holds its annual conference in the March-April period. In recent years the conference has always been held at Emmanuel College, Cambridge. The Asia Modelling and Simulation Section (AMSS) of UKSim holds 4-5 conferences per year including the EMS (European Modelling Symposium), an event mainly aimed at young researchers, organized each year by UKSim in different European cities.

Membership of the UK Simulation Society is free to participants of any of our conferences and their coauthors.
UKSIM / Prof. David Al-Dabass

Computing \& Informatics,

Nottingham Trent University

Clifton lane, Nottingham, NG11 8NS

United Kingdom

\begin{tabular}{ll}
\hline UKSIM Officers & \\
\hline President & $\begin{array}{l}\text { David Al-Dabass, } \\
\text { david.al-dabass@ntu.ac.uk }\end{array}$ \\
\hline Secretary & A. Orsoni, A.Orsoni@ kingston.ac.uk \\
\hline Treasurer & A. Orsoni, A.Orsoni@ kingston.ac.uk \\
\hline Membership chair & G. Jenkins, glenn.l.jenkins@ smu.ac.uk \\
\hline Local/Venue chair & Richard Cant, richard.cant@ntu.ac.uk \\
\hline Repr. EuRoSıM & A. Orsoni, A.Orsoni@ kingston.ac.uk \\
\hline Deputy & G. Jenkins, glenn.l.jenkins@ smu.ac.uk \\
\hline Edit. Board SNE & A. Orsoni, A.Orsoni@ kingston.ac.uk \\
\hline &
\end{tabular}

\section{RNSS - Russian Simulation Society}

NSS - The Russian National Simulation Society (Национальное Общество Имитационного Моделирования - НОИМ) was officially registered in Russian Federation on February 11, 2011. In February 2012 NSS has been accepted as an observer member of EUROSIM, and in 2015 RNSS has become full member.

$\rightarrow$ www.simulation.su

麦”y yusupov@iias.spb.su

$\bowtie$ RNSS / R. M. Yusupov,

St. Petersburg Institute of Informatics and Automation RAS, 199178, St. Petersburg, 14th lin. V.O, 39

\begin{tabular}{ll}
\hline RNSS Officers & \\
\hline President & R. M. Yusupov, yusupov@iias.spb.su \\
\hline Chair Man. Board & A. Plotnikov, plotnikov@sstc.spb.ru \\
\hline Secretary & M. Dolmatov, dolmatov@simulation.su \\
\hline Repr. EuRoSIm & $\begin{array}{l}\text { R.M. Yusupov, yusupov@ iias.spb.su } \\
\text { Y. Senichenkov, } \\
\text { senyb@dcn.icc.spbstu.ru }\end{array}$ \\
\hline Deputy & B. Sokolov, sokol@iias.spb.su \\
\hline Edit. Board SNE & $\begin{array}{c}\text { Y. Senichenkov, } \\
\text { senyb@dcn.icc.spbstu.ru }\end{array}$ \\
\hline
\end{tabular}

$\rightarrow$ www.uksim.org.uk

莑=david.al-dabass@ntu.ac.uk 


\section{EUROSIM OBSERVER M EMBERS}

\section{ROM SIM - Romanian Modelling and Simulation Society}

ROMSIM has been founded in 1990 as a non-profit society, devoted to theoretical and applied aspects of modelling and simulation of systems. ROMSIM currently has about 100 members from Romania and Moldavia.

$\rightarrow$ www.eurosim.info (www.ici.ro/romsim)

拝三sflorin@ici.ro

$\triangle$ ROMSIM / Florin Hartescu,

National Institute for Research in Informatics, Averescu Av. 8 - 10, 71316 Bucharest, Romania

\begin{tabular}{lr}
\hline ROM SIM Officers \\
\hline $\begin{array}{l}\text { President } \\
\text { Vice president }\end{array}$ & $\begin{array}{r}\text { Florin Hartescu, flory@ici.ro } \\
\text { Marius Radulescu, } \\
\text { mradulescu.csmro@yahoo.com }\end{array}$ \\
\hline Repr. EUROSIM & $\begin{array}{r}\text { Marius Radulescu, } \\
\text { mradulescu.csmro@yahoo.com }\end{array}$ \\
\hline Deputy & Florin Hartescu, flory@ici.ro \\
\hline Edit. Board SNE & Constanta Zoe Radulescu, zoe@ ici.ro \\
\hline Web EuRoSIM & Florin Hartescu, flory@ici.ro \\
\hline
\end{tabular}

\section{MIMOS - Italian Modelling and Simulation Association}

MIMOS (Movimento Italiano Modellazione e Simulazione - Italian Modelling and Simulation Association) is the Italian association grouping companies, professionals, universities, and research institutions working in the field of modelling, simulation, virtual reality and 3D, with the aim of enhancing the culture of 'virtuality' in Italy, in every application area.

MIMOS became EUROSIM Observer Member in 2016 and is preparing application for full membership.

$\rightarrow w w w . m i m o s . i t$

麦”roma@mimos.it-info@mimos.it

$\triangle$ MIMOS - Movimento Italiano Modellazione e Simulazione; via Ugo Foscolo 4, 10126 Torino - via Laurentina 760, 00143 Roma

\begin{tabular}{ll}
\hline MIM 0S Officers & \\
\hline President & Paolo Proietti, roma@mimos.it \\
\hline Secretary & Davide Borra, segreteria@ mimos.it \\
\hline Treasurer & Davide Borra, segreteria@mimos.it \\
\hline Repr. EuRoSım & Paolo Proietti, roma@mimos.it \\
\hline Deputy & $\begin{array}{l}\text { Agostino Bruzzone, agosti- } \\
\text { no@ itim.unige.it }\end{array}$ \\
\hline Edit. Board SNE & Paolo Proietti, roma@ mimos.it \\
\hline & \multicolumn{1}{c}{ Last data update December 2016 }
\end{tabular}

\section{CANDidates}

\section{Albanian Simulation Society}

At the Department of Statistics and Applied Informatics, Faculty of Economy, University of Tirana, Prof. Dr. Kozeta Sevrani at present is setting up an Albanian Simulation Society. Kozeta Sevrani, professor of Computer Science and Management Information Systems, and head of the Department of Mathematics, Statistics and Applied Informatic, has attended a EUROSIM board meeting in Vienna and has presented simulation activities in Albania and the new simulation society.

The society - constitution and bylaws are being worked out - will be involved in different international and local simulation projects, and will be engaged in the organisation of the conference series ISTI - Information Systems and Technology. The society intends to become a EUROSIM Observer Member.

棒 kozeta.sevrani@unitir.edu.al

$\triangle$ Albanian Simulation Goup, attn. Kozeta Sevrani University of Tirana, Faculty of Economy rr. Elbasanit, Tirana 355 Albania

\begin{tabular}{|c|c|}
\hline \multicolumn{2}{|c|}{ Albanian Simulation Society- Officers (Planned) } \\
\hline President & $\begin{array}{l}\text { Kozeta Sevrani, } \\
\quad \text { kozeta.sevrani@unitir.edu.al }\end{array}$ \\
\hline Secretary & \\
\hline Treasurer & \\
\hline Repr. EUROSIM & $\begin{array}{l}\text { Kozeta Sevrani, } \\
\quad \text { kozeta.sevrani@unitir.edu.al }\end{array}$ \\
\hline Edit. Board SNE & $\begin{array}{l}\text { Albana Gorishti, } \\
\text { albana.gorishti@unitir.edu.al } \\
\text { Majlinda Godolja, } \\
\text { majlinda.godolja@fshn.edu.al }\end{array}$ \\
\hline
\end{tabular}




\section{ASIM - Buchreihen / ASIM Book Series}
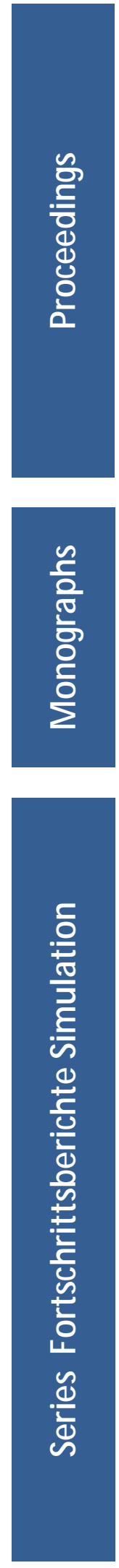

Simulation in Production and Logistics 2017 - 17. ASIM Fachtagung Simulation in Produktion und Logistik Sigrid Wenzel, Tim Peter (Hrsg.); kassel university press GmbH, Kassel, 2017; ISBN 978-3-7376-0192-4 (print), ISBN 978-3-7376-0193-1 (e-book); ASIM M itteilung AM 164.

Simulation in Production und Logistics 2015 - 16. ASIM-Fachtagung Simulation in Produktion und Logistik M. Raabe, U. Clausen (Hrsg.); ISBN 978-3-8396-0936-1, Stuttgart: Fraunhofer Verlag, 2015.

Simulation in Produktion und Logistik 2013: Entscheidungsunterstützung von der Planung bis zur Steuerung W. Dangelmaier, C. Laroque, A. Klaas (Hrsg.); ISBN 978-3-942647-35-9, HNI-Verlagsschriftenreihe, Heinz Nixdorf Institut, Paderborn, 2013.

Modellierung, Regelung und Simulation in Automotive und Prozessautomation - Proc. 5. ASIM-Workshop Wismar 2011. C. Deatcu, P. Dünow, T. Pawletta, S. Pawletta (eds.), ISBN 978-3-901608-36-0, ASIM /ARGESIM, Wien, 2011.

Simulation in Produktion und Logistik 2010: Integrationsaspekte der Simulation - Technik, Organisation und Personal. G. Zülch, P. Stock, (Hrsg.), ISBN 978-3-86644-558-1, KIT Scientific Publ. Karlsruhe, 2010.

Simulation und Optimierung in Produktion und Logistik - Praxisorientierter Leitfaden mit Fallbeispielen. L. März, W. Krug, O. Rose, G. Weigert , G. (Hrsg.); ISBN 978-3-642-14535-3, Springer, 2011.

Verifikation und Validierung für die Simulation in Produktion und Logistik - Vorgehensmodelle und Techniken. M. Rabe, S. Spieckermann, S. Wenzel (eds.); ISBN: 978-3-540-35281-5, Springer, Berlin, 2008.

Qualitätskriterien für die Simulation in Produktion und Logistik - Planung und Durchführung von Simulationsstudien. S. Wenzel, M. Weiß, S. Collisi - Böhmer, H. Pitsch, O. Rose (Hrsg.); ISBN: 978-3-540-35281-5, Springer, Berlin, 2008.

Patrick Einzinger: A Comparative Analysis of System Dynamics and Agent-Based M odelling for Health Care Reimbursement Systems. FBS 24; ISBN 978-3-901608-75-9, ASIM / ARGESIM Vienna, 2014; www.tuverlag.at - print-on-demand.

M artin Bruckner: Agentenbasierte Simulation von Personenströmen mit unterschiedlichen Charakteristiken. FBS 23; ISBN 978-3-901608-74-2 (ebook), ASIM/ARGESIM Vienna, 2014; www.tuverlag.at - print-on-demand

Stefan Emrich: Deployment of Mathematical Simulation Models for Space Management. FBS 22; ISBN 978-3-901608-73-5 (ebook), ASIM / ARGESIM Vienna, 2013; www.tuverlag.at - print-on-demand

Xenia Descovich: Lattice Boltzmann Modeling and Simulation of Incompressible Flows in Distensible Tubes for Applications in Hemodynamics. FBS 21; ISBN 978-3-901608-71-1 (ebook), ASIM /ARGESIM Vienna, 2012; www.tuverlag.at - print-on-demand

Florian Miksch: Mathematical Modeling for New Insights into Epidemics by Herd Immunity and Serotype Shift. FBS 20, ISBN 978-3-901608-70-4 (ebook), ASIM/ARGESIM Vienna, 2012; www.tuverlag.at - print-on-demand

Shabnam Tauböck: Integration of Agent Based Modelling in DEVS for Utilisation Analysis: The M oreSpace Project at TU Vienna. FBS 19; ISBN 978-3-901608-69-8 (ebook), ASIM/ARGESIM Vienna, 2012; www.tuverlag.at - print-on-demand

Christian Steinbrecher: Ein Beitrag zur prädiktiven Regelung verbrennungsmotorischer Prozesse. FBS 18; ISBN 978-3-901608-68-1 (print), 978-3-901608-72-8 (ebook), ASIM / ARGESIM Vienna, 2010.

Olaf Hagendorf: Simulation-based Parameter and Structure Optimisation of Discrete Event Systems FBS 17; ISBN 978-3-901608-67-4 (print), 978-3-901608-99-5 (ebook), ASIM/ARGESIM Vienna, 2010. 

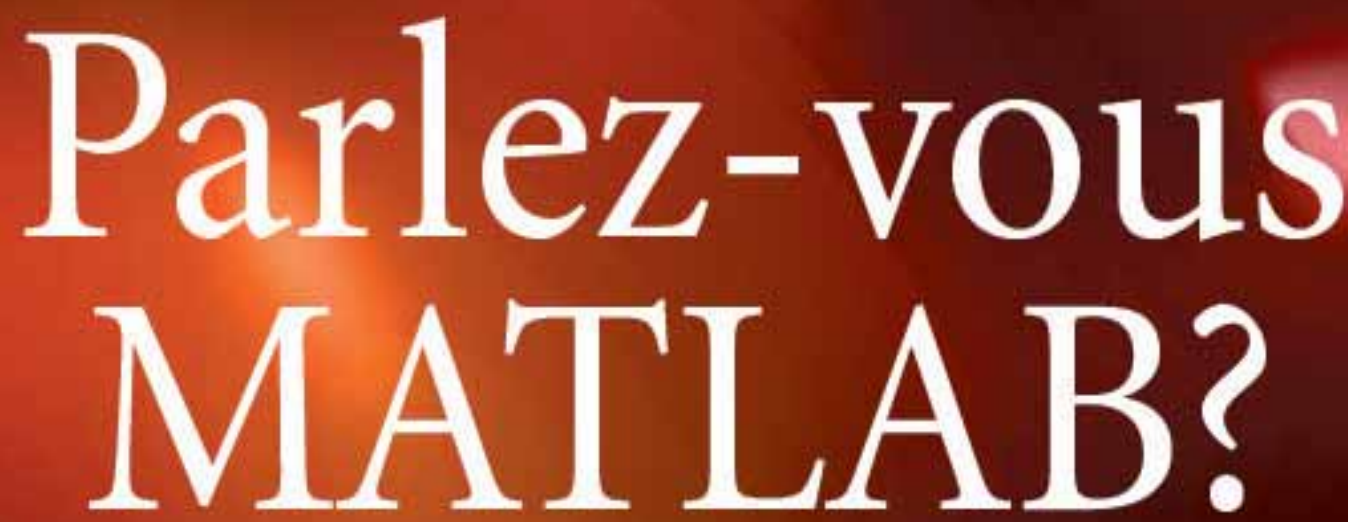

Ober eine Million Menschen wel twe it sprechen MATLAB. Ingenieure und Wissenschaftler in allen Bereichen - von der Luft- und Raumfahit tuber die Halbleiterindustrie bis zur Biotechnologie, Finanzdienstleistungen und Geo- und Mereswissenschuften - mutzen MATLAB, um ihre Ideen auszudrüdken. Sprechen Sie MATLAB?

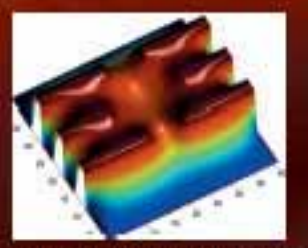

Malellierang cines elektrisches Potentials in cinem Qunumam Dot.

Dieses Reispiel finden Sie untern mwe mathworks dellic

The language of technical computing 